\title{
BEHAVIOURAL AND NEUROENDOCRINE IMPACTS OF ABUSE IN DATING RELATIONSHIPS
}

\author{
A thesis submitted to \\ the Faculty of Graduate Studies and Research \\ in Partial Fulfillment of the requirements for the degree \\ Doctor of Philosophy
}

by

Kerry A. Sudom

Department of Psychology

Carleton University

November 2007

C2007 Kerry Sudom 


$\begin{array}{ll}\begin{array}{l}\text { Library and } \\ \text { Archives Canada }\end{array} & \begin{array}{l}\text { Bibliothèque et } \\ \text { Archives Canada }\end{array} \\ \begin{array}{l}\text { Published Heritage } \\ \text { Branch }\end{array} & \begin{array}{l}\text { Direction du } \\ \text { Patrimoine de l'édition }\end{array} \\ \begin{array}{l}\text { 395 Wellington Street } \\ \text { Ottawa ON K1A 0N4 }\end{array} & \begin{array}{l}\text { 395, rue Wellington } \\ \text { Ottawa ON K1A ON4 }\end{array} \\ \text { Canada } & \begin{array}{l}\text { Canada } \\ \end{array}\end{array}$

Yourfile Votre référence

ISBN: 978-0-494-36794-0

Our file Notre référence

ISBN: 978-0-494-36794-0

NOTICE:

The author has granted a nonexclusive license allowing Library and Archives Canada to reproduce, publish, archive, preserve, conserve, communicate to the public by telecommunication or on the Internet, loan, distribute and sell theses worldwide, for commercial or noncommercial purposes, in microform, paper, electronic and/or any other formats.

The author retains copyright ownership and moral rights in this thesis. Neither the thesis nor substantial extracts from it may be printed or otherwise reproduced without the author's permission.
AVIS:

L'auteur a accordé une licence non exclusive permettant à la Bibliothèque et Archives Canada de reproduire, publier, archiver, sauvegarder, conserver, transmettre au public par télécommunication ou par l'Internet, prêter, distribuer et vendre des thèses partout dans le monde, à des fins commerciales ou autres, sur support microforme, papier, électronique et/ou autres formats.

L'auteur conserve la propriété du droit d'auteur et des droits moraux qui protège cette thèse. $\mathrm{Ni}$ la thèse ni des extraits substantiels de celle-ci ne doivent être imprimés ou autrement reproduits sans son autorisation.
In compliance with the Canadian

Privacy Act some supporting forms may have been removed from this thesis.

While these forms may be included in the document page count, their removal does not represent any loss of content from the thesis.
Conformément à la loi canadienne sur la protection de la vie privée, quelques formulaires secondaires ont été enlevés de cette thèse.

Bien que ces formulaires aient inclus dans la pagination, il n'y aura aucun contenu manquant.

\section{Canadä}




\begin{abstract}
Intimate partner abuse is associated with mental health disturbances, including depression and posttraumatic stress disorder (PTSD). In addition, abuse may be associated with certain psychological characteristics (e.g., appraisals, coping styles), and inadequate social support, that limit ability to respond effectively to relationship conflicts. As well, alterations in the hypothalamic-pituitary-adrenal (HPA) axis frequently accompany depressive and PTSD symptomatology, and may be evident in abused women. However, few studies have examined such factors, particularly in the earlier stages of intimate relationships. The present investigation was conducted to discern the impact of abuse (physical, sexual, and psychological) in dating relationships on symptoms of depression, PTSD, and neuroendocrine activity, and to assess whether appraisals, social support and coping mediated the effects of abuse. It was found that abuse was associated with increased symptoms of depression and PTSD, as well as variations in social support, appraisals, and coping. Furthermore, perceptions of low social support from one's partner, use of ineffective coping styles, and appraisals of lack of control over the outcomes of ambiguous situations within relationships, mediated the effects of abuse on well-being. There were no variations in neuroendocrine functioning in relation to abuse or symptoms of PTSD.
\end{abstract}




\section{Acknowledgments}

This dissertation would not have been possible without the help of a number of individuals. First and foremost, I am indebted to my advisor, Dr. Hymie Anisman. Without his continual support and guidance throughout the years, as well as his occasional friendly reminders to finish, this thesis would not have been attainable. Next, I would like to thank Dr. Kim Matheson for providing statistical expertise and many valuable recommendations. I am also grateful to have had the biochemical expertise of Dr. Jerzy Kulczycki.

Special thanks also go to my external examiner, Dr. Martine Flament, for providing such thorough and valuable feedback on my dissertation, as well as my other committee members, Dr. George Pollard, Dr. John Zelenski, and Dr. Janet Mantler, for their time and expertise.

Many fellow lab members were helpful in a variety of ways, from helping to run the studies, to providing encouragement and humour, including but not limited to Owen Kelly, Kelly Carroll, Alla Skomorovsky, and Renate Ysseldyk. The Carleton psychology department administrative staff was also very helpful. Finally, I would like to express appreciation for the support and understanding that I have had from family and friends along the way. 
Table of Contents

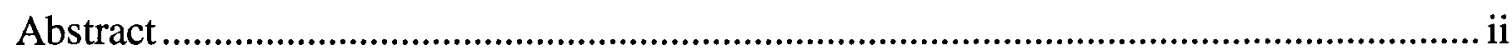

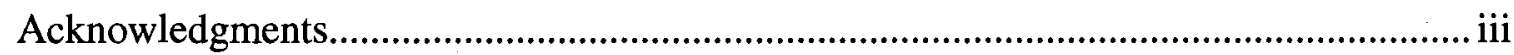

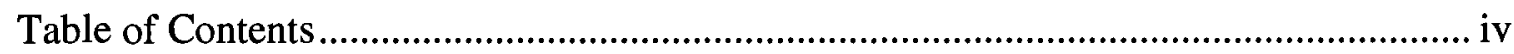

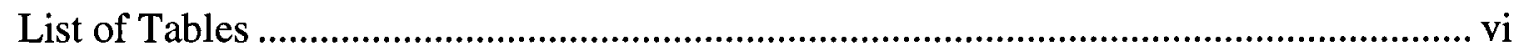

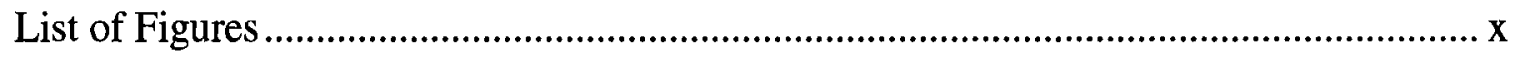

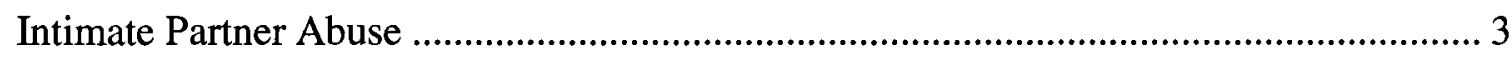

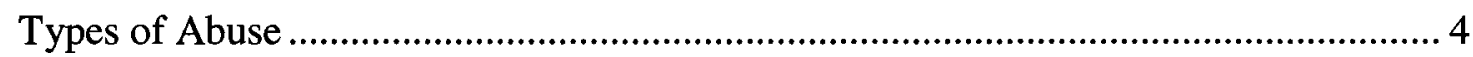

Abuse in Early Dating Relationships ........................................................................ 6

Abuse and Psychological Health: Effects of Abuse on Depression and PTSD .................. 8

Potential Mediators in the Relationship between Abuse and Pathology .......................... 10

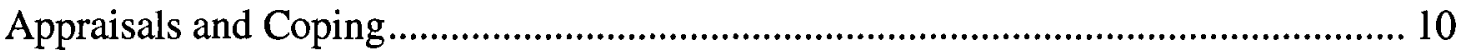

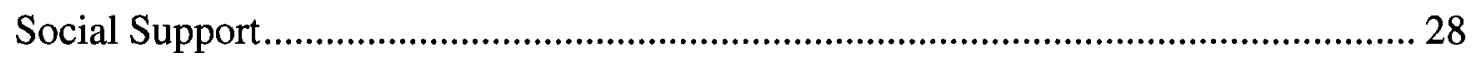

Neuroendocrine Variations associated with Abuse …........................................................ 33

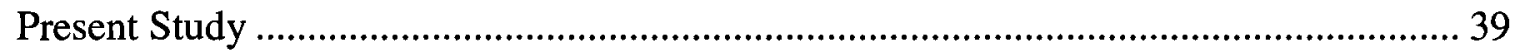

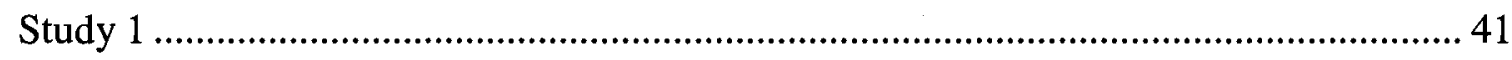

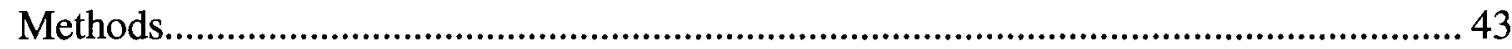

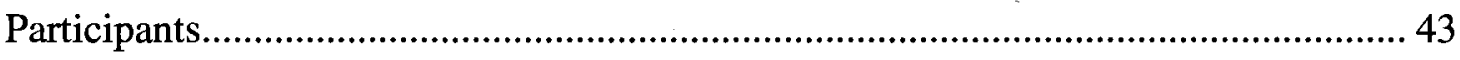

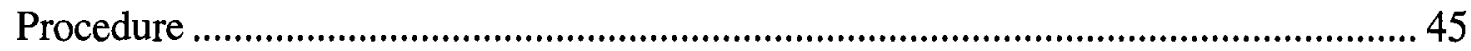

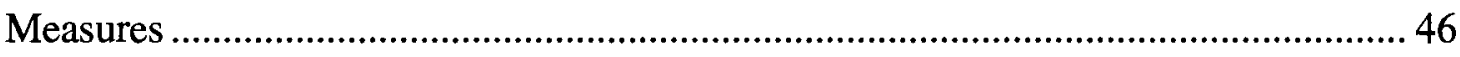

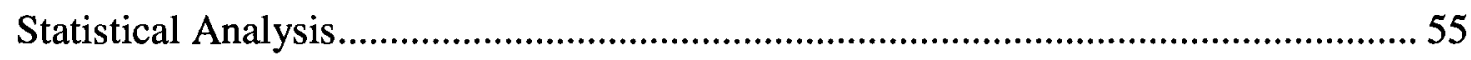

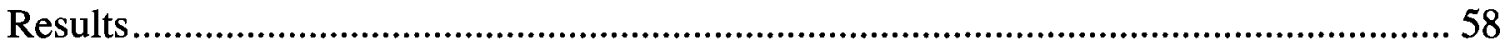

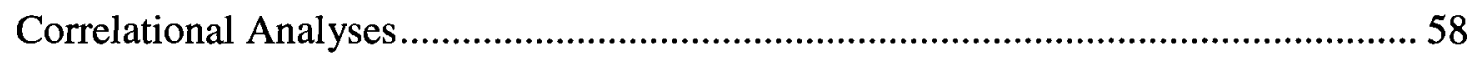


Role of Coping in Mediating the Relationship between Abuse and Well-Being ......... 59 Role of Social Support in Mediating the Relationship between Abuse and Well-Being 68

Neuroendocrine Response Elicited by a Challenge ............................................... 74

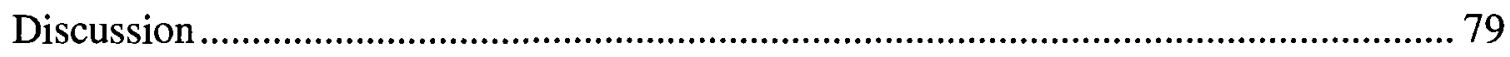

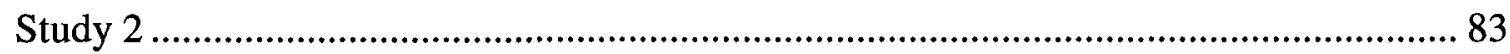

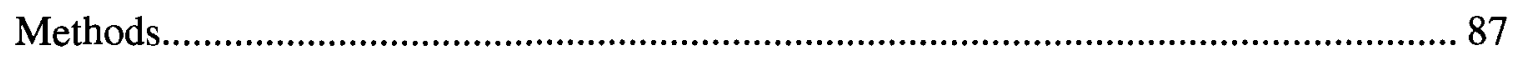

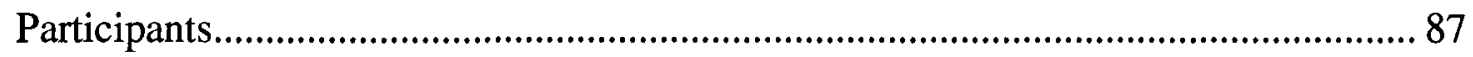

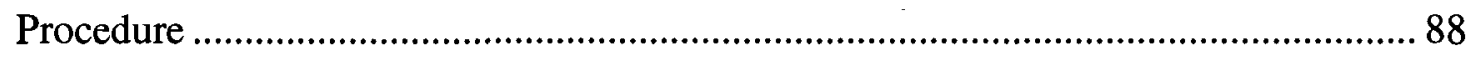

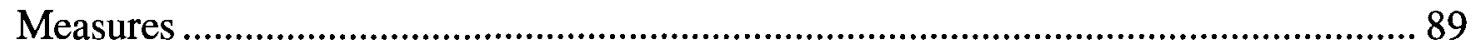

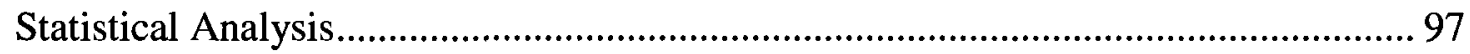

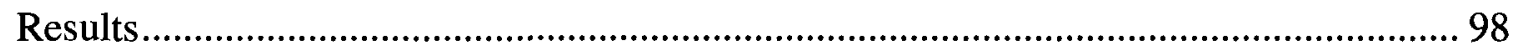

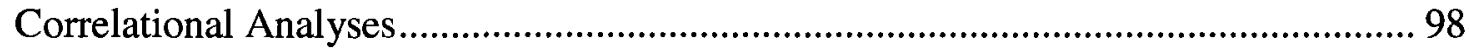

Role of Coping in Mediating the Relationship between Abuse and Well-Being ....... 101

Role of Appraisals in Mediating the Relationship between Abuse and Well-Being.. 110 Role of Appraisals in Mediating the Relationship between Abuse and Coping........ 120

Neuroendocrine Response Elicited by a Challenge ............................................ 123

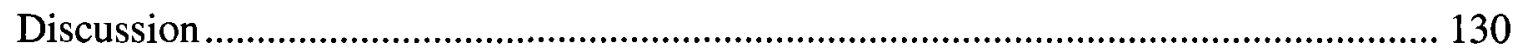

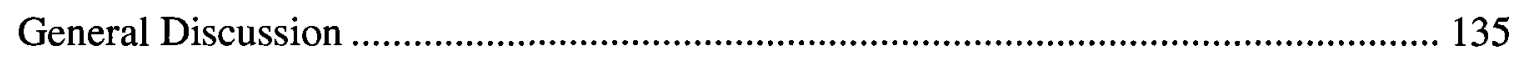

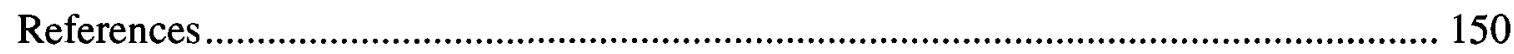

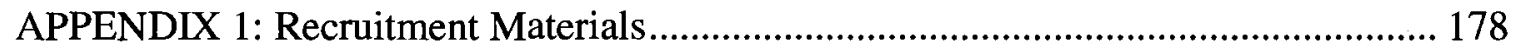

APPENDIX 2: Informed Consent and Debriefing............................................... 183

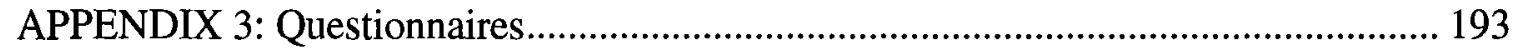




\section{List of Tables}

Table 1. Frequencies (and Percentages) of Previous Traumatic Experiences among

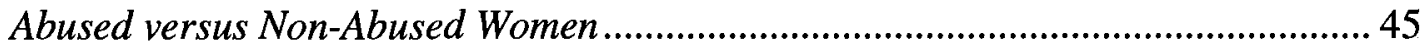

Table 2. Correlations Among the SCOPE Subscales................................................ 52

Table 3. Correlations Among the Dimensions of Coping ............................................. 53

Table 4. Regression Analysis (Pearson Correlations and Standardized Regression Coefficients) Assessing the Relations between Abuse and Well-Being ..................... 58

Table 5. Correlations between Coping and Social Support ........................................... 59

Table 6. Direct Effects of Abuse-Coping and Coping-Depression .................................. 62

Table 7. Direct and Indirect (through Coping) Effects of Abuse on Symptoms of Depression: Results of Bootstrap Analysis

Table 8. Bootstrap Confidence Intervals for the Total and Specific Mediating Effects of Coping on the Abuse-Depression Relationship 64

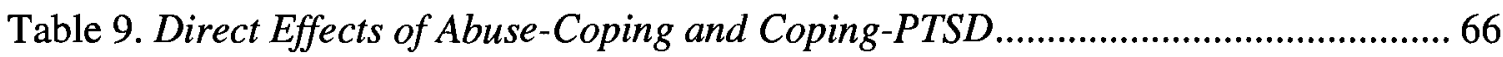

Table 10. Direct and Indirect (through Coping) Effects of Abuse on Symptoms of PTSD: Results of Bootstrap Analysis

Table 11. Bootstrap Confidence Intervals and Contrasts for the Total and Specific Mediating Effects of Coping on the Abuse-PTSD Relationship............................. 68

Table 12. Direct Effects of Abuse-Support and Support-Depression ............................. 70 Table 13. Direct and Indirect (through Social Support) Effects of Abuse on Symptoms of Depression: Results of Bootstrap Analysis 71

Table 14. Bootstrap Confidence Intervals for the Total and Specific Mediating Effects of Social Support on the Abuse-Depression Relationship 72 
Table 15. Direct Effects of Abuse - Social Support and Social Support - PTSD 73

Table 16. Cortisol Levels ( $\mu \mathrm{g} / \mathrm{dl})$ as a Function of Abuse Type 75

Table 17. Relationship of Abuse to Baseline Cortisol Levels 75

Table 18. Regression Coefficients Predicting Cortisol Ratios as a Function of

Psychological Abuse, PTSD Symptoms and Mild Challenge

Table 19. Regression Coefficients Predicting Cortisol Ratios as a Function of Sexual

Abuse, PTSD Symptoms and Mild Challenge 78

Table 20. Regression Coefficients Predicting Cortisol Ratios as a Function of Physical Abuse, PTSD Symptoms and Mild Challenge

Table 21. Frequencies (and Percentages) of Previous Traumatic Experiences among Abused versus Non-Abused Women 88

Table 22. Correlations among the SCOPE Subscales. 92

Table 23. Correlations among the Dimensions of Coping 93

Table 24. Correlations among the Dimensions of the ECS and the SCOPE. 94

Table 25. Regression Analysis (Pearson Correlations and Standardized Regression

Coefficients) Assessing the Relations between Abuse and Well-being .... 98

Table 26. Intercorrelations Among the Potential Mediating Variables 100

Table 27. Direct Effects of Abuse - Coping and Coping - Depression 103

Table 28. Direct and Indirect (through Coping) Effects of Abuse on Symptoms of Depression: Results of Bootstrap Analysis 104

Table 29. Bootstrap Confidence Intervals for the Total and Specific Mediating Effects of Coping on the Psychological Abuse-Depression Relationship 105

Table 30. Direct Effects of Abuse - Coping and Coping - PTSD 107 
Table 31. Direct and Indirect (through Coping) Effects of Abuse on Symptoms of PTSD:

Results of Bootstrap Analysis.

Table 32. Bootstrap Confidence Intervals and Contrasts for the Total and Specific

Mediating Effects of Coping on the Psychological Abuse-PTSD Relationship ...... 109

Table 33. Direct Effects of Abuse - Appraisals and Appraisals - Depression.

Table 34. Direct Effects of Abuse - Appraisals of Ambiguous Events and Appraisals Depression

Table 35. Direct and Indirect (through Appraisals) Effects of Abuse on Symptoms of Depression: Results of Bootstrap Analysis

Table 36. Bootstrap Confidence Intervals for the Total and Specific Indirect Effects of Appraisals of Ambiguous Events on the Psychological Abuse-Depression Relationship

Table 37. Direct Effects of Abuse - Appraisals and Appraisals - PTSD 116

Table 38. Direct Effects of Abuse - Appraisals of Ambiguous Event and Appraisals -

PTSD 118

Table 39. Direct and Indirect (through Appraisals) Effects of Abuse on Symptoms of PTSD: Results of Bootstrap Analysis.

Table 40. Bootstrap Confidence Intervals for the Total and Specific Indirect Effects of Appraisals of Ambiguous Events on the Psychological Abuse-PTSD Relationship 120

Table 41. Direct and Indirect (through Appraisals) Effects of Psychological Abuse on Coping: Results of Bootstrap Analysis

Table 42. Bootstrap Confidence Intervals for the Total and Specific Indirect Effects of Appraisals on the Abuse-Coping Relationship 
Table 43. Cortisol Levels ( $\mu \mathrm{g} / \mathrm{dl})$ as a Function of Abuse Type 123

Table 44. Relationship of Abuse to Baseline Cortisol Levels

Table 45. Regression Coefficients predicting 15 min Cortisol Ratio as a Function of Psychological Abuse, PTSD Symptoms and Stressor Challenge

Table 46. Regression Coefficients predicting 30 min Cortisol Ratio as a Function of Psychological Abuse, PTSD Symptoms and Stressor Challenge.

Table 47. Regression Coefficients predicting 15 min Cortisol Ratio as a Function of Sexual Abuse, PTSD Symptoms and Stressor Challenge. 127

Table 48. Regression Coefficients predicting 30 min Cortisol Ratio as a Function of Sexual Abuse, PTSD Symptoms and Stressor Challenge. 128

Table 49. Regression Coefficients predicting 15 min Cortisol Ratio as a Function of Physical Abuse, PTSD Symptoms and Stressor Challenge 129

Table 50. Regression Coefficients predicting 30 min Cortisol Ratio as a Function of Physical Abuse, PTSD Symptoms and Stressor Challenge 


\section{List of Figures}

Figure 1. Mediating Effects of Coping and Social Support on the Relationship between

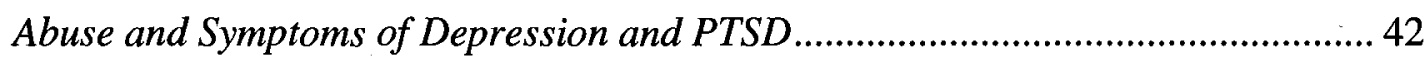

Figure 2. Mediating Effects of Coping and Appraisals on the Relationship Between Abuse and Symptoms of Depression and PTSD 
Behavioural and Neuroendocrine Impacts of Abuse in Dating Relationships Intimate partner abuse has been associated with mental and physical health problems, including depression, posttraumatic stress disorder (PTSD), anxiety, suicidal ideation, and suicide attempts (Bergman \& Brismar, 1991; Golding, 1999; Kemp, Green, Hovanitz, \& Rawlings, 1995; Pagelow; 1984). In addition to psychological disturbances, alterations of hypothalamic-pituitary-adrenal (HPA) activity frequently accompany depressive and PTSD symptomatology (e.g., Ehlert, Gaab, \& Heinrichs, 2001; Yehuda, Teicher, Trestman, Levengood, \& Siever, 1996), but it is uncertain to what extent altered HPA functioning might be associated with abuse, and whether the HPA alterations have beneficial or damaging effects. Although short-term elevations of neuroendocrine activity following an acute stressor may be adaptive, the excessive wear and tear (allostatic overload) stemming from a protracted stressor may result in adverse physiological effects that may contribute to pathology (McEwen, 2000). Given that abusive relationships may be prolonged, it is possible that continued corticoid elevations might contribute to behavioural disturbances.

Although various coping strategies may be employed to contend with their situation, these strategies do not appear to be consistently effective in buffering individuals against the psychological pathologies that arise. In particular, women in abusive relationships frequently utilize emotion-focused and avoidant strategies to deal with stressors, which may lead to decreased well-being (e.g., Clements \& Sawney, 2000). Such women may also display altered appraisals of events, and may not be able to recognize the gravity of their situation (Pipes \& LeBov-Keeler, 1997). It may be that owing to distorted appraisals of their situation, abused women are unable to invoke 
appropriate coping strategies. Furthermore, coping and appraisals operate in a reciprocal fashion, such that appraisals of threat or harm may influence the coping strategy adopted, and coping strategies may in turn lead to reinterpretation of events (Chang \& Strunk, 1999; Folkman \& Lazarus, 1985). Another factor that may influence the effects of abuse is social support. In this regard, several studies have demonstrated that support may buffer the negative effects of abuse on well-being (e.g., Coker, Smith, Thompson, McKeown, Bethea et al., 2002; Coker, Watkins, Smith, \& Brandt, 2003).

Although most research has involved abuse of a physical or sexual nature, it is evident that psychological maltreatment frequently occurs in the context of physical or sexual abuse, and may in fact be a key contributor to mental illness above and beyond the contribution of other forms of abuse (Katz \& Arias, 1999). In addition, although much of the available data concerning partner abuse have been derived from studies that focused on women who had either been severely battered or had endured abuse over extended periods, there is increasing evidence that abuse may begin early in an intimate relationship (Arias, Samios, \& O’Leary, 1987; Barnett, Martinez, \& Keyson, 1996; DeKeseredy \& Kelly, 1993; Kuffel \& Katz, 2002; Makepeace, 1981; Walker, 1984; White \& Koss, 1991). The study of relationships at such an early stage is thus critical in order to determine the predictors and precursors of abuse and for the development of effective intervention strategies.

The present investigation was conducted to discern the impact of abuse (physical, sexual, and psychological) in dating relationships on the presence of symptoms of depression, PTSD and altered neuroendocrine activity in response to a stressor. As well, 
it was of interest to assess whether appraisals, social support and coping processes mediated the influence of abuse on mental health symptoms.

\section{Intimate Partner Abuse}

According to the Centers for Disease Control and Prevention (CDC), intimate partner abuse comprises physical and sexual violence, threats of violence, and emotional or psychological maltreatment initiated by a romantic partner (Saltzman, Green, Marks, $\&$ Thacker, 2000). The prevalence of intimate partner violence has been found to vary between $1.8 \%$ (in a national study) and $14.4 \%$ (among emergency room patients), while lifetime prevalence rates were estimated at as high as $53.6 \%$ when emotional or psychological abuse was considered (Coker, Smith, Bethea, King, \& McKeown, 2000; Vest, Catlin, Chen, \& Brownson, 2002).

Not surprisingly, women experiencing abuse have a high incidence of mental and physical health problems, including depression, fatigue, listlessness, alcohol and drug abuse, disruption of interpersonal relationships, somatic complaints (e.g., chronic headaches) and suicide attempts, compared to those who had not experienced abuse (Astin, Lawrence, \& Foy, 1993; Coker et al., 2000; Golding, 1999; Goodman, Koss, \& Russo, 1993). As well, a high prevalence of PTSD, a common response to trauma that includes symptoms such as re-experiencing the traumatic event, numbing, and hypervigilance, has been found among battered women (Kemp et al., 1995). Battered women experience strikingly high levels of hospital care compared to non-abused women, particularly for suicide attempts, psychiatric and gynecological disorders, and traumatic injuries (Bergman \& Brismar, 1991). As well, therapeutic drug use (antidepressants, tranquilizers, and pain medications), as well as recreational drug use, 
were elevated among women experiencing dating violence relative to nonabused women (Slashinski, Coker, \& Davis, 2003). Finally, abuse may also lead to altered functioning of the hypothalamic-pituitary-adrenal (HPA) axis. Although it is generally assumed that increased HPA activity may be adaptive in responding to short-term stressors, the wearand-tear associated with a protracted stressor (such as partner abuse) may have adverse psychological and physiological implications (Lupien, Nair, Briere, Maheu, Tu et al., 1999; McEwen, 2000).

\section{Types of Abuse}

Although the majority of research has concerned the impact of physical or sexual assault, psychological maltreatment has been examined by some researchers as a variable distinct from these other forms of abuse (e.g., O'Leary, 2001; Pico-Alfonso, 2005). The study of psychological abuse as a separate entity has been problematic, in part, due to a lack of consensus on definitions of what constitutes abuse, and at what point normal behaviour during relationship conflicts may be considered maltreatment. As well, psychological aggression frequently occurs in the context of other forms of abuse, making it difficult to disentangle the relative effects of different types of maltreatment. Furthermore, it has been suggested that the paucity of research regarding this form of maltreatment may be due, to some extent, to the necessity of responding to the acute ramifications of physical abuse, coupled with the view that the consequences of psychological maltreatment will be fewer and less severe than those of physical assault (Arias \& Pape, 1999). A focus on physical aggression, however, is dangerous in that it fails to consider the importance of the psychological or emotional maltreatment that frequently leads up to physical violence, and it positions this type of violence as the most 
important to be studied, minimizing the harm that other forms of abuse may cause (Olson, Fine, \& Lloyd, 2005). In fact, psychological victimization has been considered by some researchers to comprise non-serious negative interactions in relationships, or as simply a prelude to physical or sexual violence, rather than abuse in and of itself (DeKeseredy \& Schwartz, 1998b).

Generally, psychological abuse is considered to involve aversive behaviours enacted by an individual's partner (e.g., hostile communication, lack of affection, social isolation, social humiliation, economic deprivation, jealousy/possessiveness) such that the victim feels threatened that her capacity to carry out normal work- and family/social-related functions, as well as to have normal physical and mental health, may be disrupted (Coker et al., 2000; Hoffman, 1984; Katz \& Arias, 1999; Straight, Harper, \& Arias, 2003). Thus, inherent in this definition is the need to distinguish occasional aversive behaviour from protracted abusive behaviour, which may have adverse long-term effects on the victim.

To be sure, it is recognized that various forms of abuse frequently co-occur. For example, approximately $45 \%$ of physically assaulted women also reported sexual abuse in their relationships (Campbell \& Soeken, 1999). As well, $88 \%$ of a sample of women in relationships who had experienced physical or sexual abuse also reported concurrent psychological maltreatment (Coker et al., 2000). However, it is important to recognize the severity of the effects of psychological abuse in addition to those of sexual and physical abuse. Importantly, psychological abuse may be a key contributor to mental illness above and beyond the contribution of other forms of maltreatment (Katz \& Arias, 1999; Pico-Alfonso, 2005; Taft, Murphy, King, Dedeyn, \& Musser, 2005). In fact, women in physically abusive relationships frequently report the concurrent psychological 
abuse as being more detrimental to their well-being (e.g., inducing feelings of passivity, social isolation, and shame) (Follingstad, Rutledge, Berg, Hause, \& Polek, 1990). Moreover, once the effects of verbal assault were controlled for, physically abused women were similar to non-abused women in depressive symptoms and self-esteem (Orava, McLeod, \& Sharpe, 1996). Additionally, when the effects of physical abuse were controlled, psychological abuse remained significantly correlated with psychological adjustment, suggesting that it is, at least, as detrimental to well-being as physical abuse (Arias \& Pape, 1999).

Abuse in Early Dating Relationships

The majority of research on intimate partner violence has involved individuals from battered women's shelters or hospital outpatient units, who had likely been experiencing abuse for a protracted period of time. It has been suggested that such helpseeking populations may not be representative of all victims of intimate partner violence. Differences may exist, for example, in the frequency or severity of violence experienced, or in access to resources and social support (Waldrop \& Resick, 2004). Importantly, abuse may begin early in an intimate relationship, increasing progressively, albeit intermittently, thereafter (Arias et al., 1987; DeKeseredy \& Kelly, 1993; Kuffel \& Katz, 2002; Makepeace, 1981; White \& Koss, 1991). Indeed, date abuse among university women is not infrequent, reaching $20 \%$ in the case of physical abuse and $80 \%$ when psychological abuse is considered (depending on the criteria used to define abuse) (Banyard, Arnold \& Smith, 2000; Makepeace, 1981). In addition, approximately $30 \%$ of adolescent women reported an experience of rape/attempted rape or verbal sexual coercion (Rickert, Wiemann, Vaughan, \& White, 2004). Some reports have indicated that 
younger women are at even greater risk for experiencing such violence than older women. For example, approximately $5 \%$ of women under the age of 25 reported at least one incident of violence within the past 12 months in their current relationship, compared to $1 \%$ of women 45 and older (Statistics Canada, 2000, 2005). Other studies have confirmed the relatively high prevalence of abuse within intimate relationships among younger women, indicating that abuse generally peaks between 20 and 25 years of age (O’Leary, 1999; Vest et al., 2002).

Abuse in young adulthood may be particularly damaging to mental and physical well-being, often being associated with excessive dieting behaviours, illicit drug use, alcohol abuse, involvement in risky sexual behaviour, suicidal ideation and attempts, low self-esteem, lower health-related quality of life, and depression (Ackard, NeumarkSztainer, \& Hannan, 2003; Coker, McKeown, Sanderson, Davis, Valois et al., 2000; Howard \& Wang, 2003). As well, half of adolescents in abusive relationships reported remaining in such relationships out of fear of being physically hurt if they attempted to leave (Ackard et al., 2003).

In addition to being potentially more frequent, abuse experienced during this period may be more severe than abuse later in life. For example, it was reported that battered women in their mid-twenties to thirties utilized somatic and psychiatric hospital care services at a greater rate than older women (Bergman \& Brismar, 1991). As well, although PTSD symptoms following abuse may occur at any point during an individual's lifetime, the likelihood of developing PTSD as a result of abuse was highest among women in their mid-20s (Yoshihama \& Horrocks, 2003). Evidently, younger women in the earlier stages of dating relationships may be at a greater risk for mental health 
problems related to experiences of abuse. Thus, younger women ought to be a primary target for the implementation of educational and intervention strategies, since negative patterns of dealing with conflicts and disagreements may begin to form in early stages of relationships. Indeed, early intervention strategies may be more effective than those occurring later, after violence has escalated, since negative behaviours and patterns of interaction may be more amenable to change relatively early in a relationship (Saltzman et al., 2000).

Abuse and Psychological Health: Effects of Abuse on Depression and PTSD Women experiencing abuse are at risk for a number of mental health disorders, particularly depression and PTSD (Golding, 1999). Although the experience of abuse within an intimate relationship may lead to symptoms of psychological distress, there is also evidence to indicate that the resultant symptoms may contribute to the maintenance of abuse. Indeed, the cognitive and motivational disturbances associated with depressive illness may be associated with a decreased likelihood of seeking treatment or alternatives to the relationship, as well as reduced vigilance or awareness of indicators of maltreatment (Clements \& Sawhney, 2000). In effect, features of depression or posttraumatic stress arising as a result of abuse may limit women's ability to respond effectively to relationship conflicts.

PTSD is an anxiety disorder characterized by intrusive thoughts about the stressful experience, avoidance of situations that may remind the individual of the event, emotional numbing, and hyperarousal in response to reminder stimuli (American Psychiatric Association, 1994). Although much of the previous research on PTSD has been conducted with samples of combat veterans and accident victims, symptoms can 
develop in response to a variety of stressful experiences, including aggression by an intimate partner. The prevalence of PTSD among abused women varies substantially from study to study (ranging from 31-84\%), possibly owing to factors such as the instruments and analytic techniques being used, sampling and recruitment strategies (e.g., population-based versus clinical samples; self-report versus clinical interview), and amount of time since the abuse (Golding, 1999). As well, it is possible that the nature of the abuse experienced may be relevant to the development of PTSD symptoms. Indeed, there is reason to believe that PTSD may be particularly likely to occur after the experience of emotional or verbal maltreatment, since psychological abuse was found to be a unique, significant predictor of PTSD symptoms, above and beyond the effects of physical abuse (Street \& Arias, 2001). Furthermore, the extent and severity of partner violence experienced is significantly associated with the degree of PTSD symptomatology (Houskamp \& Foy, 1991; Woods \& Isenberg, 2001).

In addition to PTSD appearing alone, depressive illness has frequently been reported to be comorbid with symptoms of PTSD among combat veterans (e.g., Mellman, Randolph, Brawman-Mintzer, Flores, \& Milanes, 1992), and this is true of victimized women as well (Marais, de Villiers, Moller, \& Stein, 1999; Resnick, Kilpatrick, Best \& Kramer, 1992; Stein \& Kennedy, 2001). In fact, it has been suggested that major depression among abused women rarely occurs in isolation, but is most common in the aftermath of abuse-related PTSD (Stein \& Kennedy, 2001). Although frequently occurring together, PTSD and depression appear to represent distinct but related disorders. Among couples seeking treatment for serious marital difficulties, although both depression and PTSD were related to the frequency of severe husband-to-wife 
aggression, PTSD symptoms were also uniquely related to the frequency of the partner's dominance/isolation behaviours and fear of the spouse, while depression was predicted by marital discord (Cascardi, O'Leary, \& Schlee, 1999). Thus, the disorders may be predicted by different factors, with PTSD occurring in the context of fear and intimidation, and depression resulting from relationship problems.

Although it is clear that the experience of abuse within an intimate relationship is associated with an increased incidence of mental health problems, certain variables may serve to mediate this association. That is, the effects of abuse on well-being may in part depend upon intervening variables. In particular, social support and coping styles, as well as cognitive or emotional factors such as characteristic appraisals of life events, may affect the appearance of symptoms of depression and PTSD among women in abusive dating relationships. These factors are discussed in turn in the sections that follow.

Potential Mediators in the Relationship between Abuse and Pathology

\section{Appraisals and Coping}

Coping and appraisals are intricately related and influence one another during an experience with a stressor. When a stressor is encountered, an individual appraises it in terms of its relevance to his or her well-being, as well as in relation to the coping strategies available to deal with the stressor. Most events are not considered inherently stressful, but instead depend upon the subjective judgement of the situation as being threatening or harmful (Zakowski, Hall, Klein, \& Baum, 2001). According to cognitiverelational theory, appraisal is a process of evaluating and categorizing the personal implications of events (Peacock \& Wong, 1990) and comprises two phases, primary and secondary appraisal (Folkman \& Lazarus, 1980). During the primary appraisal 
component, individuals assess what is at risk (Folkman \& Lazarus, 1980). A situation may be appraised as irrelevant, beneficial, or stressful (Peacock \& Wong, 1990). Whereas appraisals of harm or loss occur as a result of past events (i.e., damage that has already occurred), threat (anticipated harm or loss) and challenge (anticipated opportunity for mastery) appraisals ordinarily occur in response to anticipated events (Folkman \& Lazarus, 1980; Peacock \& Wong, 1990). As well, centrality (i.e., the importance of the situation to the individual; how much the individual has at stake in the situation) plays an important role in the appraisal process (Peacock \& Wong, 1990).

The secondary appraisal component involves the assessment of what can be done in a given situation. That is, the individual assesses whether he or she has the coping resources to contend with the stressor (Folkman \& Lazarus, 1980). Coping refers to the thoughts and behaviours utilized in situations that are perceived as both personally significant and as challenging the individual's resources for dealing with the situation (Folkman \& Lazarus, 1980; Folkman \& Moskowitz, 2004). After coping with a stressor, an individual may reappraise the situation; thus, stress and coping may be considered a continual process of appraisals and reappraisals (Zakowski et al., 2001). Coping has generally been categorized as either problem-focused (i.e., aimed at problem solving or addressing the source of the stressor) or emotion-focused (i.e., concerned with attenuating the negative emotions induced by the stressor; e.g., Folkman \& Lazarus, 1980). Lazarus and Launier (1978) suggested that the type of primary appraisal (i.e., threat, harm, loss, or challenge) is related to the specific type of coping strategy that is ultimately used. For example, it was found that when a situation was appraised as threatening, individuals tended to use emotion-focused coping strategies (e.g., wishful thinking), whereas both 
problem- and emotion-focused strategies were used in response to appraisals of challenge (McCrae, 1984). As well, perceiving greater personal stake in the outcome of a stressful event was associated with the use of more disengaged coping strategies, which deflect focus away from the source of the stressor and the reactions to it (Chang \& Strunk, 1999). Within the broad problem- and emotion-focused coping categorizations are a variety of specific strategies that can be used to deal with stressors. For example, emotion-focused coping encompasses strategies such as emotional expression, emotional containment, passive resignation, rumination, self-blame, and other-blame (Matheson \& Anisman, 2003). Likewise, these investigators suggested that cognitive restructuring may be considered within the problem-solving category. In addition, Endler and Parker (1990) proposed a third dimension of coping, namely that of avoidance. Since problem-focused, emotion-focused, and avoidant coping show low, non-significant correlations with each other, these classes may represent three distinct categories of dealing with adverse events. According to Endler and Parker (1990), problem-focused coping refers to a task orientation, whereas emotion-focused coping indicates a person-oriented approach. A person-oriented approach to coping involves strategies such as emotional responsiveness, preoccupation with the self, and fantasizing (i.e., wishful thinking). Avoidance may entail the use of either person-focused (e.g., seeking support) or task-focused strategies (e.g., engaging in another task to avoid the stressor). Another commonly used categorization of coping strategies that has been posited is approach versus avoidance, or active versus passive coping (Billings \& Moos, 1981; Suls \& Fletcher, 1985). Although the conceptualization of coping offered by Folkman and Lazarus (1980) emphasizes the function of coping (i.e., managing the problem versus managing the emotions associated 
with the problem), the latter approach concerns the focus of coping (i.e., the type of action - approach or avoidance - used to deal with the stressor) (Blalock \& Joiner, 2000). In considering coping processes, it is important to distinguish between coping styles and coping strategies. Whereas coping styles are considered characteristic ways of dealing with stressful situations, specific coping strategies endorsed may differ from situation to situation (Heszen-Niejodek, 1997). From the perspective of coping styles, individuals can be classified according to the way they generally react in stressful situations (e.g., Miller, 1992). Although coping styles may be considered as being akin to traits, which are relatively stable across situations, it is recognized that the specific strategies used may vary depending on the particular demands of the situation (Zakowski et al., 2001). In fact, flexibility and variability may reflect a component of coping style, such that individuals may have in their repertoire a variety of coping strategies, and the specific strategy used is dependent upon the context. Indeed, it has been suggested that flexibility in the use of strategies is key to coping successfully with stressful events (Heszen-Niejodek, 1997).

Although it is recognized that individuals may actively select particular coping strategies depending on the situation, the range of coping behaviours that each individual has at his or her disposal is limited, and it is likely that individuals have preferred strategies that they tend to endorse across situations. Of course, they may use different strategies from within their repertoire as the situation demands (Endler \& Parker, 1990). Other investigators (e.g., Lazarus \& Folkman, 1984) have tended to downplay the concept of characteristic ways of coping, in favour of emphasizing the importance of contextual influences on coping strategies endorsed, since the effectiveness of a given 
strategy may vary across situations (Folkman \& Moskowitz, 2004). These positions are, of course, not mutually exclusive. It is certainly possible that individuals are predisposed toward certain strategies based on their characteristic style of coping, but the specific strategies endorsed may depend upon aspects of the stressor (Heszen-Niejodek, 1997). In this regard, Gail and Evans (2001) emphasized the multidimensional nature of coping, asserting that the assessment of particular aspects of a situation (e.g., need for information, familiarity, and need to accept) may be fundamental in determining the perceived range of coping strategies available to deal with the situation.

Although a variety of coping strategies may be used in any given situation, it was suggested that perceived controllability over the stressor is an important factor in predicting which strategy will be used (Folkman \& Lazarus, 1980). In the main, problemfocused coping tends to prevail in stressful situations that are appraised as being controllable, while emotion-focused strategies are used in situations perceived as uncontrollable (Bijttebier, Vertommen, \& Vander Steene, 2001). For example, workrelated stressful encounters are associated with increased use of problem-solving, while emotion-focused strategies tend to be used in the context of health-related stressors, which may be less amenable to individual control (Folkman \& Lazarus, 1980). However, it is important to note that different coping strategies do not necessarily occur in isolation of one another, and indeed may be used simultaneously to varying degrees during a stressful encounter (Folkman \& Lazarus, 1980). In the sections that follow, the relationships among appraisals, coping, and well-being are discussed, as well as previous research on alterations in appraisals and coping processes among women who have experienced intimate partner violence. 
Interactions between appraisals, coping and well-being. Appraisals, coping, and psychological well-being are interdependent. Certain coping methods have consistently been associated with psychological disturbances. For example, dysphoria among university students was associated with the use of disengaged (i.e., avoidant), but not engaged (i.e., active) coping strategies when dealing with exam stress (Chang \& Strunk, 1999). Although appraisals may influence the type of coping strategy used (e.g., Chang \& Strunk, 1999), coping and appraisals likely operate in a dynamic, reciprocal fashion such that the outcomes of engaging in a coping activity may in turn modify one's appraisals of a situation (Folkman \& Lazarus, 1985). Although depressive symptoms (i.e., dysphoria) were directly associated with physical and psychological adjustment (e.g., life satisfaction, physical health symptoms), the association between depressive symptoms and adjustment was also found to be mediated by appraisals and coping (Chang \& Strunk, 1999). That is, in addition to its direct effects, dysphoria also had an indirect effect on well-being due to its influence on primary appraisal and coping processes. It has been suggested that the relationship between coping and distress (e.g., depressive symptoms) is reciprocal, such that distress leads to greater use of ineffective coping strategies, and the consequences of using these strategies leads to higher levels of distress (Blalock \& Joiner, 2000).

Inasmuch as emotion-focused coping, and particularly rumination (NolenHoeksema, 2000), emotional containment (Ravindran et al., 2002), and self- and otherblame (Matheson \& Anisman, 2003), is associated with behavioural disturbances such as depression, this form of coping has generally been considered to be maladaptive. In this regard, certain strategies such as rumination, or persistently thinking about and focusing 
on symptoms and meanings of distress, may induce or prolong depressive episodes via their negative effects on problem solving, perceptions of the self, hopelessness about the future, and perceived social support (Nolen-Hoeksema, 2000). Cognitive avoidance, which involves denying or minimizing the gravity or consequences of a stressful event and passively accepting that nothing can be done to alter a situation, was also associated with symptoms of depression and anxiety among women (Blalock \& Joiner, 2000).

Despite the commonly held view emphasizing the negative effects of emotionfocused coping strategies, such strategies may, in some situations, be beneficial. Researchers have begun to consider the positive qualities of emotions and the expression of emotions, emphasizing the adaptive nature of a coping style categorized as emotional approach. In contrast to other conceptualizations of emotion-focused coping, which contain elements of disengagement or denial (e.g., avoidance, wishful thinking) and involve elements of distress or self-deprecation, emotional approach coping involves the use of emotions to deal actively with a stressor, and comprises both emotional processing (i.e., actively engaging in exploring the meanings of, and coming to an understanding of, one's emotions) and expression (e.g., social support seeking or journal writing; Austenfeld \& Stanton, 1994; Stanton Danoff-Burg, Cameron, \& Ellis, 2004; Stanton, Kirk, Cameron, \& Danoff-Burg, 2000b).

Several lines of evidence point to the efficacy of emotion-focused styles of coping in responding to stressors. Emotional approach coping was associated with lower anxiety and depressive symptoms, as well as greater life satisfaction and self-esteem, among women (Stanton et al., 1994; Stanton, Danoff-Burg, Cameron, Bishop, \& Collins, 2000a). Moreover, emotional expression and processing were related to the use of other 
constructive coping strategies such as problem solving, and uncorrelated with avoidance coping (Stanton et al., 2000b), and emotional processing was negatively correlated with the use of passive coping (e.g., mental disengagement, wishful thinking) in a sample of individuals with chronic myofascial pain (Smith, Lumley, \& Longo, 2002). However, the efficacy of emotional approach for mitigating psychological distress may depend upon contextual characteristics and attributes of the stressor. For example, expression of emotions was associated with improved quality of life and decreased distress among individuals coping with a terminal illness, if they perceived their social environment as receptive to such expression (Stanton et al., 2000a). As well, emotional processing was most effective in mitigating symptoms of depression when used in interpersonal, but not achievement-oriented contexts, and in stressful situations perceived as uncontrollable (Stanton et al., 2004). Furthermore, although such studies have pointed to the positive effects of emotional approach, such a coping strategy may be efficacious only when performed intentionally. In this regard, involuntary emotional processing (e.g., constantly thinking of the emotions resulting from a stressful situation, while at the same time trying to engage in problem solving) may lead to rumination, while the inadvertent expression of emotion (e.g., being unable to control one's feelings of anger) may produce negative emotional outbursts (Compas, Connor, Osowiecki, \& Welch, 1997; Stanton et al., $2000 \mathrm{~b}$ ). Further, among individuals coping with a terminal illness, the endorsement of emotional processing strategies (aimed at examining and/or analyzing one's feelings to get a thorough understanding of them), although beneficial in the short term, led to increased rumination and distress over time (Stanton et al., 2000a). In contrast to the positive effects of emotional expression, attempts at suppression of emotional thoughts 
may actually lead to an increase in the intrusion of those thoughts, akin to rumination, as well as increased anxiety (Roemer \& Borkovec, 1994; Stanton et al., 2000a; Wegner, Schneider, Knutson, \& McMahon, 1991).

Thus, much of the research on the emotional approach strategies of processing and expression suggests that emotion-focused coping strategies are not inherently adaptive or maladaptive; the effectiveness of a given strategy for dealing with a stressful event may depend on factors such as general coping preferences, aspects of the situation, the specific emotion being expressed or processed, the timing of emotional expression or processing relative to the occurrence of the stressor (e.g., continuing to deal with emotions long after an event has occurred, which may lead to distress), and the individual's level of adjustment prior to the onset of the stressor (e.g., degree of depressive symptoms) (Stanton et al., 2000a). Additionally, coping strategies ought to be considered in a broad context as they interact with the appraisal of the situation. As previously mentioned, situations perceived as controllable may be best dealt with by using problem-focused strategies, whereas during situations appraised as uncontrollable, it may be more advantageous to use emotion-focused strategies to deal with one's distress, since attempts to change an unalterable situation may only create further distress (Zakowski et al., 2001). Appraisals and coping interact to predict the amount of distress an individual experiences in response to an event. In this regard, appraisals of low control combined with high levels of emotion-focused coping, and high control appraisals combined with low levels of emotion-focused coping, were associated with a low amount of distress in response to stressors (Zakowski et al., 2001). In effect, emotion-centred 
styles of coping may only be ineffective when used to deal with stressors that are appraised as controllable.

Inconsistencies in the findings regarding the adaptive nature of emotion-focused coping (e.g., findings of emotion-focused coping being associated with distress, versus findings of the beneficial effects of emotional approach) may be due to a number of factors related to its measurement (Stanton et al., 1994). First, disparate methods of emotion-focused coping, such as denial (indicating avoidance of a stressor) and social support seeking (indicating approach toward a stressor) are frequently combined. As well, scales intended to measure emotional coping often contain items that are highly correlated with items assessing psychopathology, such as those that involve feelings of distress (e.g., becoming tense) or self-denigration (e.g., focusing on one's own inadequacies; Stanton et al., 2004; Stanton et al., 2000b). Such confounding may account for the high correlations that have been found between emotion-focused coping and psychopathology.

Alterations in appraisals and coping among abused women. Women in abusive relationships may display altered appraisals of events, pertaining to both the relationship, and factors outside of the relationship. It is generally accepted that there is a continuum of awareness among women in abusive relationships, such that some women are not aware that they are in an abusive relationship, and perceive their partner's behaviours as relatively benign, or as normal reactions to conflict (Pipes \& LeBov-Keeler, 1997). Age and experience in relationships may be important factors influencing whether behaviours are perceived as abusive. For example, both adolescent girls and boys had difficulty delineating boundaries between normal playing, harassment, and abuse. Moreover, in the 
same study females suggested that sometimes women may want men to hit them, and may perceive such acts as a reflection of love or commitment (Johnson, Frattaroli, Campbell, Wright, Pearson-Fields et al., 2005). Consistent with this, undergraduate students in physically abusive relationships appraised restrictive and dominating behaviours as less controlling compared to those not in abusive relationships (Ehrensaft \& Vivian, 1999). As these women do not perceive certain behaviours as abusive, they may be less likely to end the relationship, or to take steps toward changing the negative behaviours. The apparent inability for these women to define their situation as abusive may reflect a strategy for blunting the emotional distress associated with such an experience.

Given the aforementioned factors, in studying intimate partner abuse it is important to consider the association between the reported experiences of researcherdefined abuse using standard scales, and actual perceptions of abuse by women in such relationships. To this end, Wagner and Mongan (1998) asked abused and non-abused women to define whether they considered each of the behaviours on the Conflict Tactics Scale (Straus, 1979), a commonly used instrument for assessing relationship violence, to be abusive. It was found that although there were no differences in ratings of the majority of physical abuse items, women who self-reported abuse (regardless of the type of maltreatment experienced) considered a greater number of emotionally abusive behaviours as offensive compared to non-abused women. In particular, behaviours such as insulting/swearing, sulking, saying something to spite the other person, and threatening to hit or throw something, tended to be endorsed as abusive more often by women who self-reported violence than those who did not. However, experience of abuse was 
indicated by only a single item inquiring as to whether women had ever been physically, sexually, or emotionally abused in their lifetime. Thus, this study was unable to differentiate between abuse that occurred in a relationship, and abuse that may have happened earlier in life (e.g., in childhood), did not distinguish currently abused women from women who had ended an abusive relationship, and did not differentiate between types of maltreatment experienced. Finally, women responded to each statement in a dichotomous (yes/no) format, and thus were not able to indicate the degree to which they perceived each behaviour as abusive, only whether it was abusive or not.

As previously mentioned, coping with stress is a continual process of appraisals and reappraisals (Zakowski et al., 2001). In dealing with relationship conflicts, individuals may reinterpret or reappraise events, changing the meaning of a situation to render it more benign and therefore less stressful. For example, women in abusive relationships may choose to focus on the love and affection received in the relationship rather than the abuse, or they may focus on individuals or situations that are worse than their own (Herbert et al., 1991). One study found that abused women utilized coping strategies that enabled them to view their relationship in a more positive light, for example, reporting that their relationship was not as bad as it could be (Herbert et al., 1991). As well, college women in highly committed relationships who experienced high levels of physical violence during conflicts in their relationship frequently reported exceptionally violent behaviours as instances of "joking around" (Arriaga, 2002). Women in committed relationships may be being less likely to perceive violent acts as abusive, and may reinterpret such acts as being non-malevolent. After all, these women are likely to be motivated to resolve the cognitive discord that occurs when notions of commitment 
to a relationship are at odds with ideas of violence (Rosen, 1996). Thus, positive reframing of abusive events may be detrimental, since it allows the seriousness of the abuse to go unchallenged.

However, positive reappraisals may only occur up to a point, such that once abuse reaches a certain level of severity, it is less likely that women will be able to justify altering their perceptions of malevolent behaviours. Along this line, it was found that the more severe verbal abuse women experienced, the less they were capable of appraising their relationships in a positive light. This finding indicates a potentially more severe psychological impact of verbal or emotional abuse compared to physical aggression, for which there was no change in appraisals with increased frequency or severity (Herbert et al., 1991; Waldrop \& Resick, 2004). An increase over time in the frequency of abuse was associated with entering a shelter, indicating that increasing abusive episodes may necessitate the utilization of more active forms of coping (Waldrop \& Resick, 2004). Further, a longitudinal study found that it was the perception of an increase in the frequency or severity of violence rather than an increase per se that predicted women's intentions to end an abusive relationship (Pape \& Arias, 2000). As well, the effect of perceived increases in violence on intent to leave an abusive relationship was mediated by attributions of the abusive partner as responsible and blameworthy, such that women were more likely to leave increasingly violent situations if they appraised their partner as responsible for the violent behaviour. Thus, as appraisals of the situation change, active coping strategies (e.g., attempts to leave the abusive relationship) may be employed, although this may depend upon a number of other factors. 
In addition to altered appraisals of events, women experiencing abuse may utilize ineffective strategies to deal with stressors, which can have deleterious effects on wellbeing. Importantly, lack of reliance on active, problem-focused coping strategies has been identified as a key factor in the relationship between abuse and mental health. Straight and colleagues (2003) found that college women in psychologically abusive relationships who used low levels of active or approach coping (e.g. problem solving) to deal with stressors showed increased binge drinking and negative health perceptions as the degree of abuse experienced increased. It was suggested that those women who utilized active coping strategies may have been more effective at diffusing distress, and thus less likely to engage in health risk behaviours. Although ineffective coping strategies are associated with the occurrence of abuse, it is unclear from these reports whether the experiences of abuse led to altered coping, or whether such coping styles influenced the choice of dating partners (Straight et al., 2003).

It is important to note that abused women may not have an overall ineffective style of coping. The contextual variability surrounding coping ought to be considered. In line with previous research on the effects of the controllability of an event on the type of coping used (e.g., Zakowski et al., 2001), it was suggested that although women may have a preferred strategy of coping, they may alter that strategy depending upon the context. In this respect, problem-focused strategies may be used in certain settings (e.g., the workplace) whereas emotion-focused or avoidant strategies may be used in the context of relationship conflicts (Waldrop \& Resick, 2004). As well, coping strategies used may depend upon a number of other factors outside of the relationship. For example, women who had experienced violence in their family of origin, and those who held more 
traditional attitudes about gender roles, were less likely to use active coping strategies to deal with violence within their relationships (Mitchell \& Hodson, 1986). Indeed, childhood stressors are particularly influential in determining the types of coping strategies used in adulthood. For example, sexually assaulted undergraduate women with a history of childhood sexual assault were more likely to use disengagement coping strategies to deal with the assault, compared to abused women without such a history (Gibson \& Leitenberg, 2001).

It was found that abused women relied on emotion-focused and avoidant strategies of coping, whereas they were less likely to use active strategies (e.g., reframing of stressful events) (Bernhard, 2000; Clements \& Sawhney, 2000; Kemp et al., 1995; Valentiner, Foa, Riggs, \& Gershuny, 1996). As well, they were unlikely to see alternatives to remaining in the abusive relationship (Clements \& Sawhney, 2000). One such passive coping strategy that is frequently used by women in abusive relationships is self-blame. In discussing this coping strategy, some have distinguished between blame as a modifiable aspect of one's behaviour (e.g., should not have talked back) and blame of one's character (e.g., personal incompetence) (Andrews \& Brewin, 1990). In particular, characterological blame frequently occurred among women in abusive relationships who also had experienced physical or sexual abuse in childhood, whereas women without such past experiences were more likely to blame some aspect of their behaviour (Andrews \& Brewin, 1990). A tendency to engage in self-blame was particularly prevalent among abused women who believed, unrealistically, that they were in control of their partner's abusive behaviour, and thus felt personally responsible when their attempts at control were unsuccessful (Rosen, 1996). In this regard, illusions of control, 
and feelings of hopefulness (i.e., holding out hope that if they persevered, the relationship would improve) were commonly reported by women in abusive relationships. Attributions of blame, however, may change over time and/or with situational factors; although women tended to blame themselves for violence experienced within the relationship, once the relationship ended, blame tended to shift from the self to the former partner (Andrews \& Brewin, 1990). As well, attributions of blame were more likely to shift from the self to the partner when violence increased in severity (Miller \& Porter, 1983). Self-blame is particularly damaging, leading to low self-esteem, depression, and hopelessness (Clements, Sabourin, \& Spiby, 2004; Herbert, Silver, \& Ellard, 1991).

Although passive strategies are frequently used by abused women, they are generally ineffective in dealing with violence. For example, it was found that placating and resisting violence, strategies that were used most frequently early in abusive relationships to avoid violence, were the least effective in ameliorating the abusive behaviour (Goodman, Dutton, Weinfurt, \& Cook, 2003). Some studies have suggested that abuse reduces the victim's ability to cope effectively. Evidence of the direct effects of abuse on coping is supported by the finding that once women left an abusive relationship, the use of emotion-focused coping strategies decreased over time, whereas the use of problem-focused strategies increased (Lerner \& Kennedy, 2000).

Certain coping strategies may influence the relationship between abuse and wellbeing. For example, although abused women were more likely to use passive rather than active strategies to cope with violence, those who did report the frequent use of problemfocused coping strategies to deal with abuse were less likely to report dysphoric symptoms than those who used emotion-focused strategies, such as self-blame and 
avoidance (Clements \& Sawhney, 2000). Coping strategies and dysphoria may operate in a reciprocal fashion whereby dysphoric symptoms increase as a result of ineffective coping, and greater dysphoria leads to the persistent use of ineffective coping strategies (Clements \& Sawhney, 2000). It may well be the case that depression interferes with the use of effective problem-solving abilities among abused women, thus impeding attempts to leave such relationships (Arias, Lyons, \& Street, 1997). As well as depression, greater use of emotion-focused coping (both in absolute terms as well as in reference to problemfocused coping) was associated with greater levels of PTSD symptomatology among abused women (Arias \& Pape, 1999).

Although much of the research has shown that problem-focused coping strategies, when used in the context of abuse, are more adaptive, this may not always be the case. Indeed, problem-focused coping may be ineffective, and even harmful, in certain situations. In line with the theory of goodness-of-fit between appraisals and coping strategies (e.g., Zakowski et al., 2001), in situations of abuse that are perceived as uncontrollable, attempts to address the source of the problem may be in vain, potentially leading to feelings of hopelessness and despair. As discussed earlier, when one does not see a means of altering a problem, the use of problem-focused coping may actually serve to increase distress. Particularly in the case of physical abuse, women may respond to the maltreatment with depression when they feel that they cannot control the event (Arias et al., 1997). Other factors, including social support, may determine the degree to which problem-focused coping confers benefit to the mental health of abused women (Kocot \& Goodman, 2003). In addition, active strategies may vary in effectiveness when used in an abusive relationship, depending upon the context in which they are used. For example, 
taking action to leave an abusive relationship (e.g., leaving the partner or entering a shelter) may differ from using active strategies while still in the relationship (e.g., arguing with the partner, calling the police) (Waldrop \& Resick, 2004). Thus, the specific contextual factors in which coping strategies are used must be taken into consideration when determining their efficacy.

In line with previous reports of the potential positive effects of emotion-focused coping (e.g., Stanton et al., 1994; Stanton et al., 2000a), avoidant or emotion-focused coping strategies used by women in abusive dating relationships (e.g., minimizing the seriousness of violent encounters, forgetting, or denying that the incident was abusive) may sometimes serve to attenuate the harmful emotional effects of abuse, at least in the short term (Rosen, 1996). Accordingly, it was found that distancing (reducing the magnitude of a problem or making light of it as opposed to actively avoiding the conflict) was associated with a lower frequency of physical aggression (Carey \& Mongeau, 1996). That is, women who tend to put conflicts in perspective, rather than avoiding them, may be better able to avoid escalation of violence. Thus, it appears that initially, avoiding or making light of conflicts in a relationship may be beneficial in avoiding abusive encounters, although this response may become maladaptive over time. Indeed, although such coping methods may be advantageous in the short term (i.e., by reducing the emotional stress associated with the abuse), avoidant strategies allow the potential danger of abuse, as well as women's fantasies about the non-serious nature of the abusive situation, to go unchallenged and unacknowledged, thus leading to the possibility of further physical and emotional harm (Rosen, 1996). Whatever the case, it seems that disengaged or avoidant coping was associated with PTSD symptoms (in particular, the 
avoidance cluster of symptoms) among abused women (Kemp et al., 1995), supporting the contention that these coping methods were ultimately ineffectual.

\section{Social Support}

Social support is generally divided into structural and functional measures (Helgeson, 2003). Structural measures involve the existence and quantity of social relationships and roles, and reflect the amount of contact or number of people individuals generally have contact with. Functional support, in contrast, consists of the amount of perceived emotional, informational and instrumental support received by others, and is generally the type of support reported on questionnaire measures. Social support can serve multiple functions across a variety of situations and circumstances. It can be beneficial to health outcomes, as evidenced by, for example, positive effects on immune system functioning (Miyazaki, Ishikawa, Iimori, Miki, Wenner et al., 2003) and decreased health care utilization (Kouzis \& Eaton, 1998). Social support can also act in a protective capacity against mental health symptoms. For example, among married couples, depression was inversely associated with both relationship quality and amount of positive social contact with individuals other than the spouse (Beach, Arias, \& O'Leary, 1986). Support can diminish feelings of hopelessness in individuals experiencing significant distress, such as that of a terminal illness (e.g., Akechi, Okamura, Yamawaki, \& Uchitomi, 1998; Johnson, Alloy, Panzarella, Metalsky, Rabkin et al., 2001), and can facilitate the use of effective strategies for coping with adverse life events, thereby decreasing susceptibility to mental illness (Arias et al., 1997; Thoits, 1986). Indeed, problem-focused coping was associated with fewer symptoms of depression and PTSD 
among battered women who experienced high levels of perceived social support, compared to those with less support (Kocot \& Goodman, 2003).

The findings regarding social support among abused women have been inconsistent. In one study, abused women reported lower levels of partner support than non-abused women, as would be expected given their experiences of maltreatment; however, there were no differences between abused and non-abused women in reported levels of social support from other sources (e.g., friends) (Carlson, McNutt, Choi, \& Rose, 2002). As well, the presence or absence of social support reported by university women did not predict the occurrence of abuse in their dating relationships (Carey \& Mongeau, 1996). In contrast to these reports, others found that compared to non-abused women, abused women perceived lower levels of social support from friends and family, even though they did not differ greatly in the actual amount of contact with friends and family (Smith, Thornton, DeVellis, Earp, \& Coker, 2002). Thus, although abused women may have contact with individuals other than their partner on a frequent basis, they may feel that they cannot rely on others for support and advice.

Greater severity of abuse was associated with lower levels of support from friends, but interestingly, this was most prominent when abused women attempted to talk about the abuse to others (Mitchell \& Hodson, 1983). Supporting this, it was reported that although abused women might seek social support, they often experienced negative reactions to their situation from their social network and as such, perceived such sources of support to be inadequate (Andrews \& Brewin, 1990). It was suggested that women in severely abusive relationships may either be intentionally isolated from social contacts by their partner, or that friends and family members may become less willing or able to 
provide support once violence escalates (Dobash \& Dobash, 1998; Waldrop \& Resick, 2004). As well, it has been proposed that when faced with severe stress, disillusionment accompanying the negative experience may alter one's perceptions of others' helpfulness, leading to a decline in the perceived availability of support, which may then adversely affect well-being (Kaniasty \& Norris, 1993). It is equally possible that the reduced feelings of social support reported may not result from the failure of others to acknowledge and help their situation, but from women's reluctance to disclose information about the abuse to family and friends. Failure to divulge information about an abusive situation may occur for a number of reasons, including feelings of shame and embarrassment about remaining in such a situation, the desire to avoid having to explain themselves, or the desire for family and friends to retain a positive image of their partner (Rosen, 1996). Indeed, in a sample of university women in dating relationships, over one third of women omitted information about their experiences of abuse when disclosing such aspects of their intimate relationships with friends and relatives (Dunham \& Senn, 2000). As well, friends and family may cease being supportive if they feel that the woman is not making attempts to terminate an abusive relationship (Waldrop \& Resick, 2004).

If perceived as positive, social support may provide a buffer against mental health symptoms among abused women. In this regard, it was reported that social support played a mediating role in the relationship between abuse (physical, sexual, or psychological) and both physical and mental (depression, PTSD, anxiety) health, such that higher levels of emotional support were associated with greater mental well-being and self-perceived health status among abused women (Coker et al., 2002, 2003). Among 
battered women living in the community, it was the total number of supporters, in particular those who had not experienced abuse themselves and who could thus provide hope for a future without relationship violence, that accounted for greater self-esteem and lower depression (Levendosky, Bogat, Theran, Trotter, von Eye et al., 2004). It was suggested that abuse may be associated with estrangement from others, as well as a reduced sense of self-esteem or self-worth (Coker et al., 2003). The presence of social or emotional support may serve to offset these negative effects by enhancing psychological health and improving coping strategies, such that women are able to consider viable alternatives to remaining in the abusive relationship.

In contrast to the findings concerning the beneficial effects of social support on the well-being of women in abusive relationships, it was reported that active social support seeking among abused women in shelters was unrelated to dysphoric symptoms (Clements \& Sawhney, 2000). As well, a high level of perceived social support from one's spouse was, in one report, associated with increased depressive symptomatology among abused women, relative to abused women who reported less support (Arias et al., 1997). Thus, women who perceive their partner as a source of positive social support may respond to victimization with increased symptoms of depression relative to those who do not have such positive perceptions of their partner (Arias et al., 1997).

It has been suggested that social support may alter one's appraisal of a stressor such that it is perceived as less threatening. Social support may function as "coping assistance," aiding a person to change the situation or the emotional reactions to it (e.g., by creating diversions from a stressor, providing advice, or aiding reinterpretation to make a situation seem less threatening; Thoits, 1986). In this regard, Lazarus and 
Folkman (1984) indicated that social support is an important resource that precedes and influences coping. However, this relationship may depend upon appraisals of the situation. For example, when stressors were perceived as controllable, social support facilitated adaptive coping, but when stressors were appraised as uncontrollable, support was not associated with the coping strategy used (Valentiner, Holahan, \& Moos, 1994). In the case of abuse, support may reinforce an abused woman's perceptions that she will be successful in coping with the abusive relationship, thus reducing feelings of hopelessness and dejection (Kocot \& Goodman, 2003). For example, women who had used cognitive restructuring to minimize or rationalize their partner's behaviour were more likely to reappraise the situation as non-benign after hearing the supportive opinions of others (Waldrop \& Resick, 2004). The presence of support may also alleviate the appraisal of stressors as threats (Kocot \& Goodman, 2003) and may reduce feelings of self-blamè among abused women (Arias et al., 1997).

In addition to altered appraisals, social support may be related to the coping strategies employed, and may partially determine the extent to which coping strategies used by abused women confer benefit to mental health. In particular, it has been suggested that women who feel that they are supported by others may be more likely to utilize active, problem-focused coping efforts to deal with the abuse (Kocot \& Goodman, 2003). Thus, social support may facilitate active coping strategies. Conversely, under conditions of low perceived social support, or social isolation, problem-focused coping efforts may be ineffective, or even detrimental to mental health (Kocot \& Goodman, 2003). As well, concrete support, for example, in the form of money or a shelter, may help abused women directly alter their circumstances (Arias et al., 1997), thus aiding in 
the use of adaptive, problem-focused styles of coping. Women who receive negative feedback while attempting to obtain support from friends and family may be more likely to give up using active strategies in favour of avoidant strategies (Mitchell \& Hodson, 1983).

In addition to the level of perceived support, the nature of the support women receive is critical to well-being. In this regard, when support is perceived as unambiguous and consistent with women's actions, it may facilitate active coping efforts and reduce distress associated with taking action. In contrast, advice perceived as ambiguous and inconsistent with a woman's actions may exacerbate the emotional distress associated with active coping efforts. Greater use of problem-focused coping among abused women was associated with fewer symptoms of depression and PTSD, but only when women had higher levels of perceived social support, and when such support was unambiguous and consistent with their active coping efforts (i.e., attempting to leave the abuser; Kocot $\&$ Goodman, 2003). Together, it seems that social support mediated or moderated the effects of other coping strategies with respect to the impact of abuse on mental health. Neuroendocrine Variations associated with Abuse Like other stressors, abuse may result in alterations of HPA activity. Under normal conditions, stressor exposure may instigate the release of corticotropin-releasing hormone (CRH) from the hypothalamus. It appears that $\mathrm{CRH}$ released from neuronal terminals situated in the median eminence of the hypothalamus stimulates the release of adrenocorticotropic hormone (ACTH) from the pituitary into the blood stream. The circulating ACTH, in turn, stimulates the synthesis and release of the glucocorticoid hormone, cortisol, from the adrenal cortex (Sapolsky, Romero, \& Munck, 2000; Welberg 
\& Seckl, 2001). Feedback inhibition occurs through the binding of circulating glucocorticoids to receptors in the hypothalamus and hippocampus, where they inhibit the release of CRH, thereby attenuating the HPA response (Brown, Rush, \& McEwen, 1999; Keller-Wood \& Dallman, 1984). Feedback is related to the amount of cortisol present in the brain, particularly the hippocampus, such that high or increasing concentrations of cortisol increase inhibition of CRH-secreting cells in the hypothalamus, whereas lower or decreasing concentrations allow for increased CRH release (Stokes, 1995). Although cortisol is protective under stressful conditions (e.g., by increasing the body's energy supplies and suppressing the immune system in order to prevent damage by overactivation of the primary defence systems), upon repeated exposure and/or severe distress this system may become overly taxed, leading to a breakdown in the normal feedback inhibitory control, and increasing vulnerability to pathology, a phenomenon known as "allostatic overload" (McEwen, 2000).

In addition to being a prime stimulant of the HPA response, $\mathrm{CRH}$ in other brain regions is believed to contribute to anxiety and affective disorders (Arborelius, Owens, Plotsky, \& Nemeroff, 1999). In this regard, CRH and its receptors have been localized in brain areas known to be involved in the cognitive, affective, and autonomic responses to stressors, including the prefrontal cortex and amygdala, as well as in brainstem nuclei (De Souza, 1995; Sanchez, Young, Plotsky \& Insel, 1999; Sauvage \& Steckler, 2001). When administered centrally to laboratory animals, $\mathrm{CRH}$ produces behavioural and physiological changes that are analogous to those that occur during exposure to a stressor, and which are reminiscent of symptoms of depression and anxiety in humans (e.g., increased heart rate, suppression of exploratory behaviour, and disruption of feeding and 
reproductive behaviours) (e.g., Korte, Bouws, \& Bohus, 1993; Momose, Inui, Asakawa, Ueno, Nakajimi et al., 1999). As well, increased CRH-like immunoreactivity was found in the cerebrospinal fluid (CSF) of drug-free depressed patients compared to patients with other psychiatric disorders and healthy controls (Nemeroff, Widerlov, Bissette, Walleus, Karlsson et al., 1984), as well as in the postmortem brain tissue (frontal cortex) of depressed patients and suicide victims, who most likely suffered from clinical depression (Arato, Banki, Bissette, \& Nemeroff, 1989). Additionally, in a recent study, it was reported that $\mathrm{CRH}$ was elevated in the frontopolar and dorsomedial prefrontal cortex of depressed suicides (Merali, Du, Hrdina, Palkovits, Faludi et al., 2004). The increase was accompanied by down-regulation of $\mathrm{CRH}_{1}$ receptors (thought to be associated with stressor reactivity), but not $\mathrm{CRH}_{2}$ receptors (whose function with regard to stress is less well defined). Consistent with the CSF and postmortem findings, treatment regimens that reduce depressive symptoms, including many antidepressant medications as well as electroconvulsive therapy, have been reported to reduce CRH concentrations in the CSF (Nemeroff, Bissette, Akil, \& Fink, 1991; Veith, Lewis, Langohr, Murburg, Ashleigh et al., 1993; De Bellis, Gold, Geracioti, Listwak, \& Kling, 1993). In fact, on the basis of such findings there have been attempts to develop antidepressant medications based on selective antagonism of $\mathrm{CRH}$ receptors (Zobel, Nickel, Kunzel, Ackl, Sonntag et al., 2000). Reduced levels of plasma and salivary cortisol have repeatedly been shown among individuals with PTSD, suggesting dysfunction of feedback processes responsible for glucocorticoid regulation (Yehuda, 2002) or CRH hypersecretion and resultant downregulation of CRH receptors (Heim, Owens, Plotsky, \& Nemeroff, 1997). Indeed, combat veterans with PTSD were found to have higher cerebrospinal fluid (CSF) levels of CRH 
than healthy controls (Baker, West, Nicholson, Ekhator, Kasckow et al., 1999; Bremner, Licinio, Darnell, Krystal, Owens et al., 1997).

Although CRH hypersecretion appears to be a common feature of both major depression and PTSD, other neuroendocrine aspects of depression and PTSD are vastly different. In particular, depression is associated with hypersecretion of cortisol, and diminished suppression (or early escape from suppression) of cortisol in the dexamethasone suppression test, whereas PTSD is associated with hyposecretion of cortisol as well as enhanced cortisol suppression on the dexamethasone test (Heim et al., 1997). Lower cortisol among individuals with PTSD is consistent with reports of enhanced negative feedback of cortisol, indicated by an increase in the quantity and sensitivity of glucocorticoid receptors in PTSD patients (Yehuda, Lowy, Southwick, Shaffer, \& Giller, 1991; Yehuda, Boisoneau, Mason, \& Giller, 1993; Yehuda, Giller, Levengood, Southwick, \& Siever, 1995). Consistent with the findings in male veterans with combat-related PTSD, women with a history of childhood and/or adolescent sexual abuse had enhanced suppression of cortisol in the dexamethasone suppression test (Stein, Yehuda, Koverola, \& Hanna, 1997). Likewise, victims of domestic violence with a diagnosis of PTSD exhibited a hypersuppression of cortisol in response to dexamethasone, indicating enhanced negative feedback inhibition, while those with comorbid PTSD and depression displayed significantly less cortisol suppression (Griffin, Resick, \& Yehuda, 2005). Furthermore, cortisol and ACTH levels in response to a psychosocial laboratory stressor were positively related to the severity of depressive, but not PTSD, symptoms (Heim, Newport, Wagner, Wilcox, Miller, et al., 2002). 
Several reports concerning the effects of trauma on cortisol levels have been inconsistent with the aforementioned findings. For example, women with PTSD as a result of childhood sexual abuse exhibited heightened urinary cortisol levels (Lemieux \& Coe, 1995), suggesting the possibility of different biological phenotypes of PTSD. Alternatively, other factors such as time since the traumatic event may be important in the cortisol-PTSD relationship, or differences may result from inconsistencies in measurement of the hormone (Yehuda, 2002). Furthermore, some of the discrepancies in findings may result from differences in illness severity in the populations studied: lower cortisol levels were found among severely ill, hospitalized patients, while higher levels were found in outpatients (Rasmusson, Vythilingam, \& Morgan, 2003). An assessment of the 24-hour circadian rhythm of cortisol of Vietnam combat veterans revealed that those with PTSD generally exhibited lower basal cortisol release, particularly in the late evening and early morning, compared to veterans with major depression and nonpsychiatric controls (Yehuda et al., 1996). On the basis of the finding of a greater range of cortisol levels throughout the day in individuals with PTSD (e.g., showing greater declines at certain points throughout the day), it was suggested (Yehuda et al., 1996) that HPA axis activity in PTSD may be over-responsive to stressor-related signals, whereas depression may be characterized by minimal responsiveness to such cues. The lower background activity in PTSD may allow greater potential for maximal responsiveness to the environment (Yehuda, 2002). Others have found that cortisol hypersecretion in depression is only evident at certain times during the day, particularly in the morning, indicating that cortisol regulation in depression may be best characterized as dysregulated 
rather than consistently high release (Casper, Swann, Stokes, Chang, Katz et al., 1987; Yehuda et al., 1996).

Experiences of intimate partner abuse may result in long-term alterations of the HPA functioning. However, the few studies that have assessed this possibility have yielded conflicting results. Inconsistencies between studies may be a result of variations such as the time since the abused women had been out of the relationship, and the location of sampling (normal environment versus laboratory), factors that may have a profound impact on neuroendocrine activity.

Research on early childhood trauma has indicated that experiences of distress, including abuse, may result in long-term alterations of neuroendocrine functioning, increasing the risk of developing stressor-related disorders such as depression (e.g., Heim \& Nemeroff, 2001, 2002; Penza, Heim, \& Nemeroff, 2003). Adult trauma appears to have similar effects. For example, one study reported that women who had previously experienced physical or sexual abuse within an intimate relationship demonstrated low morning basal levels of plasma cortisol compared to non-abused women, although basal cortisol was unrelated to the degree of PTSD or depressive symptoms reported (Seedat, Stein, Kennedy, \& Hauger, 2003). Griffin et al. (2005) reported low morning baseline levels of cortisol in a sample of battered women with PTSD or comorbid PTSD and depression, compared to non-abused controls and abused women without a diagnosis of psychopathology. Conversely, other investigators reported that abused women, particularly those with experiences of physical assault, exhibited heightened evening (but not morning) levels of cortisol, which was unrelated to level of depression or PTSD (Pico-Alfonso, Garcia-Linares, Celda-Navarro, Herbert, \& Martinez, 2004). Furthermore, 
a recent study of women who had experienced intimate partner violence suggested that those with PTSD had higher cortisol levels throughout the day compared to abused women without PTSD, even after controlling for age, depression, and severity and duration of abuse. In fact, even among women with remitted PTSD, cortisol levels remained high, indicating that PTSD may result in long-term alterations in HPA activity (Inslicht, Marmar, Meylan, Metzler, Hart et al., 2006).

In addition to altered basal neuroendocrine activity, abused women may show a dysfunctional HPA response to stressors. For example, women in abusive dating relationships, particularly those with high levels of PTSD symptoms, exhibited a heightened cortisol response to a video regarding abuse, if they were asked beforehand (i.e., 'primed') to recall their history of traumatic events (Matheson, 2003). Conversely, women exhibited lower cortisol levels following an experience of rape if they had a history of prior sexual or physical assault (Yehuda, Resnick, Schmeidler, Yang, \& Pitman, 1998).

\section{Present Study}

Coping with stressful life experiences is known to be an important factor in determining whether psychopathology would be evident (Anisman \& Matheson, 2005). Likewise, it is possible that the incidence of depressive and PTSD symptoms among abused women is related to a number of other factors associated with appraisal and coping processes. There is, indeed, evidence consistent with a role for appraisals, coping, and social support in the abuse-pathology relationship among battered women, as well as for a relationship between abuse and physiological alterations (i.e., variations in HPA activity), but much less information is available regarding women in dating relationships. 
Yet, it is important to be able to assess accurately the processes related to abuse and the abuse-pathology relationship in order to develop appropriate intervention strategies that can be instituted at an early stage before abuse escalates, and before pathology has taken root.

In light of such considerations, the present investigation examined abuse in dating relationships of female students to ascertain the nature of the abuse encountered, and the ramifications of this abuse in terms of women's functional and psychological well-being. Although it is recognized that males may also be victims of intimate partner violence, such aggression may occur for different reasons (e.g., seeking partner's attention, selfdefense; Fiebert \& Gonzalez, 1997; Saunders, 1986), and the affective consequences (e.g., depression, anxiety, suicidal behaviour) are generally of lower severity (O'Leary, 1999). As well, men and women tend to report different types of violence: although overall, the frequency of aggression was the same in both genders, women were more likely to report experiences of sexual aggression, whereas men were more often victims of psychological maltreatment (Harned, 2001). Thus, the present investigation focused on male-to-female aggression.

The effects of abuse on the development of psychopathology (e.g., symptoms of depression and PTSD) may be related to interactions among appraisal processes, coping, and psychological characteristics. The aims of the present investigation were to examine (1) the psychological (symptoms of depression, PTSD) and neurobiological (salivary cortisol) correlates of abuse, (2) appraisals, coping styles, and social provisions (support) among women in psychologically, sexually, and physically abusive relationships, (3) the extent to which such variables mediate the effects of abuse on symptoms of depression 
and PTSD, and (4) whether the cortisol response to a challenge varies in response to abuse and the presence of PTSD symptoms.

\section{Study 1}

As indicated earlier, women in abusive relationships frequently report a high incidence of depression and PTSD relative to those in healthy relationships (e.g., Golding, 1999). Although various coping strategies may be employed to contend with their situation, these strategies may not be consistently effective in buffering women in abusive relationships against psychological pathologies. Indeed, several studies indicated that women in abusive relationships often utilize unproductive means of dealing with conflict (e.g., Clements \& Sawhney, 2000; Straight et al., 2003). Furthermore, women in abusive relationships may experience lower levels of social support, although the presence of support may help to reduce the adverse effects of abuse on well-being (e.g., Coker et al., 2002, 2003). Study 1 was conducted to discern the impact of abuse in dating relationships on symptoms of depression and PTSD, and to assess the potential mediating effects of coping and social support on such symptoms. It was hypothesized that compared to non-abused women, those in abusive relationships would report lower levels of social support and greater use of ineffective means of coping with stressful events within their relationship (specifically, greater use of emotion-focused and avoidant strategies, and less use of problem-focused strategies), and that they would exhibit greater symptoms of depression and PTSD. In addition, it was hypothesized that coping and social support would mediate the relationship between abuse and symptoms of psychological distress. Specifically, women in abusive relationships who experience lower social support, and who tend to utilize ineffective coping strategies, may in turn be 
more vulnerable to symptoms of depression and PTSD. The model in Figure 1 was used to assess these hypotheses.

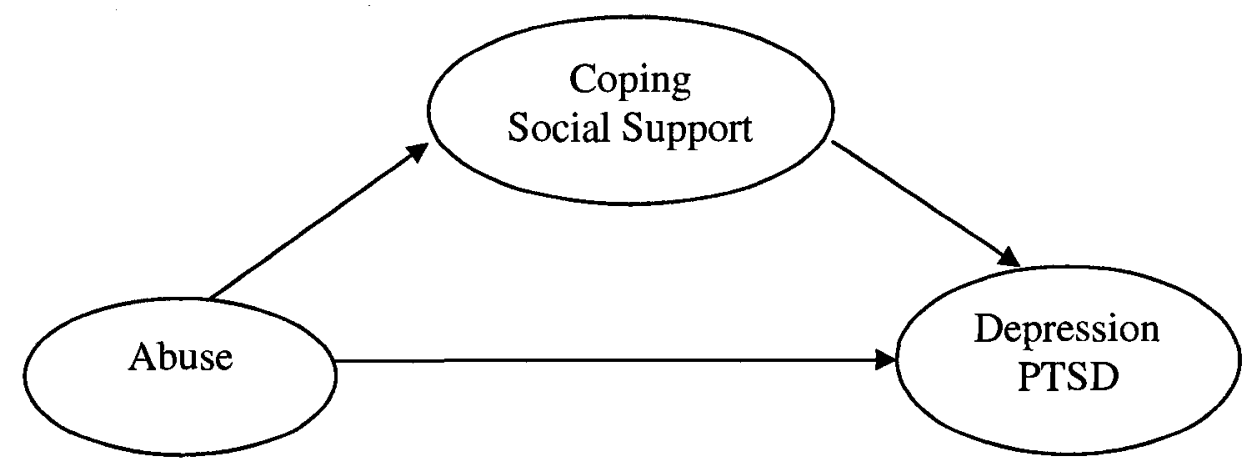

Figure 1. Mediating Effects of Coping and Social Support on the Relationship between Abuse and Symptoms of Depression and PTSD

Furthermore, it was of interest to determine whether circulating stress hormone levels (cortisol measured in saliva) differed as a function of being in an abusive relationship. It will be recalled that although stressful events are associated with elevated levels of cortisol, among individuals who encountered traumatic events and exhibited PTSD symptoms, levels of this hormone may be diminished (e.g., Yehuda, Golier, \& Kaufman, 2005; Yehuda et al., 1996). Indeed, when challenged with CRH, levels of ACTH were reduced among women that suffered early life trauma and then subsequently fell into depression. Yet, when challenged psychologically, these women exhibited an increase of HPA activity, suggesting potential activation of processes that affected HPA functioning, such as the amygdala and prefrontal cortex (Heim, Newport, Heit, Graham, Wilcox et al., 2000; Heim, Newport, Bonsall, Miller, \& Nemeroff, 2001). In effect, it may be that although earlier trauma reduced activity of the HPA axis, other brain regions 
(e.g., limbic areas) may be sufficiently sensitized to overcome the HPA downregulation. The findings also suggest that although stress hormone levels may be altered among individuals experiencing ongoing or previous stressors, such as abuse, it may be difficult to detect subtle changes in the HPA system without the presence of a challenge. Thus, in addition to taking a measure of baseline cortisol, the neuroendocrine response to a mild challenge was assessed in Study 1. Women were shown several potentially stressful images, and were then were asked to respond to questions based on these scenes. Samples of saliva were taken before and after the task to determine whether abused and nonabused women could be differentiated with respect to basal and stress-induced cortisol changes, and also whether any differences based on experience of abuse were dependant upon the level of PTSD symptomatology. It was proposed that abuse would be associated with a heightened cortisol response to the stressor, but that this would be most pronounced among women with high levels of PTSD symptoms.

\section{Methods}

\section{Participants}

Women from an introductory psychology course $(N=143 ; M$ age $=20.14, S D=$ 2.75) were recruited through mass testing and sign-up sheets (Appendix 1) to participate in a study on heterosexual dating relationships. The majority $(n=103,72.0 \%)$ were in their first year of studies. The others were in second year $(n=21,14.7 \%)$, third year $(n=$ $7,4.9 \%)$ or fourth year $(n=9,6.3 \%)$, or did not report year of study $(n=3,2.1 \%)$. Most participants reported being on some form of prescription medication: birth control $(n=$ $75,52.4 \%)$, anti-inflammatories $(n=4,2.8 \%)$, antidepressants $(n=3,2.1 \%)$, or other medications $(n=10,7.0 \%)$. As well, most participants had never been in therapy ( $n=$ 
$106,74.1 \%$ ), whereas 33 participants (23.1\%) had been in therapy but were no longer, and $4(2.8 \%)$ were in therapy at the time of testing.

On average, women reported having been in relationships for 17.01 months $(S D=$ $13.49 ;$ Range $=.50$ to 48 months), and time in the relationship did not differ between abusive and non-abusive relationships. Of the women who reported ethnic status, the majority ( $n=97,67.8 \%$ ) were Euro-Caucasian. Others were Middle Eastern $(n=6$, $4.2 \%)$, Black $(n=7,4.9 \%)$, Asian $(n=10,7.0 \%)$, Native Canadian $(n=1, .7 \%)$, Hispanic $(n=4,2.8 \%)$, or of mixed racial background $(n=3,2.1 \%)$.

Although the focus of the present study concerned current intimate partner abuse, the possibility of past experiences of trauma and abuse are also reported here, as such experiences may impact current well-being. The effects of traumatic events are cumulative, such that experiences occurring earlier in life (i.e., before abuse occurs) may have a significant impact on the duration and severity of stress symptoms experienced (Brewin, Andrews, \& Valentine, 2000). To this end, women completed the Traumatic Life Events Questionnaire, a measure designed to assess one's exposure to a variety of potentially traumatic events (Kubany, Hanes, Leisen, Owens, Kaplan et al., 2000). Using a typology described by Breslau, Chilcoat, Kessler, and Davis (1999), trauma history scores in relation to four discrete categories were determined, namely, experiences of a shocking or unexpected event (natural disaster, accident, living in a war zone, witnessing a violent event, experienced a life-threatening illness, miscarriage or abortion), unexpected death, witnessing negative events (life-threatening accident or illness of a loved one, witnessing family violence when growing up), and assault (robbery, injured by a stranger, experienced death threats, childhood physical assault, sexual assault, previous 
partner abuse, experienced stalking).

As indicated by the Chi square analysis in Table 1, women in abusive relationships ${ }^{1}$ reported significantly more previous experiences of shocking or unexpected events, but they did not differ in the frequency of experiences of unexpected death, witnessing of negative events, or assault.

Table 1. Frequencies (and Percentages) of Previous Traumatic Experiences among Abused versus Non-Abused Women

\begin{tabular}{lcc}
\hline & Abused (N = 58) & Non-abused (N = 85) \\
\cline { 2 - 3 } Shocking/unexpected event* & $37(63.8 \%)$ & $39(45.9 \%)$ \\
Unexpected death & $30(51.7 \%)$ & $37(43.5 \%)$ \\
Witness negative event & $33(56.9 \%)$ & $43(50.6 \%)$ \\
Assault & $39(67.2 \%)$ & $49(57.6 \%)$ \\
\hline$* p<.05$ & & \\
\end{tabular}

\section{Procedure}

Participants completed an informed consent form (Appendix 2) as well as measures assessing the current status of their relationship, abuse, social support, coping, and psychological well-being in terms of symptoms of depression and PTSD (Appendix 3). Saliva samples were taken $15 \mathrm{~min}$ after entering the laboratory, as well as $15 \mathrm{~min}$ and 30 min following the challenge. At the end of the session, women were debriefed and provided with contact information in the event that they wished to seek help associated with problematic relationships or distress symptoms (Appendix 2). All procedures were

\footnotetext{
${ }^{1}$ The method used for classifying women into abusive relationships is described below in the section on the Conflict Tactics Scale used for measuring abuse.
} 
approved by the Ethics committee of the Carleton University and met the Canadian tricouncil guidelines.

\section{Measures}

Abuse. A 39-item revised version of the Conflict Tactics Scale (CTS-R; Straus, McCoy, Hamby \& Sugarman, 1996) was used to assess the tactics intimate partners used to resolve conflicts within the past month. This period of time was considered appropriate, given the focus of the present research on dating relationships. The subscales used in the analyses were psychological aggression (consisting of verbal and non-verbal psychologically aggressive behaviours, e.g., "My partner insulted or swore at me"), physical assault (e.g., "My partner punched or hit me"), sexual coercion (behaviour that is intended to persuade the partner into unwanted sexual activity, e.g., "My partner used force (like hitting, holding down, or using a weapon) to make me have sex"), and injury (e.g., "I had a sprain, bruise, or small cut because of a fight with my partner"). All conflict tactics indices were individually determined by obtaining the average of scores on each subscale and multiplying each by its respective number of items. Responses were on a 6-point scale, from 0 (never) to 5 (more than 10 times). Scale reliability analyses using the Cronbach's alpha reliability coefficient, a measure of the internal consistency of a scale ${ }^{2}$, indicated that reliability was high for the physical assault subscale $(\alpha=.80)$, whereas that of the psychological abuse subscale was moderate $(\alpha=.69)$. In the main, the physical and psychological abuse items endorsed most frequently were relatively minor, according to the classifications of the CTS items as minor or severe by Straus and 
colleagues (1996). In fact, three of the physical abuse items classified as severe were not endorsed by any of the participants.

Reliability for the injury subscale was very low $(\alpha=.08)$. Three of the items were not endorsed by any of the participants. Again, these items ("I passed out from being hit in the head by my partner in a fight," "I went to a doctor because of a fight with my partner," and "I had a broken bone from a fight with my partner") were of a severe nature according to the above-mentioned classification. Only one participant endorsed the only remaining injury item classified as severe. Three participants indicated injury but did not endorse any of the physical assault items. Thus, it was decided to combine the injury and physical assault subscales. The reliability coefficient of the combined physical assaultinjury subscale was .80 .

Reliability for the sexual coercion subscale was low $(\alpha=.41)$. Three of the items of the sexual coercion subscale were not endorsed by any participant. These items ("My partner used force (like hitting, holding down, or using a weapon) to make me have sex," "My partner used threats to make me have oral or anal sex" and "My partner used threats to make me have sex") were of a more severe nature, according to the categorizations of Straus and colleagues (1996). The only remaining sexual abuse item considered severe ("My partner used force (like hitting, holding down, or using a weapon) to make me have oral or anal sex") was endorsed by only two participants. However, when the three items not endorsed were removed and the analysis rerun, reliability was still low $(\alpha=.47)$.

\footnotetext{
${ }^{2}$ Although a reliability coefficient of .70 is generally used for retaining a scale (Nunally, 1978), a more lenient cut-off of .60 is sometimes adopted. In particular, the number of items can adversely affect the reliability of a scale (Garson, n.d.). Thus, in cases where the number of items in a scale is low (as happens in cases where a full scale is divided into smaller subscales of only a few items each which are then assessed for reliability individually), a more lenient coefficient may be warranted.
} 
The physical and sexual abuse variables were highly skewed (sk $=4.93$ and 3.17, respectively). Psychological aggression was mildly skewed, with a value of 2.02 (SE = $.20)$, which was considered acceptable given the suggestion that skew values should be in the range of -2 to +2 (Garson, n.d.). Transforming these variables did not considerably alter the skew. The sexual and physical abuse variables were dichotomized, such that women were categorized as being in a physically or sexually abusive relationship if they indicated having experienced any physical abuse/injury or sexual abuse, respectively (i.e., a score of 1 or greater). Psychological abuse, on the other hand, was treated as a continuous variable in the analyses. The final sample of women in the present investigation comprised 85 who were not currently in an abusive dating relationship, and 58 who were. Specifically, 23 participants $(16.1 \%)$ were experiencing physical abuse/injury, and 27 (18.9\%) were in a sexually coercive relationship. In addition, 37 (25.9\%) were experiencing psychological maltreatment, defined as score of 3 or greater on the psychological aggression subscale of the CTS-R. Admittedly, a cut-off score of 3 or greater for defining psychological abuse is arbitrary; however, this was thought to be a relatively conservative criterion that favoured aggressive acts being considered in the context of a conflictual relationship (i.e., not an isolated incident) (Kuffel \& Katz, 2002). Twenty-two (15.4\%) of the abused women were experiencing more than one type of maltreatment. Indeed, the different types of abuse were highly correlated (physical and psychological, $r=.53$; sexual and psychological, $r=.46$; physical and sexual, $r=.42$, all significant at $p<.01)$. The variability of scores reflecting sexual and physical abuse and injury in women's dating relationships was relatively small. The amount of abuse reported, overall, was relatively low (physical assault/injury $M=.63 /$ month, $S D=2.12$; 
sexual coercion $M=.62 /$ month, $S D=1.61)$. Psychological aggression scores were higher and more variable $(M=2.22, S D=3.34)$.

Depressive symptoms. The 21 -item version of the Beck Depression Inventory (BDI, Beck \& Beck, 1972) was used as an indicator of depressive symptoms. Participants were asked to indicate the severity of each item for them on a scale ranging from $0-3$ (e.g., 0 = I do not feel sad; $3=$ I am so sad or unhappy that I can't stand it). Scores on the BDI ranged from 0 to 35 , with an overall mean score of $9.03(S D=6.41)$. Total scores on the BDI were summed, averaged, and multiplied by the number of items on the scale to account for missing values. Scale reliability analysis indicated high reliability for the scale $(\alpha=.83)$.

Symptoms of posttraumatic stress disorder. A 22-item version of the Impact of Events Scale-Revised (IES-R; Weiss \& Marmar, 1997) was used to assess PTSD symptoms. The IES-R assesses the PTSD symptom clusters of avoidance, intrusion, and hyperarousal. Although it has been utilized as a three-factor scale, studies using the IES-R have also used a single overall score indicating the extent to which an individual suffers from the three types of symptoms. Indeed, Weiss and Marmar (1997) reported a strong single factor solution in their initial analyses of the psychometric properties of the IES-R using a sample of emergency personnel exposed to disasters, and the reliability of this single factor structure has been replicated in Vietnam veterans (Creamer, Bell, \& Failla, 2003). Furthermore, the factors were highly correlated in the present study $(r=.65, .85$, and .59 for the correlations between intrusion and avoidance, intrusion and hyperarousal, and hyperarousal and avoidance, respectively; all significant at $p<.001)$. Thus, in the present report, item scores were summed to determine a total score for each participant. 
Participants indicated how distressing each symptom (e.g., "Any reminder brought back feelings of it") had been during the past seven days as a result of a recent or past stressful event, using a 5-point scale ranging from 0 (not at all) to 4 (extremely). This scale was highly reliable $(\alpha=.91)$. Scores on the IES ranged from 0 to 73 , with an overall mean score of $30.64(S D=16.18)$.

Coping styles. The 50-item Survey of Coping Profile Endorsement (SCOPE; Matheson \& Anisman, 2003) was used to assess strategies utilized by women to deal with conflict within an intimate relationship. This scale comprised items that assessed the use of problem-solving, cognitive restructuring, active distraction, rumination, humour, social-support seeking, emotional expression, other- and self-blame, emotional containment, passive resignation, religion, and wishful thinking. A summary of the correlations between the subscale scores is provided in Table 2 . The psychometric characteristics of this scale have previously been reported (Matheson \& Anisman, 2003). In a sample of university students (Matheson, Kelly, Cole, Tannenbaum, Dodd, et al., 2005), the coping subscales were significantly correlated with those from a commonly used scale developed by Carver, Scheier, \& Weintraub (1989), providing evidence of the scale's concurrent and construct validity. Specifically, problem-focused coping scores from the SCOPE were correlated with Carver and colleagues' active coping $(r=.56)$ and planning $(r=.59)$, cognitive restructuring was associated with positive reinterpretation and growth $(r=.51)$, social support seeking was related to social support seeking for instrumental $(r=.52)$ and emotional purposes $(r=.62)$, and emotional expression was related to venting of emotions $(r=.46)$.

Respondents endorsed whether they had demonstrated each of the behaviours as a 
way of dealing with a recent (in the past month) disagreement with their partner that they had found the most troublesome, using a 5-point scale ranging from 0 (never) to 4 (almost always). A factor analysis using an oblique rotation (specifically, direct oblimin), to allow for the possibility of correlations among factors, was performed on the subscale scores. Based on an examination of the scree plot, a 3-factor solution was obtained, which explained $60.75 \%$ of the variance. The three variables were labelled "emotionfocused coping" (comprising rumination, emotional expression, wishful thinking, and self-blame; $\alpha=.80$ ), "problem-focused coping" (containing problem solving, cognitive restructuring, social support, and active distraction; $\alpha=.67$ ), and "avoidant coping" (comprising avoidance, passivity, humour, emotional containment, and other blame; $\alpha=$ .66). Religious coping did not fall into any of the 3 factors, and was thus not included in the analyses. A score for each of the three categories of coping was obtained by averaging the scores for each set of items. There were significant correlations among the factors, as indicated in Table 3. 
N

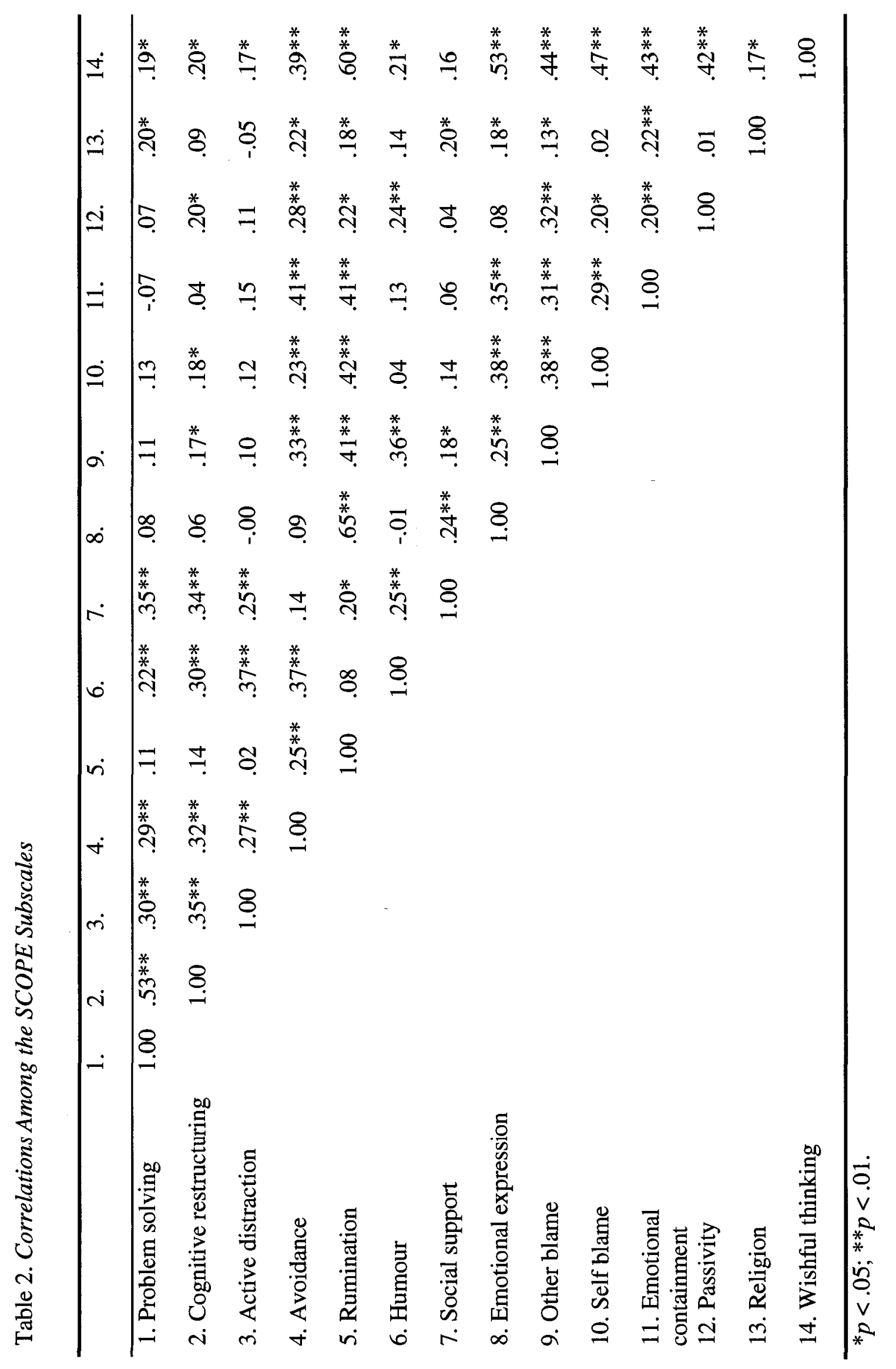


Table 3. Correlations Among the Dimensions of Coping

\begin{tabular}{llll}
\hline & 1. Problem-focused & 2. Emotion-focused & 3. Avoidant \\
\hline 1. & 1.00 & $.24^{* *}$ & $.35^{* * *}$ \\
2. & & 1.00 & $.47^{* * *}$ \\
3. & & 1.00 \\
\hline & & &
\end{tabular}

Social support. Social support was assessed using the Social Provisions scale (Cutrona \& Russell, 1987), which measures perceived social support from friends, parents, and partner (12 items each). Participants indicated extent of agreement with each item (e.g., "Are there friends you can depend on to help you, if you really need it?") using a 3-point scale, where 1 = "No", 2 = "Not sure" and 3 = "Yes." Extent of social support from each source was calculated by obtaining the mean of each of the subsets of 12 items. Scale reliability analysis indicated that the subscales were highly reliable (friends, $\alpha=.81$; parents, $\alpha=.87$; partner, $\alpha=.72$ ). The relations between support from parents and from friends $(r=.21)$, as well as that between support from partner and from friends $(r=.41)$, were significant ( $p<.05$ and $p<.01$, respectively), whereas the correlation between support from parents and from partner was not significant $(r=.11)$.

Stressor challenge. Alterations of salivary cortisol levels as a result of being in an abusive relationship were assessed at baseline and after a challenge. The first saliva sample was taken approximately $15 \mathrm{~min}$ after arrival at the laboratory to allow for acclimatization to the unfamiliar environment, while the others were taken $15 \mathrm{~min}$ and 30 min following the completion of the task. After the 15 min relaxation period during 
which participants sat comfortably, they were shown three pictures (selected from the International Affective Pictures System; numbers 6360, 9041, and 9921) depicting various stressful situations (relationship abuse, child abuse, and a fire scene). Following the viewing of each picture, participants were asked how positively and how negatively they perceived each scene to be, on a 7-point Likert-type scale. As well, they were given an abbreviated (27 item) coping scale (based on the full SCOPE) in which they were asked which strategies they would use to deal with the event in the picture. These were not analyzed for the present study, but were used instead as part of the stressor challenge. The picture task was followed by a 15 min rest period before providing the second saliva sample, and another 15 min rest period, after which participants provided the third sample. During the rest periods, participants were simply asked to relax and read a magazine if they chose to.

Cortisol determination. In healthy individuals, circulating cortisol levels are high at awakening, and decrease steadily throughout the day (Pruessner, Wolf, Hellhammer, Buske-Kirschbaum, von Auer, Jobst, et al., 1997). Thus, variations of cortisol following a challenge may be difficult to discern earlier in the day, when levels are declining. To minimize the effects of diurnal variations of hormone release, all participants were tested in the afternoon (between 2:00 PM and 5:00 PM).

Saliva samples for determination of circulating cortisol levels were collected by the participant by placing a piece of dental cotton in her mouth for approximately 2 to $3 \mathrm{~min}$, or until the cotton was thoroughly wet, after which it was inserted into a test tube. A saliva sample was collected at the beginning of every session (following a $15 \mathrm{~min}$ period of relaxation after entering the laboratory), and again $15 \mathrm{~min}$ and $30 \mathrm{~min}$ after completion 
of the task. Samples were maintained at $-80^{\circ} \mathrm{C}$ until subsequent cortisol determinations. Salivary cortisol levels were determined, in duplicate, by means of a solid phase radioimmuno assay using ${ }^{125} \mathrm{I}$ kits obtained from ICN Biomedicals Inc., CA. The intra- and extra-assay variability for this assay was less than $10 \%$.

\section{Statistical Analysis}

Before conducting tests of the mediation models, correlational analyses between abuse, the mediating variables (coping and social support) and well-being were conducted. The mediation models were assessed using the bootstrap technique. Bootstrapping is a nonparametric procedure wherein a random sample of cases is taken from the total data set, and an estimate of the indirect effect, or point estimate (in this case, the estimate of the magnitude of effectiveness of the mediator) is obtained from that sample. This process is repeated a large number of times (resampling) until confidence intervals for the distribution of the point estimates are generated (Wood, 2005). A significant mediated effect can be concluded if the confidence interval for that effect does not contain zero. Because this method relies on fewer inferential tests than the causal steps approach, it has the advantage of having greater power and lower probability of Type I errors (Preacher \& Hayes, in review). In addition, given the small sample size in the present study, it was considered the most appropriate test to assess mediation.

To assess the ability of coping and social support to mediate the relationship between abuse and well-being, multiple mediators were examined simultaneously, rather than conducting several separate simple mediation analyses. Specifically, the set of coping variables (emotion-focused coping, avoidance, and problem-focused coping) was examined together as a set of potential mediators, while the set of social support variables 
(support from friends, parents and partner) was examined together as a second set. It has been suggested that multiple mediation has several advantages over doing a series of simple mediation tests (Preacher \& Hayes, in review). It allows one to test whether a set of mediators have an overall effect on the relationship between an independent and dependent variable in addition to examining the effects of the individual mediators. As well, it permits the comparison of the relative effectiveness of these mediators as well as assessment of the unique effectiveness of a mediator while controlling for the other mediators included in the model (West \& Aiken, 1997). Finally, including multiple mediators in a model reduces the likelihood of parameter bias, which can occur when a number of hypotheses of indirect effects are tested with a series of simple mediating models (Judd \& Kenny, 1981). The bootstrap technique allows for the assessment of multiple mediators, in contrast to the causal steps method, which may not be appropriate in cases where multiple mediators are involved. For example, in the casual steps approach, it is possible that multiple mediators can produce inconsistent effects, such that the presence of more than one mediator may render the total effect of the independent variable on the dependent variable non-significant (e.g., Judd \& Kenny, 1981).

As per the recommendations of Preacher and Hayes (in review), the program was set up such that 5000 random samples were generated from the original dataset. $95 \%$ confidence intervals for the point estimates (i.e., estimates of the effects in the population that are provided by the sample data) of the indirect effects were generated. It can be concluded that a mediated effect is significant at the .05 level if its confidence interval does not contain zero. Although the program generates several types of confidence intervals, those documented in this report are the bias corrected and accelerated (BCa) 
confidence intervals, which were reported as being highly accurate (see Efron, 1987). In cases where more than one mediator was significant, the results of pairwise contrasts of the indirect effects are reported.

In assessing the effect of stressors on cortisol levels, it is important to note that mildly stressful events may not induce an increase of cortisol, but may attenuate the decline that might otherwise occur when participants are habituating to the laboratory environment. Thus, changes of cortisol levels following the challenge were calculated relative to baseline levels, rather than using absolute values. Hierarchical polynomial regression analyses were conducted in which the three cortisol indices were regressed onto abuse (physical, psychological, or sexual abuse). As the neuroendocrine response to a stressor may vary quadratically with PTSD symptoms (i.e., in an inverted U-shaped fashion, Matheson, 2003), linear PTSD scores were entered on the second step, followed by quadratic (squared) PTSD scores, and finally, the interactions between each type of abuse and each of the two PTSD components.

For the analyses, a $p$ value of .05 or less was used as the criteria for a correlation to be considered statistically significant. $P$ values of less than .10 were also noted as being marginally significant. Since the women in this study were generally in dating relationships, rather than longer married or cohabiting relationships as most previous studies have assessed, it is possible that the correlations found in the present series of studies will not be as strong as those found in a clinical or community sample. However, since abuse escalates over time (e.g., DeKeseredy \& Schwartz, 1998b), it was considered important to acknowledge even smaller correlations. 


\section{Results}

\section{Correlational Analyses}

Correlations between the predictor and outcome variables ${ }^{3}$. To assess the relationship between abuse and symptoms of psychological distress, a multiple regression analysis was conducted in which symptoms were regressed simultaneously onto the three subscales of abuse: psychological aggression, physical assault, and sexual coercion. As indicated in Table 4, abuse significantly predicted current depressive symptoms $\left(R^{2}=.17\right.$, $F(3,139)=9.39, p<.001)$, with each of the three subscales contributing unique variance to current depressive symptomatology. Overall, abuse predicted PTSD symptomatology $\left(R^{2}=.11, F(3,138)=5.92, p<.01\right)$, with sexual and physical abuse contributing unique variance to symptoms.

Table 4. Regression Analysis (Pearson Correlations and Standardized Regression Coefficients) Assessing the Relations between Abuse and Well-Being

\begin{tabular}{|c|c|c|c|c|c|c|}
\hline \multirow[b]{2}{*}{ Abuse } & \multicolumn{3}{|c|}{ Depression } & \multicolumn{3}{|c|}{ PTSD } \\
\hline & $r$ & $\beta$ & $R^{2}$ & $r$ & $\beta$ & $R^{2}$ \\
\hline & & & $7 * * *$ & & & $.11 * *$ \\
\hline Psychological & $.30 * * *$ & $.17^{+}$ & & $.22 * *$ & .10 & \\
\hline Physical & $.32 * * *$ & $.22 *$ & & $.25 * *$ & $.16^{+}$ & \\
\hline Sexual & $.29 * *$ & $.17 *$ & & $.27 * *$ & $.19 *$ & \\
\hline
\end{tabular}

${ }^{+} p<.10 ; * p<.05 ; * * p<.01 ; * * * p<.001$

\footnotetext{
${ }^{3}$ Although the bootstrap procedure does provide the direct effect of an IV on a DV, regression analyses were also performed on these variables in order to report the unique effects of each type of abuse (i.e., when the other types of abuse are controlled for) on symptoms of depression and PTSD.
} 
Correlations among the mediating variables. Correlational analyses were conducted in order to assess the relations among coping and social support. As indicated in Table 5, problem-focused coping was positively associated with support from all three sources. Support from friends and from partner were both positively associated with the use of problem-focused coping, whereas support from parents was negatively related to emotion-focused coping.

Table 5. Correlations between Coping and Social Support

\begin{tabular}{lccc}
\hline & \multicolumn{3}{c}{ Coping } \\
\cline { 2 - 4 } Support & $.31^{* *}$ & -.07 & .12 \\
\cline { 2 - 4 } & Problem-focused & Emotion-focused & Avoidant \\
Friends & .08 & $-.16^{+}$ & .07 \\
Parents & $.21^{*}$ & -.13 & -.11 \\
Partner & & & \\
${ }^{+} p<.10 ; * p<.05 ;{ }^{* *} p<.01$. & &
\end{tabular}

Role of Coping in Mediating the Relationship between Abuse and Well-Being

The first set of analyses examined the potential mediating effects of coping. In addition to the direct effects of abuse on symptoms of mental illness, coping styles might mediate the effects of abuse on such symptoms. Specifically, as mentioned earlier, it was hypothesized that abuse would be associated with the increased use of ineffective coping strategies (i.e., emotion-focused and avoidant), and the decreased use of more adaptive problem-focused strategies, leading to increased vulnerability to developing symptoms of depression and PTSD. 
Analyses of the mediating effects of coping on the relationship between abuse and symptoms of psychological distress were conducted using the bootstrap procedure as described in the preceding sections. First, analyses were performed using depression as the outcome variable, with each of the three types of abuse, in turn, serving as independent variables. Second, analyses were performed with PTSD as the outcome, again with each of the abuse variables as predictors. Although the relationship between the independent and dependent variables is not a necessary precondition for a mediating effect to be present (e.g., Shrout \& Bolger, 2002), it is required that the independent variable be correlated with the potential mediator(s), and with the mediator(s) to be associated with the dependent variable (Collins et al., 1998). In this way, it can be proposed that the independent variable, in this case experience of relationship abuse, was associated with alterations of the mediating variables (i.e., altered coping strategies), which in turn led to a change in the dependent variable (i.e., decreased well-being). Thus, before each mediational analysis, the direct effects of independent variable to mediator, and between mediator to dependent variable, are given, in order to confirm that these relationships are significant before interpreting the results of the bootstrap procedure. The magnitude and direction of the direct effects are given in the output of Preacher and Hayes' (in review) bootstrap program.

Mediation of depression scores by coping. The direct effects of the independent variable on the mediators, and of the mediators on the dependent variable, are presented 
in Table $6^{4}$. All three types of abuse were associated with emotion-focused coping. In addition, both sexual coercion and physical assault were significantly correlated with avoidant coping. In terms of the direct paths between the mediators and dependent variable, both problem-focused and emotion-focused coping were associated with depressive symptoms.

The direct effects of abuse on depression, as well as the indirect effect of abuse on depression (i.e., through its effects on coping) are presented in Table 7. The effects of abuse on BDI scores were reduced when coping was included in the analysis, although the effects were still significant, indicating partial mediation. Three analyses of the indirect effects of coping were conducted. The set of coping scores were entered as the potential mediating variables, depressive symptoms as the dependent variable, and each of the three types of abuse, in turn, as independent variables. As indicated in Table 8, overall, the coping dimensions mediated the effect of psychological aggression on symptoms of depression. However, the only coping dimension for which the confidence interval did not contain zero was emotion-focused coping. Thus, psychological aggression was associated with the increased use of emotion-focused coping, which in turn was associated with symptoms of depression. Similarly, both the overall mediating effect of coping, and the effect of emotion-focused coping in particular, were significant in the sexual coercion-depression association. Finally, although coping did not have an overall mediating effect on the relationship between physical assault and depression,

\footnotetext{
${ }^{4}$ Because bootstrapping is based on taking random samples from a data set, each run of the analysis will produce slightly different estimates of the indirect effect and the standard error, as well as different confidence intervals. Thus, in this table and those that follow, it may be observed that for example, the direct effect of avoidant coping on depression is a different number from the analysis of one potential mediating variable to the next. However, these differences are not large enough that different conclusions would be drawn from varying analyses.
} 
emotion-focused coping did function as a mediator. Thus, emotion-focused coping mediated the effects of abuse, regardless of the type (psychological, sexual, or physical) on symptoms of depression.

Table 6. Direct Effects of Abuse-Coping and Coping-Depression

\begin{tabular}{|c|c|c|c|c|}
\hline & Coefficient & $S E$ & $t$ & $p$ \\
\hline \multicolumn{5}{|l|}{ IV: Psychological Aggression } \\
\hline Psych abuse - Problem-focused & -.01 & .02 & -.73 & .45 \\
\hline Psych abuse - Emotion-focused & .04 & .02 & 2.12 & .04 \\
\hline Psych abuse - Avoidant & .02 & .01 & 1.34 & .18 \\
\hline \multicolumn{5}{|l|}{ Mediator-DV paths } \\
\hline Problem-focused - Depression & -4.27 & .76 & -5.63 & .00 \\
\hline Emotion-focused - Depression & 4.05 & .65 & 6.23 & .00 \\
\hline Avoidant - Depression & 1.16 & .86 & 1.34 & .18 \\
\hline \multicolumn{5}{|l|}{ IV: Sexual Coercion } \\
\hline \multicolumn{5}{|l|}{ IV-Mediator paths } \\
\hline Sexual abuse - Problem-focused & -.08 & .13 & -.61 & .54 \\
\hline Sexual abuse - Emotion-focused & .32 & .16 & 2.05 & .04 \\
\hline Sexual abuse - Avoidant & .25 & .12 & 2.07 & .04 \\
\hline \multicolumn{5}{|l|}{ Mediator - DV paths } \\
\hline Problem-focused - Depression & -4.28 & .76 & -5.60 & .00 \\
\hline Emotion-focused - Depression & 4.14 & .65 & 6.37 & .00 \\
\hline Avoidant - Depression & 1.02 & .88 & 1.17 & .25 \\
\hline \multicolumn{5}{|l|}{ IV: Physical Assault } \\
\hline \multicolumn{5}{|l|}{ IV-Mediator paths } \\
\hline Physical abuse - Problem-focused & .10 & .14 & .76 & .45 \\
\hline Physical abuse - Emotion-focused & .40 & .17 & 2.39 & .02 \\
\hline Physical abuse - Avoidant & .32 & .13 & 2.47 & .01 \\
\hline \multicolumn{5}{|l|}{ Mediator-DV paths } \\
\hline Problem-focused-Depression & -4.47 & .74 & -6.04 & .00 \\
\hline Emotion-focused-Depression & 4.05 & .63 & 6.38 & .00 \\
\hline Avoidant - Depression & .93 & .85 & 1.09 & .28 \\
\hline
\end{tabular}

$S E=$ standard error 
Table 7. Direct and Indirect (through Coping) Effects of Abuse on Symptoms of

Depression: Results of Bootstrap Analysis

\begin{tabular}{|c|c|c|c|c|}
\hline Relationship with BDI Score & Coefficient & $S E$ & $t$ & $p$ \\
\hline \multicolumn{5}{|l|}{ Direct Effects } \\
\hline Psychological abuse & .58 & .15 & 3.79 & .00 \\
\hline Sexual coercion & 4.65 & 1.32 & 3.53 & .00 \\
\hline Physical assault & 5.59 & 1.39 & 4.03 & .00 \\
\hline \multicolumn{5}{|l|}{$\begin{array}{l}\text { Indirect Effects (through } \\
\text { mediator) }\end{array}$} \\
\hline Psychological abuse & .35 & .13 & 2.74 & .01 \\
\hline Sexual coercion & 2.72 & 1.11 & 2.44 & .02 \\
\hline Physical assault & 4.14 & 1.16 & 3.58 & .00 \\
\hline
\end{tabular}


Table 8. Bootstrap Confidence Intervals for the Total and Specific Mediating Effects of Coping on the Abuse-Depression Relationship

\begin{tabular}{|c|c|c|}
\hline & \multicolumn{2}{|c|}{ Bootstrapping 95\% CI } \\
\hline & Lower & Upper \\
\hline \multicolumn{3}{|l|}{ Psychological Aggression } \\
\hline Problem-focused coping & -.11 & .18 \\
\hline Emotion-focused coping & .03 & .32 \\
\hline Avoidant coping & -.00 & .10 \\
\hline TOTAL & .04 & .44 \\
\hline Sexual Coercion & & \\
\hline Problem-focused coping & -.80 & 1.40 \\
\hline Emotion-focused coping & .36 & 2.63 \\
\hline Avoidant coping & -.07 & 1.09 \\
\hline TOTAL & .52 & 3.57 \\
\hline \multicolumn{3}{|l|}{ Physical Assault } \\
\hline Problem-focused coping & -1.83 & .63 \\
\hline Emotion-focused coping & .11 & 3.32 \\
\hline Avoidant coping & -.16 & 1.20 \\
\hline TOTAL & -.31 & 3.34 \\
\hline
\end{tabular}


Mediation of PTSD scores by coping. The direct effects of abuse on coping, and of coping on PTSD, are shown in Table 9. Psychological abuse was associated with emotion-focused coping, while sexual coercion and physical assault were associated with both avoidant and emotion-focused coping styles. Furthermore, both emotion-focused and avoidant coping showed significant correlations with PTSD symptoms.

The direct effects of abuse on PTSD symptoms, and the indirect effect of abuse (when coping was considered as a mediator) are presented in Table 10. All of the effects of abuse were reduced when coping was included in the analysis, although only the effect of psychological aggression became non-significant. Thus, the effects of psychological abuse on PTSD symptoms were completely mediated by coping, whereas the effects of sexual and physical abuse were partially mediated. The mediating effects of the individual coping dimensions are presented in Table 11. Symptoms of PTSD were mediated by overall coping scores for all types of abuse. Specifically, as in the case of depressive symptoms, the association between abuse and PTSD symptoms was mediated by use of emotion-focused coping. As well, both sexual coercion and physical assault were mediated by avoidant coping. Thus, for all forms of abuse, the use of emotionfocused coping was associated with increased vulnerability to PTSD symptoms, whereas for sexual and physical abuse, avoidant coping was also associated with such symptoms. However, as the contrasts in Table 11 indicate, emotion-focused coping and avoidant coping were not significantly different from one another in terms of their ability to mediate the effects of abuse on PTSD symptoms, since the confidence intervals for both contrasts contained zero. 
Table 9. Direct Effects of Abuse-Coping and Coping-PTSD

\begin{tabular}{|c|c|c|c|c|}
\hline & Coefficient & $S E$ & $t$ & $p$ \\
\hline \multicolumn{5}{|l|}{ IV: Psychological Aggression } \\
\hline \multicolumn{5}{|l|}{ IV-Mediator paths } \\
\hline Psych abuse - Problem-focused & -.01 & .01 & -.89 & .37 \\
\hline Psych abuse - Emotion-focused & .04 & .02 & 2.03 & .04 \\
\hline Psych abuse - Avoidant & .02 & .01 & 1.25 & .21 \\
\hline \multicolumn{5}{|l|}{ Mediator-DV paths } \\
\hline Problem-focused - PTSD & -.53 & 2.03 & -.26 & .79 \\
\hline Emotion-focused - PTSD & 9.03 & 1.72 & 5.26 & .00 \\
\hline Avoidant - PTSD & 6.51 & 2.28 & 2.85 & .01 \\
\hline \multicolumn{5}{|l|}{ IV: Sexual Coercion } \\
\hline \multicolumn{5}{|l|}{ IV-Mediator paths } \\
\hline Sexual abuse - Problem-focused & -.09 & .13 & -.73 & .46 \\
\hline Sexual abuse - Emotion-focused & .31 & .16 & 1.99 & .05 \\
\hline Sexual abuse - Avoidant & .24 & .12 & 2.01 & .05 \\
\hline \multicolumn{5}{|l|}{ Mediator - DV paths } \\
\hline Problem-focused - PTSD & -.36 & 2.02 & -.18 & .86 \\
\hline Emotion-focused - PTSD & 9.03 & 1.70 & 5.33 & .00 \\
\hline Avoidant - PTSD & 6.04 & 2.28 & 2.65 & .01 \\
\hline \multicolumn{5}{|l|}{ IV: Physical Assault } \\
\hline \multicolumn{5}{|l|}{ IV-Mediator paths } \\
\hline Physical abuse - Problem-focused & .09 & .13 & .67 & .50 \\
\hline Physical abuse - Emotion-focused & .39 & .17 & 2.34 & .02 \\
\hline Physical abuse - Avoidant & .31 & .13 & 2.42 & .02 \\
\hline \multicolumn{5}{|l|}{ Mediator-DV paths } \\
\hline Problem-focused - PTSD & -.93 & 2.02 & -.46 & .65 \\
\hline Emotion-focused - PTSD & 9.12 & 1.71 & 5.33 & .00 \\
\hline Avoidant - PTSD & 6.25 & 2.30 & 2.72 & .01 \\
\hline
\end{tabular}


Table 10. Direct and Indirect (through Coping) Effects of Abuse on Symptoms of PTSD: Results of Bootstrap Analysis

\begin{tabular}{lcccc}
\hline Relationship with IES Score & Coefficient & $S E$ & $t$ & \\
\hline Direct Effects & & & & \\
$\quad$ Psychological abuse & 1.07 & .40 & 2.68 & .01 \\
Sexual coercion & 11.12 & 3.34 & 3.33 & .00 \\
Physical assault & 5.59 & 1.39 & 4.03 & .00 \\
& & & & \\
Indirect Effects (through & & & & \\
mediator) & .60 & .34 & 1.77 & .08 \\
Psychological abuse & 6.80 & 2.89 & 2.35 & .02 \\
Sexual coercion & 4.14 & 1.16 & 3.58 & .00 \\
Physical assault & & & & \\
\hline
\end{tabular}


Table 11. Bootstrap Confidence Intervals and Contrasts for the Total and Specific

Mediating Effects of Coping on the Abuse-PTSD Relationship

\begin{tabular}{lrr}
\hline & \multicolumn{2}{c}{ Bootstrapping 95\% CI } \\
\hline & Lower & Upper \\
\cline { 2 - 3 } Psychological Aggression & & .17 \\
Problem-focused coping & -.05 & .70 \\
Emotion-focused coping & .08 & .35 \\
Avoidant coping & -.01 & .91 \\
TOTAL & .10 & \\
& & .99 \\
Sexual Coercion & & 5.88 \\
Problem-focused coping & -.50 & 3.83 \\
Emotion-focused coping & .67 & 8.14 \\
Avoidant coping & .19 & \\
TOTAL & 1.14 & 4.46 \\
& & \\
Contrast: Emotion- & -.93 & \\
focused vs. Avoidant & & .37 \\
& & 7.65 \\
Physical Assault & & 4.46 \\
Problem-focused coping & -1.39 & 10.13 \\
Emotion-focused coping & .22 & \\
Avoidant coping & .40 & 5.90 \\
TOTAL & 1.17 & \\
Contrast: Emotion- & -1.74 & \\
focused vs. Avoidant & & \\
\hline
\end{tabular}

Role of Social Support in Mediating the Relationship between Abuse and Well-Being

The role of social support as a mediator in the relationship between abuse and symptoms of depression and PTSD was assessed. As indicated in Figure 1, it was proposed that in addition to its direct effects, abuse may be associated with symptoms of depression and PTSD indirectly, through its effects on social support. That is, abuse may be associated with decreased levels of support from friends, parents, and partner, which may lead to heightened symptoms. 
The direct effects of abuse on the mediators, and of the mediators on the dependent variable, are shown in Table 12. The association between abuse of all types and support from one's partner was significant, although support from friends and parents were not correlated with abuse. Support from one's partner and from friends were also significantly correlated with depression.

As indicated in Table 13, the effects of abuse on symptoms of depression were reduced when the social support variables were considered, although all of the effects remained significant, indicating partial mediation. Analyses of the mediating effects of the specific dimensions of social support on the relationship between abuse and symptoms of psychological distress were conducted. First, analyses were performed using depression as the outcome variable, with each of the three types of abuse in turn as independent variables. As demonstrated in Table 14, overall, the set of social support variables mediated the effects of sexual and physical abuse on symptoms of depression: Moreover, for all types of abuse, support from one's partner mediated the effects of abuse on depression, since the confidence intervals for all three of the indirect effects of partner support did not contain zero. That is, among women experiencing abuse, perceptions of low support from their partner led to increased vulnerability to developing depressive symptoms. 
Table 12. Direct Effects of Abuse-Support and Support-Depression

\begin{tabular}{lccrl}
\hline & Coefficient & \multicolumn{1}{l}{$S E$} & \multicolumn{1}{l}{$t$} & \multicolumn{1}{l}{$p$} \\
\hline IV: Psychological Aggression & & & & \\
IV-Mediator paths & & & & \\
Psych abuse - Friends & .00 & .01 & .35 & .72 \\
Psych abuse - Parents & .01 & .01 & .55 & .58 \\
Psych abuse - Partner & -.01 & .01 & -1.93 & .05 \\
Mediator-DV paths & & & & \\
Friends - Depression & -6.60 & 1.56 & -4.23 & .00 \\
Parents - Depression & -1.58 & 1.00 & -1.58 & .12 \\
Partner - Depression & -5.32 & 1.78 & -2.98 & .00 \\
& & & & \\
IV: Sexual Coercion & & & & \\
IV-Mediator paths & & & & \\
Sexual abuse - Friends & -.09 & .07 & -1.30 & .19 \\
Sexual abuse - Parents & -.11 & .10 & -1.09 & .28 \\
Sexual abuse - Partner & -.13 & .06 & -2.27 & .02 \\
& & & & \\
Mediator - DV paths & & & & \\
Friends - Depression & -5.89 & 1.60 & -3.68 & .00 \\
Parents - Depression & -1.19 & 1.03 & -1.16 & .25 \\
Partner - Depression & -5.90 & 1.82 & -3.23 & .00 \\
& & & & \\
IV: Physical Assault & & & & \\
IV-Mediator paths & & & & .00 \\
Physical abuse - Friends & -.05 & .07 & -.70 & .48 \\
Physical abuse - Parents & -.07 & .11 & -.70 & .49 \\
Physical abuse - Partner & -.17 & .06 & -2.76 & .01 \\
& & & & \\
Mediator-DV paths & -6.22 & 1.59 & -3.91 & .00 \\
Friends - Depression & -1.25 & 1.02 & -1.23 & .22 \\
Parents - Depression & -5.42 & 1.84 & -2.95 & .00 \\
Partner - Depression & & & &
\end{tabular}


Table 13. Direct and Indirect (through Social Support) Effects of Abuse on Symptoms of Depression: Results of Bootstrap Analysis

\begin{tabular}{lcccc}
\hline Relationship with IES Score & Coefficient & \multicolumn{2}{l}{$t$} & \multicolumn{2}{l}{$p$} \\
\hline Direct Effects & & & & \\
$\quad$ Psychological abuse & .58 & .15 & 3.82 & .00 \\
$\quad$ Sexual coercion & 4.75 & 1.31 & 3.63 & .00 \\
$\quad$ Physical assault & 5.36 & 1.41 & 3.79 & .00 \\
& & & & \\
Indirect Effects (through & & & & \\
mediator) & .54 & .13 & 4.06 & .00 \\
$\quad$ Psychological abuse & 3.33 & 1.17 & 2.84 & .01 \\
$\quad$ Sexual coercion & 4.01 & 1.27 & 3.15 & .00 \\
\hline Physical assault & & & & \\
\hline
\end{tabular}


Table 14. Bootstrap Confidence Intervals for the Total and Specific Mediating Effects of Social Support on the Abuse-Depression Relationship

\begin{tabular}{|c|c|c|}
\hline & Bootstrapp & $95 \% \mathrm{CI}$ \\
\hline & Lower & Upper \\
\hline \multicolumn{3}{|c|}{ Psychological Aggression } \\
\hline Friends & -.10 & .08 \\
\hline Parents & -.05 & .02 \\
\hline Partner & .00 & .23 \\
\hline TOTAL & -.10 & .23 \\
\hline \multicolumn{3}{|c|}{ Sexual Coercion } \\
\hline Friends & -.18 & 1.82 \\
\hline Parents & -.08 & .89 \\
\hline Partner & .07 & 2.27 \\
\hline TOTAL & .06 & 3.20 \\
\hline \multicolumn{3}{|c|}{ Physical Assault } \\
\hline Friends & -.31 & 1.76 \\
\hline Parents & -.10 & .84 \\
\hline Partner & .19 & 2.48 \\
\hline TOTAL & .15 & 3.29 \\
\hline
\end{tabular}

Mediation of PTSD scores by social support. As indicated in Table 15, although partner support was associated with abuse, support was unrelated to PTSD symptoms. Since the relationship between the mediator and outcome variable must be significant in order for a mediating effect to be present (Shrout \& Bolger, 2002), the indirect effects of social support were not assessed further. 
Table 15. Direct Effects of Abuse - Social Support and Social Support - PTSD

\begin{tabular}{|c|c|c|c|c|}
\hline & Coefficient & $S E$ & $t$ & $p$ \\
\hline \multicolumn{5}{|l|}{ IV: Psychological Aggression } \\
\hline \multicolumn{5}{|l|}{ IV-Mediator paths } \\
\hline Psych abuse - Friends & .00 & .01 & .26 & .80 \\
\hline Psych abuse - Parents & .01 & .01 & .58 & .56 \\
\hline Psych abuse - Partner & -.01 & .01 & -1.97 & .05 \\
\hline \multicolumn{5}{|l|}{ Mediator-DV paths } \\
\hline Friends - PTSD & 4.89 & 4.74 & 1.03 & .30 \\
\hline Parents - PTSD & -3.93 & 3.00 & -1.31 & .19 \\
\hline Partner - PTSD & -3.87 & 5.36 & -.72 & .47 \\
\hline \multicolumn{5}{|l|}{ IV: Sexual Coercion } \\
\hline \multicolumn{5}{|l|}{ IV-Mediator paths } \\
\hline Sexual abuse - Friends & -.09 & .13 & -.73 & .46 \\
\hline Sexual abuse - Parents & .31 & .16 & 1.99 & .05 \\
\hline Sexual abuse - Partner & .24 & .12 & 2.01 & .05 \\
\hline \multicolumn{5}{|l|}{ Mediator - DV paths } \\
\hline Friends - PTSD & 6.43 & 4.65 & 1.38 & .17 \\
\hline Parents - PTSD & -2.91 & 2.96 & -.99 & .33 \\
\hline Partner - PTSD & -3.78 & 5.24 & -.72 & .47 \\
\hline \multicolumn{5}{|l|}{ IV: Physical Assault } \\
\hline \multicolumn{5}{|l|}{ IV-Mediator paths } \\
\hline Physical abuse - Friends & -.06 & .07 & -.77 & .44 \\
\hline Physical abuse - Parents & -.07 & .11 & -.68 & .50 \\
\hline Physical abuse - Partner & -.17 & .06 & -2.78 & .01 \\
\hline \multicolumn{5}{|l|}{ Mediator-DV paths } \\
\hline Friends - PTSD & 5.50 & 4.73 & 1.16 & .25 \\
\hline Parents - PTSD & -3.23 & 3.00 & -1.08 & .28 \\
\hline Partner - PTSD & -3.52 & 5.40 & -.65 & .52 \\
\hline
\end{tabular}


Summary of mediating relationships. In summary, the use of ineffective coping strategies, and perceptions of low social support from one's partner, mediated the effects of abuse on well-being. Specifically, the association between abuse and depression, and that between abuse and PTSD symptoms, was mediated by the use of emotion-focused coping. As well, avoidant coping was a mediator in the abuse-PTSD relationship. Support from one's partner mediated the effects of abuse on symptoms of depression. When alternative mediation models were tested, using mental health symptoms as the independent variable and abuse as the dependent variable, these were not found to be significant (data not shown), indicating that coping and social support were mediators of the impact of abuse on psychological distress, and not vice versa.

\section{Neuroendocrine Response Elicited by a Challenge}

Since anti-inflammatory and antidepressant medications may affect HPA functioning (e.g., Calfa, Kademian, Ceschin, Vega, Rabinovitch, et al., 2003; Knutsson, Stierna, Marcus, Carlstedt-Duke, Carlstrom, et al., 1995), the analyses involving cortisol were run both with and without the individuals taking these medications. However, since the results did not differ as a function of whether these data were included, participants on such medication were left in the analyses reported. Since it had previously been shown that oral contraceptives can induce hormonal effects that can impact cortisol levels (e.g., Kirschbaum, Kudielka, Gaab, Schommer, \& Hellhammer, 1999; Reinberg, Touitou, Soudant, Bernard, Bazin, \& Mechkouri, 1996), use of this medication was controlled for in the analyses. That is, for all of the regression analyses, a variable indicating reported use of oral contraceptives was entered as the first step before any of the variables of interest. To assess the association between abuse and baseline cortisol levels, a regression 
analysis was conducted in which the three types of abuse were regressed against baseline cortisol. Table 16 presents the mean levels of cortisol at baseline and in response to the challenge.

Table 16. Cortisol Levels ( $\mu \mathrm{g} / \mathrm{dl}$ ) as a Function of Abuse Type

\begin{tabular}{lcccccc}
\hline & \multicolumn{2}{c}{ Baseline } & \multicolumn{2}{c}{$\begin{array}{c}\text { 15 min after } \\
\text { challenge }\end{array}$} & \multicolumn{2}{c}{$\begin{array}{c}\text { min after } \\
\text { challenge }\end{array}$} \\
\hline Mbuse type & Mean & SE & Mean & SE & Mean & SE \\
$\quad$ Psychological & .99 & .10 & 1.02 & .09 & 1.00 & .09 \\
Physical & .89 & .12 & .86 & .11 & .86 & .11 \\
Sexual & .93 & .11 & .97 & .11 & .96 & .11 \\
& & & & & & \\
$\quad$ No abuse & .88 & .06 & .90 & .06 & .84 & .06 \\
\hline
\end{tabular}

A regression analysis indicated that abused and non-abused women could not be distinguished on the basis of baseline cortisol levels (Table 17).

Table 17. Relationship of Abuse to Baseline Cortisol Levels

\begin{tabular}{lccc}
\hline & $r$ & $\beta$ & $R^{2}$ \\
\hline Abuse type & & & .03 \\
Psychological & .09 & .13 & \\
Physical & -.06 & -.08 & \\
Sexual & -.07 & -.10 & \\
\hline
\end{tabular}

Next, to assess the relationship between abuse and the cortisol response to the stressor as it related to PTSD symptoms, hierarchical polynomial regression analyses 
were conducted on cortisol ratios following the challenge. Following the use of oral contraceptives, abuse was entered on the first step, followed by IES score, IES (quadratic) score, and the interaction terms (abuse*IES, abuse*IES [quadratic]) ${ }^{5}$. Current involvement in an abusive relationship, regardless of type of abuse, did not moderate women's cortisol reactions to their reports of past trauma (see Tables 18-20). In addition, PTSD symptoms were not significantly related to cortisol change in response to the stressor. Neither of the interaction terms was significant. Thus, the neuroendocrine response to the stressor challenge did not vary either linearly or quadratically with PTSD symptoms, nor did PTSD symptoms and abuse interact to produce a significant alteration in cortisol levels.

\footnotetext{
${ }^{5}$ To compute the interaction terms in the analyses, standardized (z) scores were obtained for the continuous variables, psychological abuse and PTSD, by subtracting each variable's mean from the individual cases and then dividing by the standard deviation. The cross-products were then computed using these standardized scores in order to examine the interactive effects of abuse and PTSD. The dichotomous variables (sexual and physical abuse) and quadratic PTSD scores were not standardized. Standardization of scores of continuous predictor or moderator variables reduces problems associated with multicollinearity, or high correlations among variables in the regression equation (Garson, n.d.).
} 
Table 18. Regression Coefficients Predicting Cortisol Ratios as a Function of Psychological Abuse, PTSD Symptoms and Mild Challenge

\begin{tabular}{|c|c|c|c|}
\hline & $\bar{r}$ & $\beta^{6}$ & $R_{\text {change }}^{2}$ \\
\hline \multicolumn{4}{|l|}{ Ratio of $15 \mathrm{~min}$ post-challenge/baseline } \\
\hline Psychological abuse & -.00 & .00 & .00 \\
\hline PTSD symptoms & -.03 & .02 & .00 \\
\hline PTSD symptoms (quadratic) & .00 & .38 & .01 \\
\hline Interactions between psych. abuse \& & & & .01 \\
\hline PTSD symptoms & .14 & -.19 & \\
\hline PTSD symptoms (quadratic) & .19 & .52 & \\
\hline \multicolumn{4}{|l|}{ Ratio of 30 min post-challenge/baseline } \\
\hline Psychological abuse & -.13 & -.12 & .02 \\
\hline PTSD symptoms & -.11 & -.05 & .00 \\
\hline PTSD symptoms (quadratic) & -.10 & .10 & .00 \\
\hline Interactions between psych. abuse \& & & & .01 \\
\hline PTSD symptoms & .11 & .19 & \\
\hline PTSD symptoms (quadratic) & -.04 & -.12 & \\
\hline
\end{tabular}

\footnotetext{
${ }^{6}$ In Tables 18-20, while the proportion of variance explained is the contribution made on each step of the hierarchical regression, the regression coefficients represent final step weights.
} 
Table 19. Regression Coefficients Predicting Cortisol Ratios as a Function of Sexual Abuse, PTSD Symptoms and Mild Challenge

\begin{tabular}{lrrr}
\hline & $r$ & $\beta$ & $R_{\text {change }}^{2}$ \\
\hline Ratio of 15 min post-challenge/baseline & & & \\
Sexual abuse & .11 & .15 & .02 \\
PTSD symptoms & -.03 & -.02 & .00 \\
PTSD symptoms (quadratic) & .00 & .36 & .01 \\
Interactions between sexual abuse \& & & & .02 \\
$\quad$ PTSD symptoms & .10 & -1.20 & \\
$\quad$ PTSD symptoms (quadratic) & .11 & .98 & \\
Ratio of 30 min post-challenge/baseline & & & \\
Sexual abuse & .04 & .06 & .00 \\
PTSD symptoms & -.11 & -.09 & .01 \\
PTSD symptoms (quadratic) & -.10 & .10 & .00 \\
Interactions between sexual abuse \& & & & .01 \\
$\quad$ PTSD symptoms & .04 & -.09 & \\
$\quad$ PTSD symptoms (quadratic) & .04 & .31 & \\
\hline
\end{tabular}


Table 20. Regression Coefficients Predicting Cortisol Ratios as a Function of Physical Abuse, PTSD Symptoms and Mild Challenge

\begin{tabular}{lccr}
\hline & $r$ & $\beta$ & $R^{2}$ change \\
\hline Ratio of 15 min post-challenge/baseline & -.03 & -.02 & .00 \\
Physical abuse & -.03 & .02 & .00 \\
PTSD symptoms & .00 & .38 & .01 \\
PTSD symptoms (quadratic) & & & .01 \\
Interactions between physical abuse \& & .01 & .21 & \\
$\quad$ PTSD symptoms & .03 & .09 & \\
$\quad$ PTSD symptoms (quadratic) & & & \\
Ratio of 30 min post-challenge/baseline & -.10 & -.08 & .01 \\
Physical abuse & -.11 & -.05 & .00 \\
PTSD symptoms & -.09 & .10 & .00 \\
PTSD symptoms (quadratic) & & & .02 \\
Interactions between physical abuse \& & -.05 & 1.22 & \\
$\quad$ PTSD symptoms & -.03 & -.55 & \\
$\quad$ PTSD symptoms (quadratic) & & & \\
\hline
\end{tabular}

Discussion

As in the case of severe abuse (Golding, 1999; Nixon, Resick, \& Nishith, 2004; Stein \& Kennedy, 2001), among women in abusive dating relationships, symptoms of depression were higher than among women who had not encountered such experiences. Certainly, the severity of the depressive symptoms was, in the main, at subclinical levels; however, in a subset of the abused women the symptom severity was sufficient to be of concern, particularly as subsyndromal depression may be a harbinger of more severe 
illness (Angst \& Merikangas, 1997; Broadhead, Blazer, George, \& Tse, 1990; Judd, Akiskal, \& Paulus, 1997).

In line with the findings regarding depressive symptoms, women in abusive relationships also exhibited heightened levels of PTSD, although only sexual coercion emerged as a significant unique predictor, while physical abuse exhibited a marginally significant unique correlation with PTSD. Psychological aggression, in contrast, was not uniquely associated with symptoms when the other types of abuse were considered. However, others (e.g., Taft et al., 2005) reported that psychological abuse is a predictor of PTSD above and beyond the effects of physical violence. Differences between the results of the present study and those of earlier reports may stem from the disparate populations being studied. For example, although Pico-Alfonso (2005) found that all types of abuse contributed significantly and uniquely to PTSD symptomatology, individuals in that study were recruited from an outpatient counselling agency for battered women, and physical abuse was a criterion for participating in the study. Finally, given that the magnitude of the correlations of PTSD to psychological aggression and physical assault were similar to that between PTSD and sexual abuse (which was significant), it may be premature to conclude that the various types of abuse do not contribute individually to symptoms.

The effects of abuse on symptoms of depression and PTSD appeared to be mediated by the coping strategies used to deal with conflict. Experiences of psychological, physical or sexual abuse may be associated with the increased use of ineffective styles of coping with relationship events, which may in turn lead to the emergence of mental health symptoms. Indeed, such coping strategies have previously 
been shown to relate to symptoms of depression and PTSD (e.g., Arias \& Pape, 1999; Clements \& Sawhney, 2000), although most such studies examined battered women in the later stages of such relationships. The finding that abuse, ineffective coping styles, and symptoms of mental illness occur at such an early stage of dating points to the need to target younger women in the implementation of educational and intervention strategies, particularly since negative patterns of interaction may be more likely to change before escalation of abuse occurs (Saltzman et al., 2000). Of course, it is recognized that the present study as well as previous reports are correlational, and thus the causal nature or directionality of relationships between abuse, coping, and mental health symptoms can only be speculated.

Coping may be influenced by contextual characteristics related to the situation (e.g., Waldrop \& Resick, 2004). Thus, it would not be surprising to see that coping might vary across different types of conflicts within relationships (e.g., conflicts regarding financial matters, versus those pertaining to family or friends). In the present study, participants were asked to pick one conflict with their partner, and report the coping strategies used to deal with this event. Therefore, the results of the present study cannot be generalized across situations, since it is possible that the coping strategies used in response to the event chosen are not necessarily reflective of general patterns of coping with adverse events, or even with various conflicts within a relationship. Furthermore, this approach assumes that all participants choose equally significant or aversive situations, when in fact some participants may report an incident of severe abuse while others may choose a relatively benign conflict. 
Finally, as predicted, abused women reported less social support than did nonabused women, and this was particularly notable with respect to both physically and psychologically abused women. Furthermore, partner support mediated the effects of abuse on symptoms of depression. Perhaps not unsurprisingly, women in abusive relationships may perceive their partners as not being supportive as a result of the abuse. Perceived lack of support may, in turn, lead to decreased well-being. This finding is in accordance with that of Carlson et al. (2002), who found that abuse was related to lack of partner support. In contrast, others found that abuse was, indeed, associated with lack of support from sources other than the partner (e.g., Pipes \& LeBov-Keeler, 1997). Differences may exist, however, in the sampling methods used. University women may be more likely to be surrounded by a supportive network of friends, compared to battered women, who may be isolated as a result of fear or humiliation of the consequences of reporting abuse, or due to forced isolation by the partner. As well, variant findings may be a result of the method of assessing support. In this regard, it was found that abuserelated PTSD was associated with the amount of interpersonal friction, rather than the degree of positive support per se (which is what most studies aim to measure) (Zoellner, Foa, \& Brigidi, 1999). In addition to its relationship with abuse, support from friends and partner was associated with the increased use of problem-focused coping, which is in line with previous research that demonstrated the positive effects of social support on coping efforts (Thoits, 1986).

Levels of cortisol at baseline, and following a challenge, did not vary as a function of abuse or PTSD symptoms. It is possible that the lack of significant results occurred because participants were not sufficiently engaged in the challenge. Although 
others have found that experiences of abuse are related to cortisol alterations (e.g., PicoAlfonso et al., 2004), such studies have generally used women from domestic violence help centers, who had likely been experiencing abuse for a prolonged period of time. Among these women, the experience of severe and protracted stressors may have caused enduring changes of neuroendocrine functioning. It may be more difficult to detect alterations of hormone levels among women in abusive dating relationships. As well, it has previously been found that if women were "primed" to recall their stressor history before watching a video depicting an abusive situation, the video was associated with exceptionally high cortisol elevations among women with high levels of PTSD (Matheson, 2003). In the present investigation, however, women did not complete a questionnaire prior to viewing the photographs and hence a priming stimulus was not present. Thus, it is possible that marked elevations in cortisol following a stressor would not be evident unless women were primed to recall past stressful events. This possible shortcoming was addressed in the second study.

\section{Study 2}

Study 1 revealed that women in abusive relationships may use ineffective means of coping with stressful circumstances, which could be associated with symptoms of psychological distress. In particular, it was found that the endorsement of emotionfocused or avoidant coping strategies was associated with the presence of symptoms characterizing depression and PTSD. However, it is unclear whether abused women use more adaptive, effective emotion-focused styles of coping less frequently than those not in abusive relationships. It has been suggested that in certain circumstances emotional approach, a more adaptive form of emotional coping that involves the use of emotions to 
actively deal with stressors, may be associated with lower anxiety and depressive symptoms, and greater life satisfaction and self-esteem (Stanton et al., 1994; Stanton et al., 2000a). Thus, to the extent that women in abusive relationships utilize emotional approach strategies, they should be able to buffer the effects of stressful experiences accordingly. Given the differentiation between different types of emotional coping, in addition to assessing coping strategies categorized along the lines of emotion-focused, problem-focused, and avoidant, in the present study, the use of emotional approach coping was also assessed as a potential mediating variable in the association between abuse and symptoms of psychological distress. It is possible that the use of active, emotional approach coping strategies, including the expression and processing of emotions, may mitigate the negative effects of abusive experiences on symptoms of depression and PTSD. Furthermore, the ability of emotional approach coping (i.e., emotional processing and emotional expression) to mediate symptoms of depression and PTSD related to abuse was compared to that of emotion-focused, avoidant, and problemfocused strategies.

Study 2 sought to determine whether women in abusive relationships exhibited altered appraisals of relationship conflicts. In order to address this, participants responded to a series of questions regarding relationship conflicts and potentially abusive behaviours. It was hypothesized that appraisals would mediate the relation between abusive experiences and symptoms of depression and PTSD. In effect, if women do not perceive abusive behaviours as such, then they will be less likely to develop symptoms of psychological distress. However, in addition to assessing the separate mediating effects of coping and appraisals, appraisals were also examined for their role as potentially distal 
mediators of depression and PTSD. Appraisals may impact upon psychological distress indirectly: perceptions of a stressor may influence the coping strategy used, which may in turn lead to psychological distress. It is thought that appraisals and coping methods work in concert to determine reactions to stressors. That is, appraisals of events (e.g., perceived threat or challenge) may affect the coping strategies endorsed (Folkman \& Lazarus, 1980). It follows that women in abusive relationships may be unable to invoke appropriate coping strategies, as the perceptions or appraisals of their situation and their emotional reactions to it may be impaired. Therefore, the role of appraisals in mediating the relationship between abuse and coping strategies was tested. It was hypothesized that women who exhibit maladaptive appraisals of potentially abusive events (e.g., not perceiving malevolent behaviours as instances of abuse) would be more likely to invoke ineffective coping strategies.

Although abuse was associated with PTSD in Study 1, as in previous studies (e.g., Coker et al., 2002), women were asked to report on any recent or past stressful event when answering the IES. Although some participants may have reported a severe stressor (e.g., car accident, previous sexual assault), others may have considered a relatively benign experience (e.g., failed an exam, got a parking ticket). In Study 2, it was decided that PTSD would be assessed in relation to a stressful event related to a relationship conflict. Thus, the instructions for the Impact of Events Scale were changed for this study such that participants were asked to respond to the items that pertained to a recent abusive encounter, or a recent conflict with their partner (if they had not experienced abuse). The mediational model assessed in this study is depicted in Figure 2. 


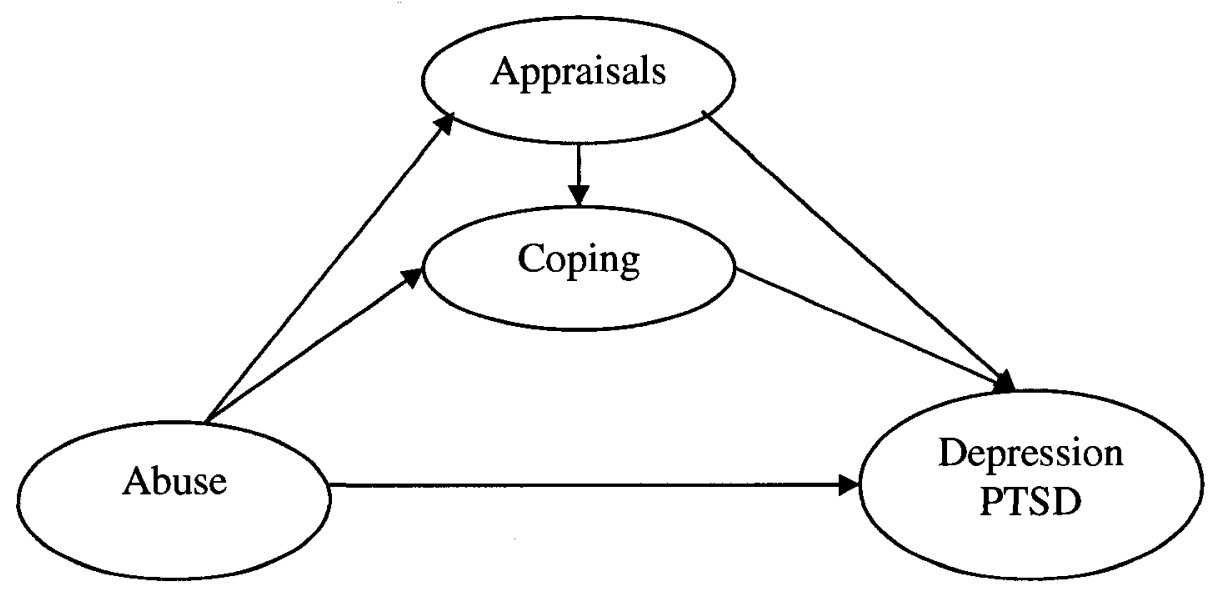

Figure 2. Mediating Effects of Coping and Appraisals on the Relationship Between Abuse and Symptoms of Depression and PTSD

Finally, baseline cortisol levels, as well as cortisol responses to a mild challenge, were assessed in Study 2. Although Study 1 evaluated the neuroendocrine response to a stressor, the relative effects of processing information about the different pictures could not be determined. That is, it was not possible to disentangle the effects of cortisol release following the processing of information regarding relationship abuse, versus processing of the other stressful scenes that did not depict abusive behaviours. To address this issue, the present study assessed neuroendocrine activity following the viewing of a single picture depicting relationship abuse, a stressful but non-abusive scene, or a neutral scene, with participants being divided into three groups according to the photograph they were shown. As well, Study 2 involved more active engagement in the challenge, in that participants were asked to speak about the photograph in front of a video camera. As mentioned earlier, it was found that if women were "primed" to recall stressful experiences prior to watching a video depicting abuse, they were more likely to exhibit 
heightened levels of cortisol (Matheson, 2003). Thus, in this study women were primed to recall past events prior to viewing the photographs. To do this, the participants were asked to complete a number of questionnaires regarding past experiences of abuse and other stressors before the challenge was given, thus making these past experiences salient.

\section{Methods}

\section{Participants}

First year university women from an undergraduate psychology course $(N=118$; $M$ age $=19.96, S D=2.52)$ were recruited, through mass testing and sign-up sheets (Appendix 1), to participate in a study on heterosexual dating relationships. The majority $(n=76,64.4 \%)$ were in their first year of studies. The others were in second year $(n=21$, $17.8 \%)$, third year $(n=9,7.6 \%)$ or fourth year $(n=8,6.8 \%)$, or did not report year of study $(n=4,3.4 \%)$. Most participants reported being on some form of prescription medication: birth control $(n=68,58.1 \%)$, anti-inflammatories $(n=3,2.5 \%)$, antidepressants $(n=1, .8 \%)$, anxiolytics $n=1, .8 \%)$, or other medications $(n=12$, $10.2 \%)$. As well, most participants had never been in therapy $(n=90,76.3 \%)$, whereas 26 participants $(22.0 \%)$ had been in therapy but were no longer, and two $(1.7 \%)$ were in therapy at the time of testing.

On average, women reported having been in their relationship for 18.71 months $(S D=13.32 ;$ Range $=.50$ to 48 months $)$, and time in the relationship did not differ between abusive and non-abusive relationships. Of the women who reported ethnic status, the majority ( $n=85,72.0 \%$ ) were Euro-Caucasian. Others were Middle Eastern ( $n$ $=6,5.1 \%)$, Black $(n=4,3.4 \%)$, Asian $(n=13,11.0 \%)$, Hispanic $(n=3,2.5 \%)$, Native 
Canadian $(n=2,1.7 \%)$, or of mixed racial background $(n=1, .8 \%)$.

As in Study 1, women completed the TLEQ (Kubany et al., 2000) in light of the possibility that past experiences of trauma and abuse may impact current well-being. As indicated in Table 21, women in abusive relationships did not differ from non-abused women with respect to the frequency of experiencing a shocking or unexpected event, an unexpected death, witnessing of a negative event, or assault.

Table 21. Frequencies (and Percentages) of Previous Traumatic Experiences among Abused versus Non-Abused Women

\begin{tabular}{lcc}
\hline & Abused $(\mathrm{N}=86)$ & Non-abused (N =32) \\
\hline Shocking/unexpected event & $48(55.8 \%)$ & $20(62.5 \%)$ \\
Unexpected death & $45(52.3 \%)$ & $17(53.1 \%)$ \\
Witness negative event & $43(50.0 \%)$ & $17(53.1 \%)$ \\
Assault & $51(59.3 \%)$ & $18(56.3 \%)$ \\
\hline
\end{tabular}

\section{Procedure}

Women completed an informed consent form as well as measures assessing the current status of their relationship, abuse, coping, appraisals, and psychological wellbeing in terms of symptoms of depression and PTSD. In addition, they were randomly assigned to be exposed to either the abuse picture $(n=43)$, the accident scene $(n=35)$ or the neutral picture $(n=40)$. Saliva samples were taken at three times, namely $15 \mathrm{~min}$ after entering the laboratory, as well as $15 \mathrm{~min}$ and $30 \mathrm{~min}$ following the challenge. Upon completion of the studies, the participants were debriefed and provided with contact information in the event that they wished to seek help associated with problematic 
relationships or distress symptoms (Appendix 2). All procedures were approved by the Ethics committee of Carleton University and met the Canadian tri-council guidelines.

\section{Measures}

Abuse. Experiences of violence in a relationship were measured by the 39 -item Conflict Tactics Scale-Revised (CTS-R), as described in Study 1 (Straus et al., 1996). Scale reliability analyses indicated that reliability was high for the psychological aggression subscale $(\alpha=.73)$. As in Study 1 , reliability of the sexual coercion subscale was relatively low $(\alpha=.60)$. Two of the items considered 'severe' by Straus et al. (1996) were not endorsed by any participant, while the other two severe items were each only endorsed by two participants. When the two items not endorsed were removed from the analysis, reliability increased to .64 . The physical assault subscale was highly reliable ( $\alpha$ $=.77)$, whereas the injury subscale reliability was low $(\alpha=.51)$. Only 4 items in the injury scale had actually been endorsed. However, people who had also indicated physical assault endorsed all these. Thus, the injury scale was not used in the study since all of the participants indicating injury were already captured in the physical assault subscale. As in Study 1, participants tended to report experiencing milder physically abusive behaviours rather than those of a severe nature.

As in Study 1, the physical and sexual abuse variables were highly skewed (sk $=4.33$ and 3.04, respectively). Psychological aggression was less skewed, with a value of 1.00 $(\mathrm{SE}=.22)$. To be consistent with Study 1 , the sexual and physical abuse variables were dichotomized, such that women were categorized as being in a physically or sexually abusive relationship if they indicated having experienced any physical abuse/injury or 
sexual abuse, respectively (i.e., a score of 1 or greater). Psychological abuse was left as a continuous variable.

The final sample of women comprised 86 women who were in abusive relationships and 32 who were in non-abusive relationships. Specifically, 30 participants $(25.4 \%)$ experienced physical abuse/injury, and $42(35.6 \%)$ experienced sexual coercion. As well, $59(50.0 \%)$ reported psychological aggression scores of 3 or greater. Forty $(33.9 \%)$ of the women reported multiple forms of abuse. Psychological abuse was significantly associated with both physical abuse $(r=.48, p<.001)$ and sexual coercion $(r=.39, p<$ $.001)$. Physical and sexual maltreatment were not correlated $(r=.12, n s)$. As in Study 1 , the variability of scores reflecting sexual and physical abuse in dating relationships was low. Overall, the abuse reported was relatively low (physical assault $M=.90 /$ month, $S D$ $=2.47$; sexual coercion $M=1.26 /$ month, $S D=2.55)$. Psychological aggression scores were higher and more variable $(M=4.03, S D=4.19)$.

Depressive symptoms. The 21-item version of the Beck Depression Inventory (BDI, Beck \& Beck, 1972) was used as an indicator of depressive symptoms. The scale was highly reliable $(\alpha=.85)$. Scores on the BDI ranged between 0 and $37(M=10.64 ; S D=$ $6.94)$.

Symptoms of posttraumatic stress disorder. The 22-item Impact of Events Scale (IESR; Weiss \& Marmar, 1997) was used to assess PTSD symptoms. The scale was highly reliable $(\alpha=.93)$. Scores on the IES ranged between 0 and $71(M=24.00 ; S D=16.67)$.

Coping styles. The 50-item Survey of Coping Profile Endorsement (SCOPE; Matheson \& Anisman, 2003) was used to assess the strategies utilized by women to deal with conflict within an intimate relationship. To be consistent with Study 1, the coping 
scale was subdivided into problem-focused coping (containing problem solving, cognitive restructuring, active distraction, humour, and social support; $\alpha=.68$ ), emotionfocused coping (containing rumination, self-blame, wishful thinking, and emotional expression; $\alpha=.84$ ), and avoidant coping (containing avoidance, passivity, emotional containment, and other blame; $\alpha=.67$ ). The correlations among the 14 subscales and the 3 overall coping style dimensions are presented in Table 22 and Table 23, respectively. As in Study 1, the avoidant and problem-focused subscales, as well as the avoidant and emotion-focused subscales, were significantly correlated. However, in this study, problem-focused coping was not associated with emotion-focused coping. 


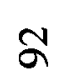

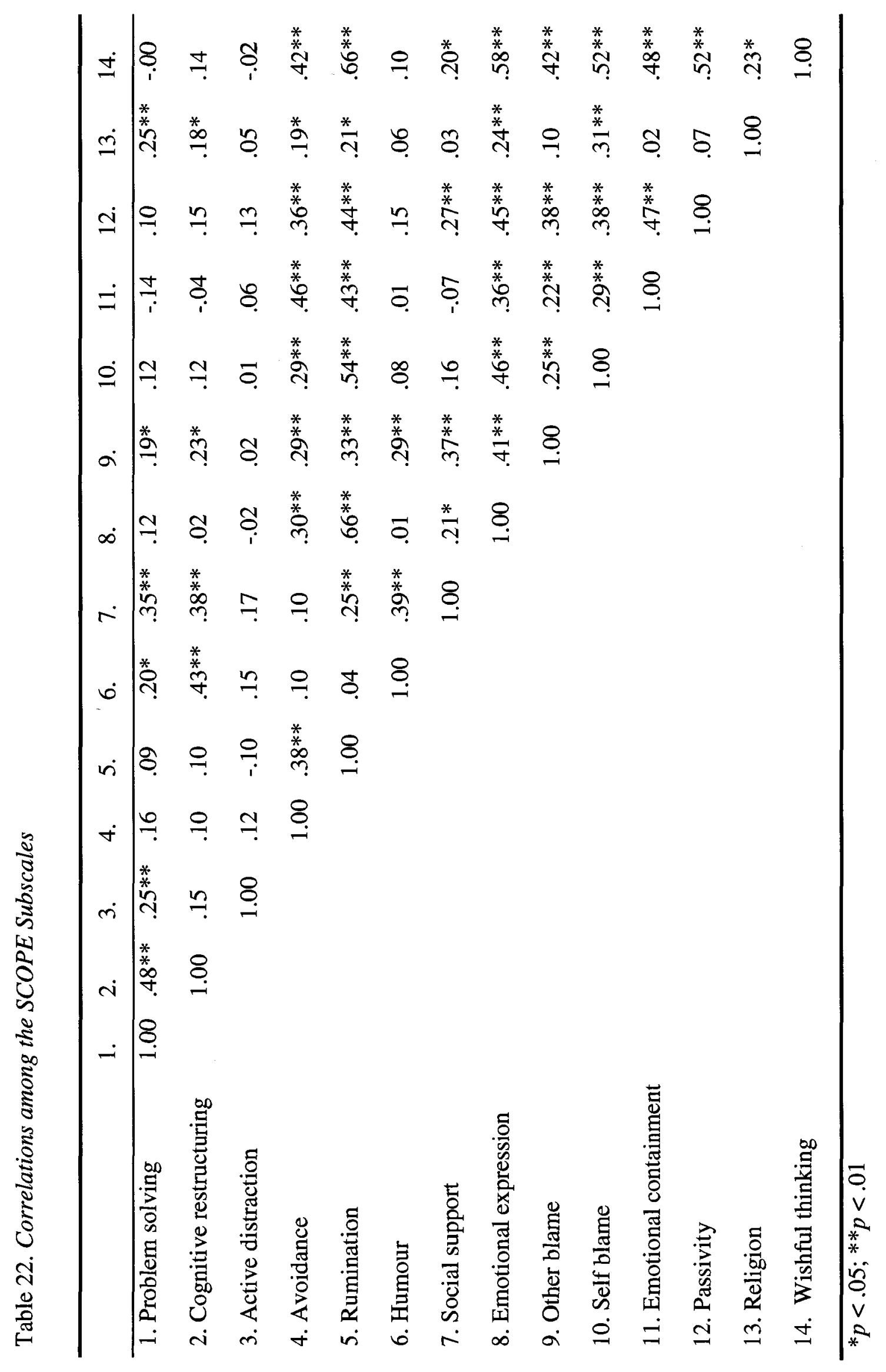


Table 23. Correlations among the Dimensions of Coping

\begin{tabular}{|c|c|c|c|}
\hline & 1. Problem-focused & 2. Emotion-focused & 3. Avoidant \\
\hline & 1.00 & .15 & $.24^{*}$ \\
\hline 2. & & 1.00 & $.66^{* * *}$ \\
\hline 3. & & & 1.00 \\
\hline
\end{tabular}

Emotional coping. The Emotional Coping Scale (ECS; Stanton et al., 2000b) was used to assess the use of emotional approach coping. The scale consists of two subscales, each containing 4 items, which represent two dimensions of emotional approach coping: emotional processing (i.e., active attempts to acknowledge and comprehend one's emotions) and emotional expression. In a previous study, the scale was related to problem-focused coping indices and uncorrelated with avoidant coping, and negatively related to depression and anxiety (Stanton et al., 2000b). The reliability of the processing subscale was .68 , and that of the emotional expression subscale was .86 . The dimensions of the ECS were not related to the emotion-focused dimensions of the SCOPE, as indicated in Table 24. However, emotional expression was inversely related to the emotional containment dimension of avoidant coping. As well, both of the subscales of the Emotional Coping Scale were positively correlated with the problem-solving and cognitive restructuring dimensions of problem-focused coping from the SCOPE. 
Table 24. Correlations among the Dimensions of the ECS and the SCOPE

\begin{tabular}{lcc}
\hline & \multicolumn{2}{c}{ Dimensions of Emotional Approach Coping } \\
\hline & Emotional Processing & Emotional Expression \\
\cline { 2 - 3 } Dimensions of Emotion- & & \\
Focused Coping (SCOPE) & & -.07 \\
Rumination & .06 & .02 \\
Emotional expression & .13 & .02 \\
Self-blame & -.05 & -.01 \\
Wishful thinking & -.01 & \\
& & \\
Dimensions of Avoidant & & -.10 \\
Coping (SCOPE) & & -.14 \\
Avoidance & -.07 & $-.23^{*}$ \\
Passivity & -.14 & .07 \\
Emotional containment & -.07 & \\
Other blame & .10 & .10 \\
& & .06 \\
Dimensions of Problem- & & $.27^{* *}$ \\
Focused Coping (SCOPE) & .13 & \\
Problem-solving & $.36^{* *}$ & .06 \\
Cognitive restructuring & $.30^{* *}$ & \\
Active distraction & .04 & \\
Humour & .12 & \\
Social support & .15 & \\
\hline
\end{tabular}


Appraisals. Appraisals were assessed using two methods. First, to assess participants' perceptions of abusive behaviours, the CTS-R was given a second time, but the instructions were altered such that participants were asked to indicate to what extent they considered each behaviour to be abusive, from a scale of 0 (strongly disagree that behaviour is abusive) to 4 (strongly agree that behaviour is abusive). This version of the scale was highly reliable: the coefficients of reliability were $.88, .92$, and .99 for psychological aggression, sexual coercion, and physical assault, respectively.

Second, five items pertaining to potentially psychologically abusive situations were adapted from the Appraisals of Ambiguous Situations questionnaire (AASQ; Kelly, Matheson \& Anisman, unpublished). The scale contains a number of situations that could be appraised as threatening or benign. In the present study, the items described situations that could potentially be construed as instances of psychological maltreatment (e.g., "You and your partner are at a bar, and he sees you talking to a male friend. The next day, he does not return your calls"). Participants were asked to appraise each event along four dimensions: threat ("How threatening would this situation be for you?"), distress ("How distressing would this situation be for you?"), control over the situation ("How much control do you think you would have over this event happening in the first place?"), and control over the outcome ("How much control do you think you would have over the outcome of this event?'). As well, participants selected one of five potential outcomes of the event. The threat, distress, and control items were each answered using a Likert scale that ranged from "not at all" (1) to "extremely" (5), while the outcome scale comprised 5 possible outcomes, which ranged from a "positive" outcome (1) to a "negative" outcome (5). A score was obtained on each of the five dimensions (distress, threat, control over 
event, control over outcome, possible outcome) by obtaining the mean across the five questions. Reliability was adequate for threat (.71), distress (.62), control over the event (.74), and control over the outcome (.71). However, the reliability for perceived possible outcome was only .33. As such, this component of the appraisals scale was not used in the analyses.

Stressor challenge. Participants were randomly assigned to a condition in which they were either shown a picture of an abusive situation, a picture of a stressful situation that did not depict abuse (car accident), or a neutral picture (students talking), and asked to speak in front of a video camera for 2 min about what they perceived to be happening in the situation, what the circumstances were leading up to the situation, and the emotional reactions of the individuals in the picture. Following this, they were asked to talk for a minute about what they would do, and what their emotional responses would be, if they were in the situation depicted in the photograph.The abuse and car accident scene pictures were selected from the International Affective Pictures System (numbers 6360 and 9910), whereas the neutral picture of students talking was taken from a university website. Responses were videotaped for the purposes of the stressor challenge only, and were not analyzed.

Cortisol determination. Saliva samples were collected as described in Study 1. A sample was collected at the beginning of every session (following a $15 \mathrm{~min}$ period of relaxation after entering the laboratory), and $15 \mathrm{~min}$ and $30 \mathrm{~min}$ after completion of the speaking task. All samples were maintained at $-80^{\circ} \mathrm{C}$ until subsequent cortisol determinations. Salivary cortisol levels were determined, in duplicate, by means of a solid phase radio-immuno assay using ${ }^{125} \mathrm{I}$ kits obtained from ICN Biomedicals Inc., CA. 
Samples were assayed in a single run to avoid interassay variability. Intrassay variability was less than $10 \%$.

\section{Statistical Analysis}

As in Study 1, regression analyses were conducted to assess the unique effects of each type of abuse (psychological, physical, sexual) on symptoms of depression and PTSD. As well, the correlations between the potential mediating variables were reported. In order to assess the effects of the mediating variables on the relationship between abuse and well-being, multiple mediators were examined simultaneously using the bootstrap method, as described in Study 1. Specifically, in this study, mediational analyses were performed with the all of the coping subscales together as a set, including those of the SCOPE (emotion-focused coping, avoidance, problem-focused coping) as well as those of the ECS (emotional processing and emotional expression). In this way, it would be possible to compare the relative efficacy of each coping dimension with the others. Abuse appraisals (appraisals of psychological, sexual, and physical abuse adapted from the CTSR) were assessed as a third set and finally, the variables pertaining to appraisals of ambiguous events (appraisals of threat, distress, control over the event, and control over the outcome) was a third set of potential mediators.

Changes in cortisol following the challenge were calculated relative to baseline levels. Baseline hormone level and cortisol ratios were analyzed, by a series of hierarchical regression analyses, as a function of stressor condition, abuse, and PTSD symptoms. 


\section{Results}

\section{Correlational Analyses}

Correlations between the predictor and outcome variables. To assess this relation, a multiple regression analysis was conducted in which symptoms were regressed simultaneously onto the three subscales of abuse: psychological aggression, physical assault, and sexual coercion. Abuse significantly predicted both current depressive symptoms $\left(R^{2}=.15, F(3,111=6.73, p<.001)\right.$ and PTSD symptoms $\left(R^{2}=.15, F(3\right.$, $110)=6.28, p<.01$ ) (Table 25.). Sexual coercion and psychological abuse were strongly correlated with depressive symptoms, while physical abuse was not. As well, each of the forms of abuse was positively correlated with PTSD symptoms, although only psychological maltreatment contributed unique variance to symptoms.

Table 25. Regression Analysis (Pearson Correlations and Standardized Regression Coefficients) Assessing the Relations between Abuse and Well-being

\begin{tabular}{|c|c|c|c|c|c|c|}
\hline \multirow{3}{*}{ Abuse } & \multicolumn{3}{|c|}{ Depression } & \multicolumn{3}{|c|}{ PTSD } \\
\hline & $r$ & $\beta$ & $R^{2}$ & $r$ & $\beta$ & $R^{2}$ \\
\hline & & & $.15 * * *$ & & & $.15 * *$ \\
\hline Psychological & $.22 *$ & $.23^{*}$ & & $.37 * * *$ & $.33 * *$ & \\
\hline Physical & -.06 & $-.22 *$ & & $.20 *$ & .04 & \\
\hline Sexual & $.31 * *$ & $.29 * *$ & & $.19^{*}$ & .09 & \\
\hline
\end{tabular}

Correlations among the potential mediating variables. It will be recalled that appraisals and coping were hypothesized to mediate the relationship between abuse and 
well-being (in terms of symptoms of depression and PTSD). Correlational analyses were conducted in order to assess the relationships between the potential mediating variables, coping and appraisals. The correlations are presented in Table 26. Emotion-focused and avoidant coping were related to control over outcome, in that greater use of these strategies was associated with less perceived control over the outcome of the ambiguous events. Avoidant coping was negatively related to perceptions of abuse. That is, greater use of avoidant coping was associated with perceptions of the behaviours on the CTS-R as being less abusive. 
8

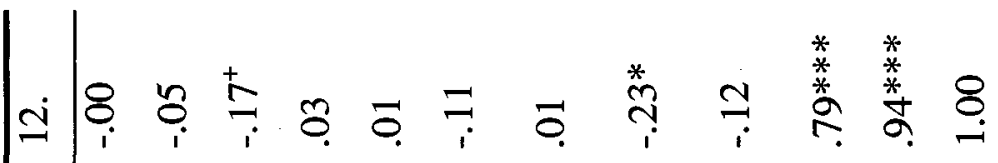

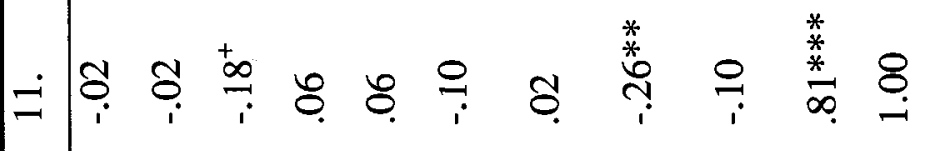

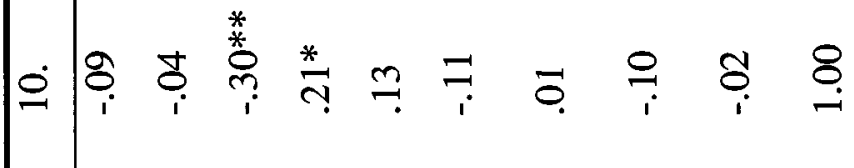

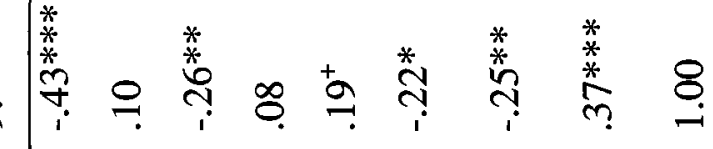

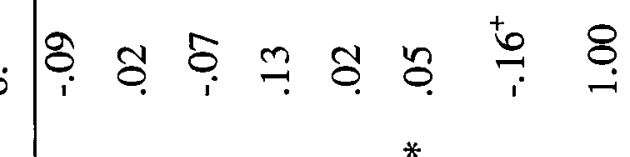

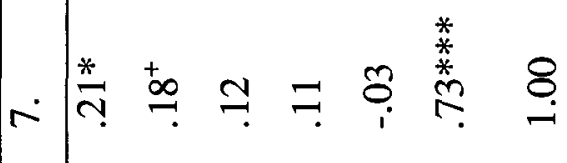

$$
\begin{aligned}
& \text { - }
\end{aligned}
$$

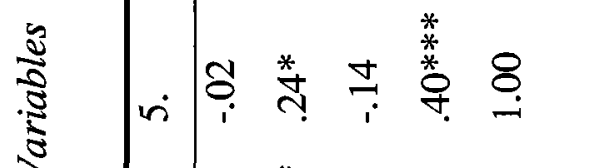

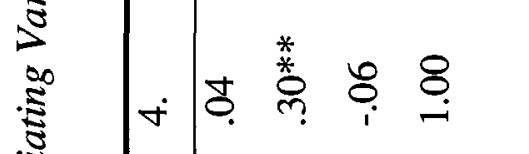

$$
\begin{aligned}
& \text { : }
\end{aligned}
$$

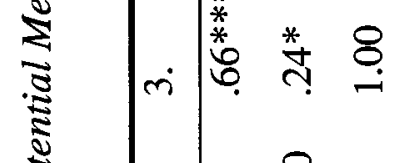

$$
\begin{aligned}
& \left.\frac{2}{0}\right)^{2} \\
& \text { 일 } \\
& \sum_{3}^{3} \\
& \begin{array}{c}
0 \\
0 \\
0 \\
0 \\
0 \\
0 \\
0 \\
0 \\
0 \\
0 \\
0 \\
0 \\
0 \\
0
\end{array} \\
& \text { 告 }
\end{aligned}
$$
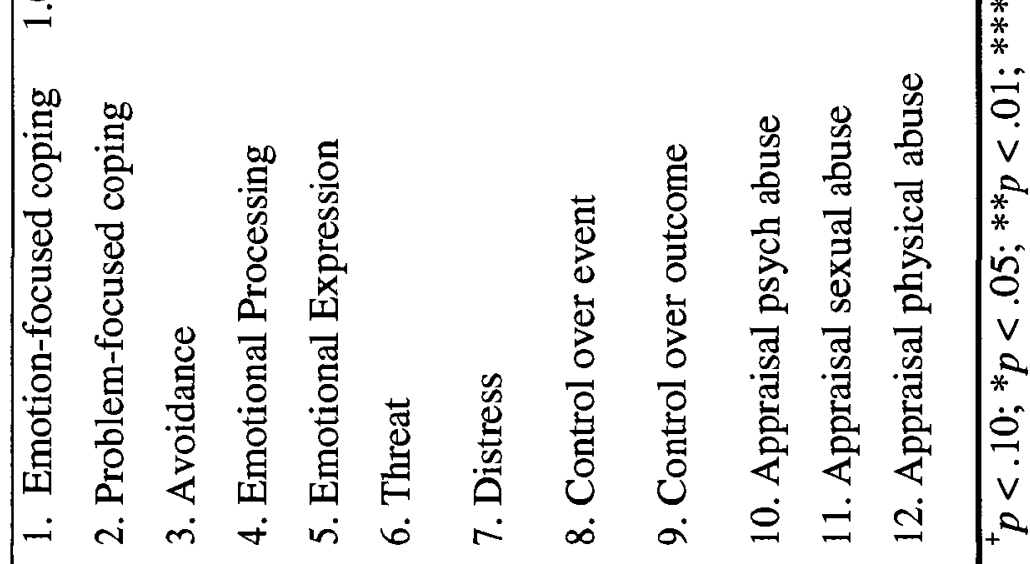


\section{Role of Coping in Mediating the Relationship between Abuse and Well-Being}

First, the potential mediating effects of coping were assessed. As indicated in Figure 2 above, in addition to the direct effects of abuse on symptoms of mental illness, coping styles may mediate the effects of abuse. Specifically, it was hypothesized that abuse would be associated with the increased use of ineffective coping strategies, as found in Study 1, which would lead to increased vulnerability to developing symptoms of depression and PTSD. However, in this study the effects of the more positive emotional approach strategies were also assessed.

Mediation of depression scores by coping. For the present study, since physical assault was unrelated to depressive symptoms, only sexual coercion and psychological aggression were assessed as independent variables in the bootstrap procedure. The coping subscales from the SCOPE (emotion-focused coping, problem-focused coping, and avoidance), as well as those of the ECS (emotional processing and emotional expression), were entered into the analysis together as potential mediators. As indicated in Table 27, psychological abuse was associated with the use of avoidant and emotion-focused coping. Sexual coercion was correlated with emotion-focused coping, and marginally associated with avoidant coping. Finally, physical assault was associated with the use of all of the coping strategies. Unexpectedly, the relationship between physical abuse and problemfocused coping was in the positive direction, although the relationship was only marginally significant. None of the abuse variables were associated with the subscales of the ECS. With respect to the relationship between the mediators and the dependent variable, it was found that depression was associated with the increased use of emotion- 
focused coping strategies, as well as the decreased use of problem-focused coping and emotional expression.

The direct and indirect (i.e., through coping) effects of psychological and sexual abuse on depression are shown in Table 28. Sexual coercion was associated with BDI score, while psychological aggression was marginally related. Once the coping variables were included, the relationship between psychological abuse and depression became nonsignificant, whereas that between sexual abuse and depression was reduced. Thus, overall, the coping variables mediated the effects of psychological abuse on depression, and partially mediated the effects of sexual coercion. The bootstrapping procedure was performed to assess the role of coping as a potential mediator of the effects of relationship abuse on symptoms of depression. As indicated in Table 29, it is evident from the confidence interval that as a set, the coping variables mediate the effect of psychological abuse and sexual coercion on depressive symptoms. However, examination of the specific indirect effects indicated that only emotion-focused coping was a significant mediator for both types of abuse. 
Table 27. Direct Effects of Abuse - Coping and Coping - Depression

\begin{tabular}{|c|c|c|c|c|}
\hline & Coefficient & $S E$ & $t$ & $p$ \\
\hline \multicolumn{5}{|l|}{ IV: Psychological Aggression } \\
\hline \multicolumn{5}{|l|}{ IV-Mediator paths } \\
\hline Psych abuse - Problem-focused & -.00 & .01 & -.23 & .82 \\
\hline Psych abuse - Emotion-focused & .05 & .02 & 2.66 & .01 \\
\hline Psych abuse - Avoidant & .04 & .01 & 2.72 & .01 \\
\hline Psych abuse - Processing & -.01 & .01 & -.80 & .42 \\
\hline Psych abuse - Expression & .00 & .02 & .27 & .79 \\
\hline \multicolumn{5}{|l|}{ Mediator-DV paths } \\
\hline Problem-focused - Depression & -3.12 & 1.01 & -3.09 & .00 \\
\hline Emotion-focused - Depression & 4.52 & .83 & 5.46 & .00 \\
\hline Avoidant - Depression & .53 & 1.17 & .45 & .65 \\
\hline Processing - Depression & -.25 & 1.21 & -.21 & .84 \\
\hline Expression - Depression & -2.53 & .87 & -2.92 & .00 \\
\hline \multicolumn{5}{|l|}{ IV: Sexual Coercion } \\
\hline \multicolumn{5}{|l|}{ IV-Mediator paths } \\
\hline Sexual abuse - Problem-focused & -.10 & .12 & -.87 & .39 \\
\hline Sexual abuse - Emotion-focused & .42 & .17 & 2.47 & .02 \\
\hline Sexual abuse - Avoidant & .21 & .13 & 1.70 & .09 \\
\hline Sexual abuse - Processing & -.02 & .10 & -.15 & .89 \\
\hline Sexual abuse - Expression & -.05 & .14 & -.34 & .73 \\
\hline \multicolumn{5}{|l|}{ Mediator - DV paths } \\
\hline Problem-focused - Depression & -2.90 & .99 & -2.92 & .00 \\
\hline Emotion-focused - Depression & 4.26 & .82 & 5.22 & .00 \\
\hline Avoidant - Depression & .52 & 1.13 & .46 & .65 \\
\hline Processing - Depression & -.35 & 1.18 & -.30 & .77 \\
\hline Expression - Depression & -2.49 & .84 & -2.95 & .00 \\
\hline \multicolumn{5}{|l|}{$\begin{array}{l}\text { IV: Physical Assault } \\
\text { IV-Mediator paths }\end{array}$} \\
\hline Physical abuse - Problem-focused & .25 & .13 & 1.96 & .05 \\
\hline Physical abuse - Emotion-focused & .48 & .18 & 2.66 & .01 \\
\hline Physical abuse - Avoidant & .34 & .13 & 2.55 & .01 \\
\hline Physical abuse - Processing & .06 & .11 & .53 & .60 \\
\hline Physical abuse - Expression & .23 & .16 & 1.46 & .15 \\
\hline
\end{tabular}


Table 26 cont'd

\begin{tabular}{lrrrr}
\hline Mediator-DV paths & -2.91 & 1.00 & -2.92 & .00 \\
Problem-focused - Depression & 4.76 & .81 & 5.86 & .00 \\
Emotion-focused - Depression & .86 & 1.14 & .76 & .45 \\
Avoidant - Depression & -.40 & 1.18 & -.34 & .74 \\
Processing - Depression & -2.24 & .85 & -2.63 & .01 \\
Expression - Depression & & & \\
\hline
\end{tabular}

Table 28. Direct and Indirect (through Coping) Effects of Abuse on Symptoms of

Depression: Results of Bootstrap Analysis

\begin{tabular}{lrrrr}
\hline Relationship with BDI Score & Coefficient & \multicolumn{1}{l}{$S E$} & \multicolumn{1}{l}{$t$} & $p$ \\
\hline Direct Effects & & & & \\
$\quad$ Psychological abuse & .31 & .17 & 1.88 & .06 \\
$\quad$ Sexual coercion & 4.68 & 1.41 & 3.33 & .00 \\
& & & & \\
Indirect Effects (through & & & & \\
mediator) & & & & \\
$\quad$ Psychological abuse & .06 & .13 & .45 & .65 \\
$\quad$ Sexual coercion & 2.35 & 1.13 & 2.09 & .04 \\
\hline
\end{tabular}


Table 29. Bootstrap Confidence Intervals for the Total and Specific Mediating Effects of Coping on the Psychological Abuse-Depression Relationship

\begin{tabular}{|c|c|c|}
\hline & \multicolumn{2}{|c|}{ Bootstrapping 95\% CI } \\
\hline & Lower & Upper \\
\hline \multicolumn{3}{|l|}{ Psychological Aggression } \\
\hline Problem-focused coping & -.08 & .11 \\
\hline Emotion-focused coping & .06 & .46 \\
\hline Avoidant coping & -.05 & .14 \\
\hline Emotional processing & -.03 & .05 \\
\hline Emotional expression & -.11 & .07 \\
\hline TOTAL & .03 & .49 \\
\hline \multicolumn{3}{|l|}{ Sexual Coercion } \\
\hline Problem-focused coping & -.24 & 1.48 \\
\hline Emotion-focused coping & .54 & 3.73 \\
\hline Avoidant coping & -.28 & .98 \\
\hline Emotional processing & -.19 & .33 \\
\hline Emotional expression & -.50 & .99 \\
\hline TOTAL & .56 & 4.55 \\
\hline
\end{tabular}

Mediation of PTSD scores by coping. As in the previous analysis, psychological aggression was associated with the increased use of emotion-focused and avoidant coping strategies. Sexual coercion was associated with emotion-focused coping, and was marginally related to avoidant coping. Finally, physical assault was correlated with emotion-focused and avoidant coping, but only marginally related to problem-focused coping (Table 30). PTSD was associated with the increased use of emotion-focused and avoidant coping strategies, and the decreased use of problem-focused coping. 
As with depression, all of the coping dimensions, both from the SCOPE and the ECS, were entered into the analysis together as potential mediators. As shown in Table 31, although the total effect of each type of abuse on PTSD was significant, the associations between sexual and physical abuse and PTSD became non-significant when the coping dimensions were included as mediators, while that between psychological aggression and PTSD became only marginally significant. Thus, coping was at least a partial mediator in the relationship between abuse and PTSD. As indicated in Table 32, overall, the coping dimensions mediated the effects of all of the types of abuse on symptoms of PTSD. Of the specific indirect effects, it was found that both emotionfocused and avoidant coping were mediators in the relationship between psychological aggression and PTSD. However, as the pairwise contrast indicated, emotion-focused coping was not different from avoidant coping in its effectiveness as a mediator. Emotion-focused coping was the only significant mediator in the relationship between sexual coercion and PTSD. Finally, emotion-focused, avoidant, and problem-focused coping were all significant mediators in the relationship between physical assault and PTSD. However, it is evident from the pairwise contrasts that emotion-focused and avoidant coping were equally effective as mediators in this relationship. 
Table 30. Direct Effects of Abuse - Coping and Coping - PTSD

\begin{tabular}{|c|c|c|c|c|}
\hline & Coefficient & $S E$ & $t$ & $p$ \\
\hline \multicolumn{5}{|l|}{ IV: Psychological Aggression } \\
\hline \multicolumn{5}{|l|}{ IV-Mediator paths } \\
\hline Psych abuse - Problem-focused & -.01 & .01 & -.46 & .65 \\
\hline Psych abuse - Emotion-focused & .06 & .02 & 2.98 & .00 \\
\hline Psych abuse - Avoidant & .04 & .01 & 2.61 & .01 \\
\hline Psych abuse - Processing & -.01 & .01 & -.58 & .57 \\
\hline Psych abuse - Expression & .01 & .02 & .40 & .69 \\
\hline \multicolumn{5}{|l|}{ Mediator-DV paths } \\
\hline Problem-focused - PTSD & -7.36 & 1.95 & -3.77 & .00 \\
\hline Emotion-focused - PTSD & 10.17 & 1.58 & 6.44 & .00 \\
\hline Avoidant - PTSD & 7.20 & 2.26 & 3.21 & .00 \\
\hline Processing - PTSD & 5.79 & 2.34 & 2.47 & .02 \\
\hline Expression - PTSD & .01 & 1.69 & -.11 & .91 \\
\hline \multicolumn{5}{|l|}{ IV: Sexual Coercion } \\
\hline \multicolumn{5}{|l|}{ IV-Mediator paths } \\
\hline Sexual abuse - Problem-focused & -.12 & .12 & -1.01 & .32 \\
\hline Sexual abuse - Emotion-focused & .45 & .17 & 2.68 & .01 \\
\hline Sexual abuse - Avoidant & .21 & .12 & 1.65 & .10 \\
\hline Sexual abuse - Processing & -.00 & .10 & -.01 & .99 \\
\hline Sexual abuse - Expression & -.04 & .14 & -.26 & .80 \\
\hline \multicolumn{5}{|l|}{ Mediator-DV paths } \\
\hline Problem-focused - PTSD & -7.75 & 2.00 & -3.88 & .00 \\
\hline Emotion-focused - PTSD & 10.72 & 1.62 & 6.62 & .00 \\
\hline Avoidant - PTSD & 7.74 & 2.27 & 3.40 & .00 \\
\hline Processing - PTSD & 5.47 & 2.38 & 2.30 & .02 \\
\hline Expression - PTSD & .17 & 1.71 & .10 & .92 \\
\hline \multicolumn{5}{|l|}{ IV: Physical Assault } \\
\hline \multicolumn{5}{|l|}{ IV-Mediator paths } \\
\hline Physical abuse - Problem-focused & .22 & .13 & 1.74 & .09 \\
\hline Physical abuse - Emotion-focused & .53 & .18 & 2.91 & .00 \\
\hline Physical abuse - Avoidant & .33 & .13 & 2.48 & .01 \\
\hline Physical abuse - Processing & .08 & .11 & .68 & .49 \\
\hline Physical abuse - Expression & .24 & .15 & 1.55 & .12 \\
\hline
\end{tabular}


Table 29 cont'd

\begin{tabular}{lrrrr}
\hline Mediator-DV paths & & & & \\
Problem-focused - PTSD & -7.76 & 1.99 & -3.90 & .00 \\
Emotion-focused - PTSD & 10.60 & 1.61 & 6.59 & .00 \\
Avoidant - PTSD & 7.67 & 2.28 & 3.36 & .00 \\
Processing - PTSD & 5.48 & 2.38 & 2.31 & .02 \\
Expression - PTSD & .10 & 1.73 & .06 & .95 \\
\hline
\end{tabular}

Table 31. Direct and Indirect (through Coping) Effects of Abuse on Symptoms of PTSD:

Results of Bootstrap Analysis

\begin{tabular}{lrrrr}
\hline Relationship with IES Score & Coefficient & \multicolumn{1}{l}{ SE } & \multicolumn{2}{l}{$p$} \\
\hline Direct Effects & & & & \\
$\quad$ Psychological abuse & 1.34 & .36 & 3.72 & .00 \\
$\quad$ Sexual coercion & 6.49 & 3.19 & 2.04 & .04 \\
$\quad$ Physical assault & 7.50 & 3.58 & 2.09 & .04 \\
& & & & \\
Indirect Effects (through & & & & \\
mediator) & & & & \\
$\quad$ Psychological abuse & .50 & .26 & 1.93 & .06 \\
$\quad$ Sexual coercion & -.96 & 2.27 & -.42 & .67 \\
$\quad$ Physical assault & .64 & 2.52 & .25 & .80 \\
\hline
\end{tabular}


Table 32. Bootstrap Confidence Intervals and Contrasts for the Total and Specific

Mediating Effects of Coping on the Psychological Abuse-PTSD Relationship

\begin{tabular}{|c|c|c|}
\hline & \multicolumn{2}{|c|}{ Bootstrapping 95\% CI } \\
\hline & Lower & Upper \\
\hline \multicolumn{3}{|l|}{ Psychological Aggression } \\
\hline Problem-focused coping & -.15 & .26 \\
\hline Emotion-focused coping & .21 & 1.06 \\
\hline Avoidant coping & .05 & .61 \\
\hline Emotional processing & -.21 & .08 \\
\hline Emotional expression & -.07 & .07 \\
\hline TOTAL & .30 & 1.38 \\
\hline \multicolumn{3}{|l|}{ Contrasts } \\
\hline Emotion-focused vs. Avoidant & -.15 & .86 \\
\hline \multicolumn{3}{|l|}{ Sexual Coercion } \\
\hline Problem-focused coping & -.73 & 3.16 \\
\hline Emotion-focused coping & 1.53 & 9.21 \\
\hline Avoidant coping & -.06 & 4.69 \\
\hline Emotional processing & -1.45 & 1.16 \\
\hline Emotional expression & -.73 & .45 \\
\hline TOTAL & 2.45 & 12.81 \\
\hline \multicolumn{3}{|l|}{ Physical Assault } \\
\hline Problem-focused coping & -4.71 & -.05 \\
\hline Emotion-focused coping & 1.94 & 10.56 \\
\hline Avoidant coping & .33 & 6.04 \\
\hline Emotional processing & -.44 & 2.02 \\
\hline Emotional expression & -.80 & 1.37 \\
\hline TOTAL & .90 & 12.72 \\
\hline \multicolumn{3}{|l|}{ Contrasts } \\
\hline Emotion-focused vs. Avoidant & -1.45 & 8.69 \\
\hline Problem-focused vs. Emotion-focused & -1.07 & -.08 \\
\hline Problem-focused vs. Avoidant & -.67 & .10 \\
\hline
\end{tabular}


Role of Appraisals in Mediating the Relationship between Abuse and Well-Being

The mediating role of women's appraisals of behaviours within a relationship was assessed. As indicated in Figure 2, abuse may be associated with altered appraisals of events within a relationship, which could lead to symptoms of depression and PTSD. Mediation of depression scores by abuse appraisals. As indicated in Table 33, sexual coercion was associated with the perception that the behaviours from the CTS-R were less abusive. As well, physical assault was marginally inversely related to perceptions of psychological abuse. However, appraisals of abuse were unrelated to depressive symptoms. It was clear that appraisals of abusive behaviours did not mediate the effects of abuse on depression, since the mediator-dependent variable relationship must be significant in order for a mediation effect to be present (Collins et al., 1998). A bootstrap analysis was not performed on the data. 
Table 33. Direct Effects of Abuse - Appraisals and Appraisals - Depression

\section{Coefficient}

IV: Psychological Aggression

IV-Mediator paths

Psych abuse - Psych abuse appraisal

Psych abuse - Sexual abuse appraisal

Psych abuse - Physical abuse appraisal

Mediator-DV paths

Psych abuse appraisal - Depression

Sexual abuse appraisal - Depression

Physical abuse appraisal - Depression

IV: Sexual Coercion

IV-Mediator paths

Sexual abuse - Psych abuse appraisal

Sexual abuse - Sexual abuse appraisal

Sexual abuse - Physical abuse

Mediator-DV paths

Psych abuse appraisal - Depression

Sexual abuse appraisal - Depression

Physical abuse appraisal - Depression

IV: Physical Assault

IV-Mediator paths

Physical abuse - Psych abuse appraisal

Physical abuse - Sexual abuse

Physical abuse - Physical abuse

Mediator-DV paths

Psych abuse appraisal - Depression

Sexual abuse appraisal - Depression

Physical abuse appraisal - Depression
SE

$-.22$

$-.03$

$-.03$

$-.12$

.10

.04

$-2.42$

$-4.15$

$-5.61$

$-.27$

.40

$-.01$

$-2.31$

$-.53$

.14

1.27

1.43

2.41

.20

$-1.36$

.18

.31

1.32

.19

.18

$-.03$

.98
.11

.85

.89

.57

.76

.85

$\begin{array}{ll}.31 & .76 \\ .85 & .85\end{array}$


Mediation of depression scores by appraisals of ambiguous events. As shown in Table 34, extent of psychological aggression was associated with perceptions of lack of control over the outcome of potentially ambiguous events. As well, physical assault was associated with heightened perceptions of threat and distress associated with such events. Control over the outcome was significantly related to depressive symptoms.

As indicated in Table 35, the relationship between psychological abuse and depression became non-significant when appraisals were included as mediators in the model. In addition, the effects of sexual coercion on depression were reduced when appraisals were considered. Thus, the association between psychological abuse and depression was mediated by appraisals, while that between sexual abuse and depression was partially mediated. The bootstrapping procedure was performed to determine whether the specific dimensions of appraisals of ambiguous events (i.e., perceptions of threat, distress, control over event, and control over outcome, as they pertain to events within a relationship) mediated the effects of abuse on depressive symptoms. The four appraisals subscales were entered in the model as potential mediators. As indicated in Table 36, perceived control over the outcome of ambiguous events was a mediator in the relationship between psychological abuse and depression, since the confidence interval of the indirect effect did not contain zero. The direction of the direct effects (Table 34) suggests that experiences of psychological aggression are associated with a decrease in perceived control over the outcome of events within a relationship, which is then associated with greater symptoms of depression. 
Table 34. Direct Effects of Abuse - Appraisals of Ambiguous Events and Appraisals -

Depression

Coefficient

IV: Psychological Aggression

IV-Mediator paths

Psych abuse - Threat

Psych abuse - Distress

Psych abuse - Control over event

Psych abuse - Control over outcome

Mediator-DV paths

Threat - Depression

Distress - Depression

Control over event - Depression

Control over outcome - Depression

IV: Sexual Coercion

IV-Mediator paths

Sexual abuse - Threat

Sexual abuse - Distress

Sexual abuse - Control over event

Sexual abuse - Control over outcome

Mediator-DV paths

Threat - Depression

Distress - Depression

Control over event - Depression

Control over outcome - Depression

\section{Control over outcome - Depression}

$-.89$

1.51

$S E$

.02

.00

$-.02$

$-.03$

$-1.15$

1.98

.30

$-3.49$

.13

.13

.02

$-.12$

.06

$-3.47$
.02

.02

.02

.01

\subsection{8}

1.42

.88

1.16

$-.90$

1.39

.34

$-3.00$

.95

.14

$-1.08$

$-2.08$

$p$

IV: Physical Assault

IV-Mediator paths

Physical abuse - Threat

Physical abuse - Control over event

$-.12$

Physical abuse - Control over outcome

.17

.15

.17

.13

1.22

1.36

.85

1.11
.77

.84

.10

$-.91$

$-.73$

1.11

.07

$-3.13$
.35

.89

.28

.04

.37

.17

.74

.00

Mediator-DV paths

Threat - Depression

$-.79$

1.79

1.28

$-.62$

1.77

.18

1.78

.08

.16

$-.65$

.08

.19

.52

Distress - Depression

.18

1.42

1.27

Control over event - Depression

$-3.93$

.88

.20

1.16

$-3.39$

Control over outcome - Depression

1.16

.00 
Table 35. Direct and Indirect (through Appraisals) Effects of Abuse on Symptoms of Depression: Results of Bootstrap Analysis

\begin{tabular}{lrrrr}
\hline Relationship with BDI Score & Coefficient & \multicolumn{1}{l}{$S E$} & \multicolumn{1}{l}{$t$} & $p$ \\
\hline Direct Effects & & & & \\
$\quad$ Psychological abuse & .34 & .16 & 2.05 & .04 \\
$\quad$ Sexual coercion & 4.81 & 1.39 & 3.47 & .00 \\
& & & & \\
Indirect Effects (through & & & & \\
mediator) & & & & \\
$\quad$ Psychological abuse & .25 & .16 & 1.56 & .12 \\
$\quad$ Sexual coercion & 4.32 & 1.33 & 3.25 & .00 \\
\hline
\end{tabular}


Table 36. Bootstrap Confidence Intervals for the Total and Specific Indirect Effects of Appraisals of Ambiguous Events on the Psychological Abuse-Depression Relationship

\begin{tabular}{lcc}
\hline & \multicolumn{2}{c}{ Bootstrapping 95\% CI } \\
\hline & Lower & Upper \\
Psychological Aggression & & \\
Threat & -.15 & .02 \\
Distress & -.05 & .10 \\
Control over Event & -.07 & .03 \\
Control over Outcome & .02 & .27 \\
TOTAL & -.03 & .26 \\
& & \\
Sexual Coercion & & \\
Threat & -1.09 & .12 \\
Distress & -.13 & 1.21 \\
Control over Event & -.25 & .31 \\
Control over Outcome & -.38 & 1.75 \\
TOTAL & -.46 & 1.95 \\
\hline
\end{tabular}

Mediation of PTSD scores by abuse appraisals. As in the previous analysis, the direct relation of sexual coercion to appraisals of abusive behaviours was significant, such that experiences of sexual abuse were associated with decreased perceptions that behaviours were abusive (Table 37). However, the correlations between appraisals and symptoms of PTSD were not significant. Therefore, the mediated relationship was not significant. 
Table 37. Direct Effects of Abuse - Appraisals and Appraisals - PTSD

\begin{tabular}{lrrrr}
\hline & Coefficient & \multicolumn{1}{l}{$S E$} & \multicolumn{1}{l}{$t$} & \multicolumn{1}{l}{$p$} \\
\hline IV: Psychological Aggression & & & & \\
IV-Mediator paths & -.16 & .14 & -1.19 & .24 \\
$\quad$ Psych abuse - Psych abuse appraisal &. .01 & .15 & -.10 & .92 \\
Psych abuse - Sexual abuse appraisal & .01 & .25 & .05 & .96 \\
Psych abuse - Physical abuse appraisal & & & & \\
Mediator-DV paths & & & & \\
$\quad$ Psych abuse appraisal - PTSD & -.12 & .47 & -.25 & .80 \\
Sexual abuse appraisal - PTSD & -.23 & .72 & -.31 & .75 \\
Physical abuse appraisal - PTSD & .25 & .44 & .57 & .57 \\
& & & & \\
IV: Sexual Coercion & & & & \\
IV-Mediator paths & & & & \\
Sexual abuse - Psych abuse appraisal & -2.13 & 1.19 & -1.78 & .08 \\
Sexual abuse - Sexual abuse appraisal & -4.06 & 1.27 & -3.21 & .00 \\
Sexual abuse - Physical abuse appraisal & -5.31 & 2.17 & -2.44 & .02 \\
& & & & \\
Mediator-DV paths & & & & \\
Psych abuse appraisal - PTSD & -.55 & .48 & -1.15 & .25 \\
Sexual abuse appraisal - PTSD & .24 & .77 & .31 & .75 \\
Physical abuse appraisal - PTSD & .27 & .45 & .59 & .55 \\
IV: Physical Assault & & & & \\
IV-Mediator paths & & & & \\
Physical abuse - Psych abuse appraisal & -1.92 & 1.29 & -1.48 & .14 \\
Physical abuse - Sexual abuse appraisal & -.42 & 1.43 & -.29 & .77 \\
Physical abuse - Physical abuse appraisal & .44 & 2.41 & .18 & .85 \\
Mediator-DV paths & & & & \\
Psych abuse appraisal - PTSD & -.22 & .50 & -.45 & .65 \\
Sexual abuse appraisal - PTSD &. .11 & .75 & -.14 & .89 \\
Physical abuse appraisal - PTSD & .47 & .49 & .62 \\
\hline
\end{tabular}

Mediation of PTSD scores by appraisals of ambiguous events. Finally, the potential mediating effects of appraisals of ambiguous events was examined. As indicated earlier, psychological maltreatment was associated with appraisals of less control over the 
outcome of events. As well, perceived control over the outcome was negatively correlated with PTSD (Table 38).

As shown in Table 39, the effects of psychological and sexual abuse on PTSD were reduced when appraisals were considered as mediators, whereas the effects of physical abuse became non-significant. A bootstrap analysis was then performed to assess the potential mediating effects of the specific dimensions of appraisals on PTSD symptoms. As indicated in Table 40, perceived control over the outcome of ambiguous events mediated the relationship between psychological aggression and PTSD, since the confidence interval of the indirect effect did not contain zero. 
Table 38. Direct Effects of Abuse - Appraisals of Ambiguous Event and Appraisals -

PTSD

$\begin{array}{llll}\text { Coefficient } & S E & t & p\end{array}$

IV: Psychological Aggression

IV-Mediator paths

Psych abuse - Threat

$\begin{array}{llll}.01 & .02 & .45 & .65\end{array}$

Psych abuse - Distress

$.00 \quad .02$

.07

.95

Psych abuse - Control over event

$-.02$

.02

$-1.15$

.25

Psych abuse - Control over outcome

$-.03$

.01

$-2.16$

.03

Mediator-DV paths

Threat - PTSD

1.84

2.82

.65

.51

Distress - PTSD

$-1.94$

3.10

$-.63$

.53

Control over event - PTSD

2.99

1.94

1.54

.13

Control over outcome - PTSD

$-10.39$

2.55

$-4.08$

.00

IV: Sexual Coercion

IV-Mediator paths

Sexual abuse - Threat

.07

Sexual abuse - Distress

.12

Sexual abuse - Control over event

Sexual abuse - Control over outcome

$-.13$

17

.79

.43

.98

$.17-.02$

.33

Mediator-DV paths

Threat - PTSD

2.62

.13

$-.98$

Distress - PTSD

$-3.15$

2.90

.91

.37

2.42

3.19

$-.99$

.33

Control over event - PTSD

1.99

1.22

.23

Control over outcome - PTSD

$-11.28$

2.59

$-4.35$

.00

IV: Physical Assault

IV-Mediator paths

Physical abuse - Threat

Physical abuse - Distress

.25

.27

Physical abuse - Control over event

$-.13$

$-.24$

.18

1.39

.17

.16

1.70

.09

Physical abuse - Control over outcome

.19

$-.68$

.50

$.14 \quad-1.69$

.09

Mediator-DV paths

Threat - PTSD

2.33

$-3.11$

2.91

.80

.42

Distress - PTSD

2.64

3.20

$-.97$

.33

Control over event - PTSD

2.00

1.32

.19

Control over outcome - PTSD

$-11.26$

2.61

$-4.31$

.00


Table 39. Direct and Indirect (through Appraisals) Effects of Abuse on Symptoms of PTSD: Results of Bootstrap Analysis

\begin{tabular}{lrrrr}
\hline Relationship with IES Score & Coefficient & \multicolumn{1}{l}{ SE } & \multicolumn{1}{l}{$p$} \\
\hline Direct Effects & & & & \\
$\quad$ Psychological abuse & 1.34 & .36 & 3.72 & .00 \\
$\quad$ Sexual coercion & 7.05 & 3.32 & 2.12 & .04 \\
$\quad$ Physical assault & 7.50 & 3.58 & 2.09 & .04 \\
& & & & \\
Indirect Effects (through & & & & .00 \\
mediator) & & & & .06 \\
$\quad$ Psychological abuse & 1.07 & .35 & 3.09 & .11 \\
$\quad$ Sexual coercion & 5.78 & 3.08 & 1.88 & \\
$\quad$ Physical assault & 5.44 & 3.39 & 1.60 & \\
\hline
\end{tabular}


Table 40. Bootstrap Confidence Intervals for the Total and Specific Indirect Effects of Appraisals of Ambiguous Events on the Psychological Abuse-PTSD Relationship

\begin{tabular}{lrr}
\hline & \multicolumn{2}{c}{ Bootstrapping 95\% CI } \\
\hline \multicolumn{2}{c}{ Lower } \\
Psychological Aggression & \multicolumn{2}{c}{ Upper } \\
Threat & -.06 & .25 \\
Distress & -.17 & .08 \\
Control over Event & -.33 & .03 \\
Control over Outcome & .08 & .69 \\
TOTAL & -.05 & .66 \\
& & \\
Sexual Coercion & & \\
Threat & -.59 & 2.30 \\
Distress & -3.21 & .37 \\
Control over Event & -1.15 & 1.18 \\
Control over Outcome & -1.36 & 4.80 \\
TOTAL & -1.75 & 4.70 \\
& & \\
Physical Assault & & \\
Threat & -.41 & 3.45 \\
Distress & -4.47 & .37 \\
Control over Event & -2.49 & .40 \\
Control over Outcome & -.31 & 6.90 \\
TOTAL & -1.41 & 6.08 \\
\hline
\end{tabular}

Role of Appraisals in Mediating the Relationship between Abuse and Coping

As indicated in the preceding sections, coping and appraisals can operate in a reciprocal fashion, such that appraisals can influence the coping styles selected, and coping can in turn lead to reinterpretation of events (Chang \& Strunk, 1999; Folkman \& Lazarus, 1985). Thus, appraisals were examined as potential mediators in the relationship between experiences of abuse and coping styles selected. Appraisals may influence symptoms of depression and PTSD indirectly through their effects on coping. As indicated in Table 34 (above), which shows the direct effects of abuse on appraisals, it is evident that psychological abuse was associated with perceptions of low control over the 
outcome of events. As well, control over outcome was in turn associated with both emotion-focused and avoidant coping. Thus, the effect of appraisals of control in the relationship between psychological aggression and ineffective coping styles was the only possible mediating model. This was assessed using the bootstrap procedure as described earlier.

The direct and indirect effects of abuse on coping are shown in Table 41. The relationship between psychological abuse and coping was reduced when appraisals of control was considered as a mediator, although it was still significant. As well, as seen in Table 42, it is evident that the bootstrapped confidence interval for this relationship did not contain zero. Thus, appraisals of control partially mediated the effects of psychological maltreatment on emotion-focused coping. However, this effect was very small.

Table 41. Direct and Indirect (through Appraisals) Effects of Psychological Abuse on Coping: Results of Bootstrap Analysis

\begin{tabular}{lllll}
\hline & Coefficient & $S E$ & $t$ & $p$ \\
\hline $\begin{array}{l}\text { Effect of psychological abuse on } \\
\text { emotion-focused coping }\end{array}$ & & & & \\
$\quad$ Direct & .06 & .02 & 2.98 & .00 \\
$\quad$ Indirect (through appraisals of & .04 & .02 & 2.26 & .03 \\
$\quad$ control over outcome) & & & & \\
& & & & \\
$\begin{array}{l}\text { Effect of psychological abuse on } \\
\text { avoidant coping }\end{array}$ & & & & \\
$\quad \begin{array}{l}\text { Direct } \\
\text { Indirect (through appraisals of } \\
\text { control over outcome) }\end{array}$ & .04 & .01 & 2.61 & .01 \\
\hline
\end{tabular}


Table 42. Bootstrap Confidence Intervals for the Total and Specific Indirect Effects of Appraisals on the Abuse-Coping Relationship

\begin{tabular}{lcc}
\hline & \multicolumn{2}{c}{ Bootstrapping 95\% CI } \\
\hline & Lower & Upper \\
$\begin{array}{l}\text { Psychological aggression \& } \\
\text { emotion-focused coping: }\end{array}$ & .00 & .04 \\
Control over outcome as mediator & & \\
& & \\
$\begin{array}{l}\text { Psychological aggression \& } \\
\text { avoidant coping: } \\
\text { Control over outcome as mediator }\end{array}$ & .00 & .02 \\
\hline
\end{tabular}

Summary of mediating relationships. In summary, consistent with Study 1, emotion-focused coping emerged most consistently as a mediator in the relationship between abuse and symptoms of psychological distress. In addition, avoidant coping was a mediator in the relationship of both psychological and physical abuse to PTSD.

However, avoidant and emotion-focused coping were equally effective mediators in this relationship. Appraisals of control over the outcome of ambiguous events mediated the association between psychological aggression and symptoms of depression and PTSD. Furthermore, appraisals of control mediated the effects of psychological aggression on use of emotion-focused coping, although this effect was small. When alternative mediation models were tested, using mental health symptoms as the independent variable and abuse as the dependent variable, these were not found to be significant except for a marginal effect for emotion-focused coping as a mediator in the relationship between depression and abuse (data not shown), indicating that coping and appraisals were mediators of the impact of abuse on psychological distress, and not vice versa. 


\section{Neuroendocrine Response Elicited by a Challenge}

As in Study 1, the analyses involving cortisol excluded those individuals taking anti-inflammatory and antidepressant medications. However, there were no differences found based on whether women were taking these medications, so a further set of analyses, including these participants, was undertaken. Only the latter analyses are reported here. Furthermore, consistent with Study 1, use of oral contraceptives was statistically controlled for in the analyses in order to partial out its potential effects on cortisol. Mean levels of cortisol at baseline and in response to the challenge are shown in Table 43.

Table 43. Cortisol Levels ( $\mu \mathrm{g} / \mathrm{dl})$ as a Function of Abuse Type

\begin{tabular}{lcccccc}
\hline & Baseline & \multicolumn{2}{c}{$\begin{array}{c}\text { 15 min after } \\
\text { challenge }\end{array}$} & \multicolumn{2}{c}{$\begin{array}{c}\text { 30 min after } \\
\text { challenge }\end{array}$} \\
\hline Mean & SE & Mean & SE & Mean & SE \\
\hline Abuse type & & & & & & \\
$\quad$ Psychological & 1.15 & .08 & 1.05 & .08 & .93 & .06 \\
Physical & 1.11 & .14 & .97 & .13 & .87 & .10 \\
Sexual & 1.09 & .10 & .88 & .10 & .91 & .07 \\
& & & & & & \\
No abuse & 1.10 & .10 & 1.18 & .10 & 1.00 & .08 \\
\hline
\end{tabular}

A regression analysis conducted in which the three types of abuse were regressed against baseline cortisol indicated that abused and non-abused women could not be distinguished on the basis of baseline cortisol levels (Table 44). 
Table 44. Relationship of Abuse to Baseline Cortisol Levels

\begin{tabular}{lrrr}
\hline & \multicolumn{1}{c}{$r$} & \multicolumn{1}{c}{$R^{2}$} \\
\cline { 2 - 4 } Abuse type & & & .003 \\
Psychological & .04 & .04 & \\
Physical & -.00 & -.03 & \\
Sexual & .03 & .02 & \\
\hline
\end{tabular}

Next, a hierarchical polynomial regression analysis was conducted on cortisol ratios following the challenge. Two dummy variables were created to represent the three stressor conditions (abuse picture, accident picture, neutral picture). Specifically, for the first dummy variable, individuals who received the abuse picture were coded with a value of 1 , while those in the other conditions were coded with 0 . For the second dummy variable, those individuals with the accident scene were coded with 1 and the others were coded as 0 . The abuse variables (psychological, sexual or physical) were entered on the first step of the regression, followed by the two dummy variables, IES, IES (quadratic), the set of interactions of the dummy variables with abuse and PTSD (condition*abuse, condition*IES, condition*IES [quadratic]), and finally, the set of interactions of abuse by PTSD (abuse*IES, and abuse*IES [quadratic]) ${ }^{7}$. As indicated in Tables 45-50, neither abuse nor stressor condition moderated cortisol reactions to the challenge.

\footnotetext{
${ }^{7}$ As in Study 1, to compute the interaction terms in the analyses, standardized ( $z$ ) scores were obtained for psychological abuse and PTSD by subtracting each variable's mean from the individual cases and then dividing by the standard deviation. The cross-products were then computed using these standardized scores in order to examine the interactive effects of abuse, condition, and PTSD.
} 
Table 45. Regression Coefficients predicting 15 min Cortisol Ratio as a Function of Psychological Abuse, PTSD Symptoms and Stressor Challenge

\begin{tabular}{|c|c|c|c|}
\hline & $r$ & $B^{8}$ & $R_{\text {change }}^{2}$ \\
\hline \multicolumn{4}{|l|}{ Ratio of $15 \mathrm{~min}$ post-challenge/baseline } \\
\hline Psychological abuse & -.03 & -.03 & .00 \\
\hline Condition & & & .01 \\
\hline Dummy variable 1 & .11 & .12 & \\
\hline Dummy variable 2 & -.03 & .03 & \\
\hline PTSD symptoms & -.06 & -.06 & .00 \\
\hline PTSD symptoms (quadratic) & -.10 & -.52 & .02 \\
\hline $\begin{array}{l}\text { Interactions of Condition and Abuse, and Condition } \\
\text { and PTSD } \\
\text { Condition (dummy 1) * PTSD }\end{array}$ & -.12 & .33 & .04 \\
\hline Condition (dummy 2 ) $*$ PTSD & .15 & -.08 & \\
\hline Condition (dummy 1) * PTSD (quadratic) & -.05 & -.45 & \\
\hline Condition (dummy 2) * PTSD (quadratic) & .11 & .32 & \\
\hline Psych Abuse * Condition (dummy 1) & -.13 & -.05 & \\
\hline Psych Abuse * Condition (dummy 2) & .11 & .05 & \\
\hline Interactions of Abuse and PTSD & & & .00 \\
\hline Psych Abuse * PTSD & -.01 & .08 & \\
\hline Psych Abuse * PTSD (quadratic) & -.04 & .00 & \\
\hline
\end{tabular}

\footnotetext{
${ }^{8}$ While the proportion of variance explained is the contribution made on each step of the hierarchical regression, the regression coefficients represent final step weights.
} 
Table 46. Regression Coefficients predicting 30 min Cortisol Ratio as a Function of Psychological Abuse, PTSD Symptoms and Stressor Challenge

\begin{tabular}{llll}
\hline & $r$ & $B$ & $R_{\text {change }}^{2}$ \\
\hline Ratio of 30 min post-challenge/baseline & & & \\
Psychological abuse & -.11 & -.11 & .01 \\
Condition & & & .00 \\
$\quad$ Dummy variable 1 & -.01 & .01 & \\
$\quad$ Dummy variable 2 & .03 & .02 & \\
PTSD symptoms & .01 & .07 & .00 \\
PTSD symptoms (quadratic) & -.02 & -.36 & .01 \\
Interactions of Condition and Abuse, and Condition & & & .02 \\
and PTSD & & & \\
Condition (dummy 1) * PTSD & -.04 & -.07 & \\
Condition (dummy 2) * PTSD & -.01 & -.52 & \\
$\quad$ Condition (dummy 1) * PTSD (quadratic) & -.06 & -.08 & \\
$\quad$ Condition (dummy 2) PTSD (quadratic) & .02 & .58 & \\
Psych Abuse * Condition (dummy 1) & -.03 & .09 & \\
Psych Abuse * Condition (dummy 2) & -.11 & -.07 & \\
Interactions of Abuse and PTSD & & & \\
Psych Abuse * PTSD & -.02 & .04 & \\
Psych Abuse * PTSD (quadratic) & -.08 & .05 & \\
\hline
\end{tabular}


Table 47. Regression Coefficients predicting 15 min Cortisol Ratio as a Function of Sexual Abuse, PTSD Symptoms and Stressor Challenge

\begin{tabular}{|c|c|c|c|}
\hline & $r$ & $\bar{B}$ & $R_{\text {change }}^{2}$ \\
\hline \multicolumn{4}{|l|}{ Ratio of 15 min post-challenge/baseline } \\
\hline Sexual abuse & -.13 & -.13 & .02 \\
\hline Condition & & & .01 \\
\hline Dummy variable 1 & .12 & .11 & \\
\hline Dummy variable 2 & -.04 & .02 & \\
\hline PTSD symptoms & -.07 & -.05 & .00 \\
\hline PTSD symptoms (quadratic) & -.11 & -.57 & .02 \\
\hline $\begin{array}{l}\text { Interactions of Condition and Abuse, and Condition } \\
\text { and PTSD } \\
\text { Condition (dummy 1) * PTSD }\end{array}$ & -.12 & .46 & .06 \\
\hline Condition (dummy 2 ) * PTSD & .15 & -.09 & \\
\hline Condition (dummy 1 ) $*$ PTSD (quadratic) & -.05 & -.65 & \\
\hline Condition (dummy 2) * PTSD (quadratic) & .10 & .35 & \\
\hline Sexual Abuse $*$ Condition (dummy 1 ) & -.09 & -.13 & \\
\hline Sexual Abuse $*$ Condition (dummy 2) & .02 & .03 & \\
\hline Interactions of Abuse and PTSD & & & .01 \\
\hline Sexual Abuse * PTSD & .03 & .32 & \\
\hline Sexual Abuse * PTSD (quadratic) & -.06 & -.21 & \\
\hline
\end{tabular}


Table 48. Regression Coefficients predicting 30 min Cortisol Ratio as a Function of Sexual Abuse, PTSD Symptoms and Stressor Challenge

\begin{tabular}{|c|c|c|c|}
\hline & $r$ & $\bar{B}$ & $R_{\text {change }}^{2}$ \\
\hline \multicolumn{4}{|l|}{ Ratio of 30 min post-challenge/baseline } \\
\hline Sexual abuse & .08 & .08 & .01 \\
\hline Condition & & & .00 \\
\hline Dummy variable 1 & -.00 & .04 & \\
\hline Dummy variable 2 & .03 & .04 & \\
\hline PTSD symptoms & .01 & -.00 & .00 \\
\hline PTSD symptoms (quadratic) & -.02 & -.33 & .01 \\
\hline $\begin{array}{l}\text { Interactions of Condition and Abuse, and Condition } \\
\text { and PTSD } \\
\quad \text { Condition (dummy } 1 \text { ) * PTSD }\end{array}$ & -.04 & -.01 & .04 \\
\hline Condition (dummy 2) * PTSD & -.01 & -.52 & \\
\hline Condition (dummy 1) * PTSD (quadratic) & -.05 & -.15 & \\
\hline Condition (dummy 2) * PTSD (quadratic) & .02 & .42 & \\
\hline Sexual Abuse $*$ Condition (dummy 1 ) & -.02 & -.05 & \\
\hline Sexual Abuse $*$ Condition (dummy 2) & .14 & .20 & \\
\hline Interactions of Abuse and PTSD & & & .02 \\
\hline Sexual Abuse * PTSD & -.01 & -.72 & \\
\hline Sexual Abuse * PTSD (quadratic) & .03 & .89 & \\
\hline
\end{tabular}


Table 49. Regression Coefficients predicting 15 min Cortisol Ratio as a Function of Physical Abuse, PTSD Symptoms and Stressor Challenge

\begin{tabular}{|c|c|c|c|}
\hline & $\bar{r}$ & $\bar{B}$ & $R_{\text {change }}^{2}$ \\
\hline \multicolumn{4}{|l|}{ Ratio of $15 \mathrm{~min}$ post-challenge/baseline } \\
\hline Physical abuse & -.01 & -.01 & .00 \\
\hline Condition & & & .02 \\
\hline Dummy variable 1 & .12 & .14 & \\
\hline Dummy variable 2 & -.04 & .03 & \\
\hline PTSD symptoms & -.07 & -.07 & .01 \\
\hline PTSD symptoms (quadratic) & -.11 & -.53 & .02 \\
\hline $\begin{array}{l}\text { Interactions of Condition and Abuse, and Condition } \\
\text { and PTSD }\end{array}$ & & & .05 \\
\hline Condition (dummy 1) * PTSD & -.12 & .28 & \\
\hline Condition (dummy 2) * PTSD & .15 & -.09 & \\
\hline Condition (dummy 1) * PTSD (quadratic) & -.05 & -.51 & \\
\hline Condition (dummy 2) * PTSD (quadratic) & .10 & .36 & \\
\hline Physical Abuse $*$ Condition (dummy 1 ) & .06 & .16 & \\
\hline Physical Abuse * Condition (dummy 2) & .01 & .00 & \\
\hline Interactions of Abuse and PTSD & & & .00 \\
\hline Physical Abuse * PTSD & -.03 & .21 & \\
\hline Physical Abuse * PTSD (quadratic) & -.05 & -.37 & \\
\hline
\end{tabular}


Table 50. Regression Coefficients predicting 30 min Cortisol Ratio as a Function of Physical Abuse, PTSD Symptoms and Stressor Challenge

\begin{tabular}{llll}
\hline & $r$ & $B$ & $R^{2}{ }_{\text {change }}$ \\
\hline Ratio of 30 min post-challenge/baseline & -.03 & -.03 & .00 \\
Physical abuse & & & .00 \\
Condition & -.00 & .01 & \\
$\quad$ Dummy variable 1 & .03 & .03 & \\
$\quad$ Dummy variable 2 & .01 & .02 & .00 \\
PTSD symptoms & -.02 & -.36 & .01 \\
PTSD symptoms (quadratic) & & & .02 \\
Interactions of Condition and Abuse, and Condition & -.04 & -.04 & \\
and PTSD & -.01 & -.59 & \\
Condition (dummy 1) * PTSD & -.05 & -.12 & \\
Condition (dummy 2) * PTSD & .02 & .62 & \\
Condition (dummy 1) * PTSD (quadratic) & -.01 & .01 & \\
Condition (dummy 2) * PTSD (quadratic) & -.04 & -.07 & \\
Physical Abuse * Condition (dummy 1) & & & \\
Physical Abuse * Condition (dummy 2) & .01 & .24 & \\
Interactions of Abuse and PTSD & -.03 & \\
Physical Abuse * PTSD & & & \\
Physical Abuse * PTSD (quadratic) & & & \\
\hline
\end{tabular}

Discussion

As in Study 1, abuse was associated with well-being, in that having experienced abuse within an intimate relationship was correlated with greater symptoms of depression 
and PTSD. As well, as found in the initial study, abusive experiences were related to the use of potentially ineffective coping strategies. Emotional approach coping, which consists of the more adaptive strategies of processing and expressing emotions, was unrelated to abuse.

Consistent with Study 1, PTSD and depression were associated with the more frequent use of ineffective coping strategies. Specifically, the use of avoidant and emotion-focused styles of coping was associated with decreased well-being. As well, emotional approach coping was associated with fewer symptoms of depression. Similarly, previous studies found that emotional approach is correlated with a number of positive outcomes, such as lower anxiety and depression, and greater life satisfaction and self-esteem (Stanton et al., 1994; Stanton et al., 2000a). Emotion-focused coping consistently emerged as a mediator in the association between abuse and symptoms of psychological distress, while avoidant coping was also a mediator in certain cases. However, these methods of coping did not differ from one another in terms of their relative effects on the relationship between abuse and well-being. Since these variables were highly correlated, it is possible that they are very similar to one another and therefore it may be difficult to determine whether their effects vary. Alternatively, it is possible that although they are separate constructs, emotion-focused and avoidant coping exert their own similar, yet independent effects on well-being. Thus, although the data are correlational, and therefore do not allow for causal interpretations, the possibility exists that women in abusive relationships may utilize ineffective means of dealing with relationship conflict, which then lead to symptoms of depression and PTSD. 
Although in Study 1, problem-focused coping was unrelated to all three types of abuse, in Study 2, this dimension of coping unexpectedly showed a positive correlation with physical assault. It is unclear why this disparity in results occurred; however, this correlation was very small and may have been specific to the sample used in this study. To the extent that problem-focused coping is related to emotional approach coping, which involves the active use of emotional processing and expression to deal with stressful events, it is perhaps not surprising that emotional approach coping was also not related to abuse in the present study. Indeed, previous studies found that emotional approach and problem-focused coping involve similar mechanisms and are therefore correlated with one another (Stanton et al., 2000b). In the present study, problem-focused coping and emotional approach were significantly correlated, although the correlation was not necessarily high enough to conclude that they represent the same construct.

Women in abusive relationships appeared to have altered appraisals of abusive behaviours. Specifically, women who experienced sexual coercion in their relationships were less likely to view abusive behaviours as malevolent. Likewise, a previous study found that undergraduate women in physically abusive relationships rated restrictive and domineering behaviours as less malevolent compared to those not in abusive relationships (Ehrensaft \& Vivian, 1999). It has been suggested that not defining behaviours as abusive may be a strategy to blunt the emotional distress associated with such an experience, or may provide some rationalization for enduring abusive behaviour (Ehrensaft \& Vivian, 1999). This finding contrasts with the work of Wagner and Mongan (1998), however, in which women in abusive relationships appraised emotionally abusive behaviours as more offensive, but not those behaviours considered physically abusive, in comparison to non- 
abused women. However, this study was complicated by the fact that abuse was assessed by a single item asking whether the individual had ever been abused, such that it was not possible to differentiate between current and previous abuse, nor was it possible to disentangle effects of abuse experienced in an intimate relationship versus that experienced in childhood. Appraisals of abusive behaviours, however, were not associated with well-being in the present study and thus did not mediate the effects of abuse on symptoms of depression and PTSD.

Since previous researchers had indicated that appraisals might influence the coping strategy used (e.g., Folkman \& Lazarus, 1980), the effect of appraisals in mediating the relationship between abuse and coping was also assessed. Indeed, coping and appraisals were related in this study. Appraisals of control over events were inversely related to the use of emotion-focused and avoidant coping. In addition, increased avoidant coping was associated with the perception of behaviours from the CTS-R as being less abusive. It was found that appraisals of control mediated the relationship between psychological aggression and use of ineffective coping styles. That is, psychological maltreatment affected the use of emotion-focused and avoidant coping, in part due to appraisals of having less control over events.

Psychological aggression was associated with a perceived lack of control over the outcome of ambiguous events in dating relationships. Perceiving less control over the outcome of events was also associated with greater symptoms of depression and PTSD. Furthermore, perceptions of control mediated the effects of psychological abuse on symptoms of depression and PTSD. Since all of the behaviours in this scale were instances of potential psychological maltreatment, rather than physical or sexual, it is 
possible that some women, regardless of past experiences of abuse, have more difficulty recognizing the potential malevolent nature of behaviours that are not overtly aggressive. It is important to further assess this possibility, especially since psychological abuse may be associated with symptoms of psychological distress above and beyond the effects of physical or sexual abuse (e.g., Katz \& Arias, 1999). As well, many women experience emotional abuse concurrently with other more overt forms of aggression (Coker et al., 2000).

In the present study, cortisol levels did not vary as a function of either abuse or challenge condition. That is, being in an abusive relationship did not moderate cortisol levels in response to a challenge, nor did the presence of PTSD symptoms affect this relationship. Furthermore, the particular image participants were asked to speak about (abuse scene, accident scene, or a neutral image) did not influence cortisol levels. This contrasts with a previous study (Matheson, 2003), which demonstrated that women in abusive relationships, especially those with PTSD symptoms, had an increased cortisol response to a challenge when first primed to recall traumatic events. It is not clear why abuse was unrelated to cortisol in the present study. It is possible that a ceiling effect occurred for the speech task, such that women experienced high levels of stress regardless of their past experiences of abuse, or the specific group that they had been assigned to. Indeed, it has been proposed that contexts characterized by social evaluation (e.g., when one is speaking to an audience or being videotaped) may be particularly likely to elicit increases of cortisol due to the perceived threat that aspects of one's self-identity may be judged negatively by others (Dickerson \& Kemeny, 2004; Dickerson, Gruenewald, \& Kemeny, 2004). Thus, the effects of abuse may have been obfuscated by 
the public speaking component of the test situation. Alternatively, it is possible that the speech task was not a sufficient stressor to cause alterations in cortisol levels.

\section{General Discussion}

The aim of this research was to assess experiences of abuse among women in dating relationships, the effects of such experiences on well-being and neuroendocrine activity, and the potential mediating effects of support, coping, and appraisals. Unlike much of the previous research on battered women who had been experiencing abuse for a protracted period of time, the present study involved women in the earlier stages of dating relationships who were without certain constraints that would otherwise keep them in the relationship (e.g., children, financial dependence). It is important to study the factors that are associated with remaining in an abusive dating relationship, especially since it is known that abuse is not uncommon in dating relationships, and may begin early in a relationship and increase over time (Arias et al., 1987; DeKeseredy \& Kelly, 1993; Kuffel \& Katz, 2002; Makepeace, 1981; White \& Koss, 1991). In fact, abuse experienced at this stage may be more frequent and of greater severity than that experienced later in life (e.g., Statistics Canada, 2000, 2005; Yoshihama \& Horrocks, 2003).

Experiences of maltreatment were related to increased symptoms of psychological distress in previous studies (e.g., Golding, 1999; Stein \& Kennedy, 2001; Street \& Arias, 2001). Accordingly, in the present research, experiences of abuse were associated with increased symptoms of depression and PTSD. As well, variations in social support, appraisals, and coping were associated with experiences of abuse within intimate relationships. Appraisals of control and use of emotion-focused coping strategies mediated the effects of abuse on well-being. Consistent with expectations, support from 
one's partner also functioned as a mediator. Although the present data are correlational in nature, they are consistent with the perspective that experiences of abuse may lead to altered coping styles and appraisals, as well as changes in levels of social support, which may then render women more vulnerable to developing symptoms of psychological distress. In line with previous research concerning the effects of appraisals in the coping process (e.g., Folkman \& Lazarus, 1980), it was found that the relationship between psychological abuse and ineffective coping styles was mediated by appraisals of control. However, in contrast to previous research that showed variations in neuroendocrine functioning in relation to abuse and symptoms of PTSD (e.g., Matheson, 2003), in the present studies, cortisol was not related to either of the variables.

Relative to non-abused women, those in abusive relationships reported greater use of emotion-focused coping. This is consistent with previous research which demonstrated that such strategies are frequently used by women in abusive relationships, and that emotion-focused coping may actually be adaptive, at least in the short term, for dealing with the harmful emotional effects of abuse (Matheson et al., 2007; Rosen, 1996). In contrast, abuse was not associated with the use of problem-focused coping. Similarly, abuse was unrelated to the use of adaptive, emotional approach coping strategies, which were previously reported to be related to problem-focused coping (Stanton et al., 2000b). To the extent that abused women refrain from using active coping strategies, this may actually be adaptive, especially in situations in which individuals perceive a lack of control over their experiences of abuse. Indeed, perceived controllability over the stressor is a critical factor in predicting which strategy will be used: whereas problem-focused coping is more common in situations that are appraised as being controllable, emotion- 
focused strategies are used more often in situations that are perceived as being out of one's control (Bijttebier et al., 2001; Folkman \& Lazarus, 1980). In fact, it has been suggested that in situations of abuse that are appraised as uncontrollable, attempts to actively address the problem may be met in vain (Zakowski et al., 2001). In line with this, it was previously found that a negative correlation between problem-focused coping and depressive symptoms was particularly strong among women in psychologically abusive relationships. This association was not, however, pronounced among those experiencing physical abuse, indicating that use of problem-focused coping may not be adaptive in situations of more overt maltreatment (Matheson et al., 2007). It is possible that women perceive having less control over such events compared to psychological abuse, and attempts to actively cope with physical aggression may be ineffective or even harmful.

In the present study, women in psychologically abusive relationships reported less control over the outcome of behaviours that may be considered abusive. However, physical and sexual abuse were not associated with appraisals of control. To the extent that individuals choose coping strategies based on the controllability of the situation, it would be expected that physically and sexually abused women would appraise relationship conflicts as being less controllable than non-abused women. It must be noted that in the present study, participants were not asked directly about their own feelings of control; rather, these feelings were inferred from their responses to the experimental stimuli (i.e., the situations of potential maltreatment given in the AASQ) in order to examine general perceptions of events. It is possible that these women did not view the behaviours on the Appraisals of Ambiguous Events Questionnaire as instances of abuse. 
Since the items indicated only potential experiences of psychological maltreatment, it is conceivable that women experiencing more overt types of abuse (i.e., physical or sexual) would not consider these behaviours to be abusive. However, when asked to appraise the items from the Conflict Tactics Scale, women who experienced sexual coercion in their relationships were less likely to view abusive behaviours of all types (sexual, physical, and psychological) as malevolent. To the extent that they experienced such behaviours in their own relationship, failing to define malevolent behaviours as abusive may serve as a strategy to attenuate the emotional distress associated with such behaviours.

Alternatively, failing to perceive such behaviours as instances of abuse may provide some justification for remaining in the abusive relationship (Ehrensaft \& Vivian, 1999).

Although associated with actual experiences of maltreatment, appraisals of abusive behaviour were not associated with depressive or PTSD symptoms, and thus did not exhibit a mediating role in the relationship between abuse and well-being.

Coping and appraisals are intricately related to one another, in that appraisals of events influence the choice of coping strategy used, which in turn can lead to reappraisals. It is recognized that although individuals might have characteristic ways of appraising and coping with events, the specific strategy used may depend upon aspects of the situation (Heszen-Niejodek, 1997; Zakowski et al., 2001). To the extent that coping and appraisals can vary across circumstances, it is important to recognize that responses in the present survey may not be stable over time. For instance, if an individual had a disagreement with her partner the day before participating in the survey, her responses may have been different than if her and her partner had a good day or had recently resolved a conflict. Indeed, previous research has shown that coping and appraisals vary 
even across stages of a single stressful event. For example, college students reported variations in appraisal-related emotions (e.g., threat, challenge, harm) and coping between before writing an exam and waiting for their grade (Carver \& Scheier, 1994; Folkman \& Lazarus, 1985) and across phases of a sports competition (Gaudreau, Blondin, \& Lapierre, 2002). Furthermore, in situations of abuse, coping strategies may change over time depending on situational factors. For example, attributions of blame for abusive behaviours were more likely to shift from the self to the partner when violence increased in severity (Miller \& Porter, 1983). The dynamic nature of coping and appraisals points to the utility of longitudinal research to determine the interactions between these variables over time in abusive relationships.

Not unexpectedly, in both studies, abuse was associated with lower levels of support from the abusive partner. However, there were no differences in reported support from friends or from parents. Similarly, in a previous study, abused women reported lower support from their partner compared to non-abused women, but there were no differences in social support from other sources (Carlson et al., 2002). However, others (e.g., Smith et al., 2002) have found that abused women report less support from friends and family. Inconsistencies between studies may result from the method of measuring abuse, or the population examined (e.g., dating relationships versus longer-term). As these women were in the earlier stages of their relationships, most of the abuse reported in the present study was not of a severe nature, according to the classifications of items as severe by Straus and colleagues (1996). It has been suggested in that women experiencing severe intimate partner abuse may be intentionally isolated from social contacts by their partner, or that friends and family may be less willing or able to provide 
support in cases of high levels of violence (Dobash \& Dobash, 1998; Waldrop \& Resick, 2004). It is possible that compared to battered women in longer-term abusive relationships, those experiencing less severe forms of abuse early in their dating relationships may still be able to maintain strong social networks, particularly when in a university setting where they presumably have frequent contact with others.

Across both studies, women in abusive relationships did not differ from nonabused women in previous experiences of assault. This contrasts with the findings of some other researchers, who have indicated that abused women are more likely to have experienced assault earlier in life (e.g., Banyard et al., 2000; Matheson, Skomorovsky, Fiocco, \& Anisman, 2007). However, although the difference was not statistically significant, it must be noted that a higher percentage of abused, compared to non-abused women did experience assault. It is possible that the low sample size may have precluded this difference from reaching statistical significance. In addition, the percentages reported here are of overall past experiences as measured by the TLEQ, and various experiences are combined in creating broad subscales according to types of previous trauma. It remains a possibility that women in abusive relationships experienced more of certain specific types of assault (e.g., childhood sexual abuse) than others (e.g., robbery). However, this section of the report was not to provide detail about specific past experiences of abused women, but mainly to give a general overview of experiences of previous trauma.

Mediational analyses were used in the present studies to examine the factors involved in the relationship between abuse and well-being. The nature of a mediation model is that it is assumed that the independent variable leads to changes in the mediator, 
which in turn results in changes in the dependent variable (Baron \& Kenny, 1986). In the present research, it was proposed that abuse led to changes in the mediating variables of interest, which then accounted for at least part of the effect of abuse on symptoms of depression and PTSD. According to this view, coping, social support and appraisals were considered similar to state-like variables, which may be altered depending on one's situation and the stressors one encounters, as opposed to trait-like variables, which are relatively constant across situations. This is in accordance with much of the past research. For example, Kaniasty and Norris (1993) suggested that stressful experiences can negatively affect perceptions of social support. As well, it has been suggested that although individuals may have a limited repertoire of coping strategies at their disposal, the particular strategies used are largely dependent upon the situation (Lerner \& Kennedy, 2000; Zakowski et al., 2001). However, as mentioned in the introduction, many researchers consider coping to be more analogous to a trait-like variable. Along this line, most individuals have a characteristic style of coping that they tend to favour across situations, and that is relatively independent of the stressor encountered. To the extent that coping represents a trait-like quality, it may be advantageous to also assess general styles of coping with stressful events. Indeed, some differences have been found between general and specific styles of coping. For example, Matheson et al. (2007) found that women indicated using all types of coping strategies more often when asked about their general styles of coping, relative to coping with a relationship conflict, indicating that general coping style is not necessarily indicative of the type of coping used to deal with specific events. 
Although mediating effects of coping, appraisals, and support were found in the samples under study, it is possible that more complex models may be present in the population. For example, social support may bolster one's ability to utilize adaptive, problem-focused styles of coping in the face of stress, which may in turn have positive effects on well-being, or it may influence coping styles indirectly through its effects on appraisals. Indeed, it has been suggested that social support precedes coping (Lazarus \& Folkman, 1984), and may function as a type of "coping assistance" (Thoits, 1986) such that a stressor is perceived as less threatening and therefore more likely to be within the realm of what an individual can cope with effectively. Indeed, in the second study, emotion-focused and avoidant coping were associated with appraisals of decreased control over the outcome of potentially abusive behaviours, in line with previous findings concerning the utility of emotion-focused strategies for dealing with uncontrollable events (Bijttebier et al., 2001). As well, avoidant coping was associated with appraisals of abusive behaviours as being less malevolent. That is, women who tended to use avoidant coping strategies to deal with their experiences of relationship conflict were less likely to view aggressive behaviours as instances of abuse. Although the sample sizes in the present studies were too low to perform more complex analyses, it may ultimately be instructive to assess the abuse-depression and abuse-PTSD connection through more sophisticated and informative statistical techniques (e.g., structural equation modelling).

Alterations in neuroendocrine activity have previously been found following experiences of trauma. For example, depressed adult women with a history of childhood abuse exhibited a blunted ACTH response to exogenous CRH administration (Heim et al., 2001), but an exaggerated ACTH response to a psychosocial laboratory stressor 
(Heim et al., 2002). It was suggested that although traumatic experiences may reduce activity of the HPA axis, other areas of the brain may be able to overcome this downregulation. Furthermore, these findings highlight the fact that it may be difficult to detect subtle changes in HPA functioning among individuals exposed to severe or protracted stressors without the presence of a challenge. Thus, challenge stimuli were given to participants in the present study in order to examine cortisol changes. However, contrary to expectation, there were no differences between abused and non-abused women in baseline cortisol levels throughout both studies. In addition, neither of the studies revealed a moderating effect of abuse or symptoms of PTSD in predicting cortisol ratios following the challenge. In Study 1, the lack of effect may have occurred due to the fact that the participants were not given any reminder stimuli prior to completing the challenge. Without recalling previous traumatic experiences, it is possible that the stressor was not sufficient to induce neuroendocrine alterations. However, even though participants in Study 2 were reminded of previous stressors prior to the challenge (i.e., by filling out information regarding traumatic experiences across their lifetime and conflicts within their intimate relationship), no differences in cortisol release were found. These findings contrast with a previous study (Matheson, 2003), which demonstrated that abused women exhibited heightened neuroendocrine activity following a challenge, and this was particularly pronounced among those with high levels of PTSD symptoms. However, in that study, women watched a video depicting a situation in which a woman had been abused, which may have been a more significant stressor than those used in the present study. In another study, although ACTH levels were elevated among depressed women with a history of childhood abuse following a laboratory stressor, cortisol 
responses to a psychosocial stressor were not affected by adult trauma or an interaction of adult and childhood events; instead, cortisol was inversely related to the severity of daily hassles (Heim et al., 2002). Thus, although changes in one component of the HPA axis may be evident following exposure to a stressor, alterations in other components might be subtler and might only be seen in response to other types of events. In fact, the authors suggested that traumatic experiences and milder daily stressors may have differential effects at the level of the adrenal gland and pituitary (Heim et al., 2002). It may be the case that the stressors given in the present studies were not sufficient to induce a cortisol response. Neuroendocrine alterations might only be evident in response to certain events or when an individual is sufficiently involved in a task. It is possible that an empathic response is more likely when watching someone else speak about their experiences (as happened in the video used in the Matheson [2003] study) as opposed to viewing them in a photograph. In addition to the lack of effects of the stressor, it may be the case that the abuse or PTSD symptoms reported by participants in the present research were not of sufficient severity to lead to cortisol alterations.

Although it was of interest in the present research to assess neuroendocrine response to a challenge, it may be useful in future research to also examine diurnal alterations in cortisol. Previous studies indicated that patterns of cortisol release throughout the day varied between abused and non-abused women, and this was often dependent on the level of depressive or PTSD symptoms experienced. For example, previous experience of abuse was associated with low morning plasma cortisol levels regardless of the symptoms of psychological distress reported (Seedat et al., 2003), whereas among women currently experiencing abuse, morning cortisol levels were low 
among those with symptoms of depression and PTSD (Griffin et al., 2005). However, another study found that abused women exhibited higher evening, but not morning, levels of cortisol, irrespective of the level of depression or PTSD (Pico-Alfonso et al., 2004). Clearly, the profile of cortisol changes in abused women depends upon a number of factors. In addition to the levels of symptomatology experienced, factors such as time since the last abusive encounter and duration of the abuse, in addition to the level of psychological distress experienced, are likely to affect cortisol levels. However, diurnal cortisol patterns, when examined with consideration of such factors, may be a more sensitive index of neuroendocrine alterations associated with abuse than cortisol response to a challenge.

There are several limitations to the present research. As with any retrospective, cross-sectional study, conclusions cannot be drawn concerning the presence of causal relationships. That is, as already indicated, it cannot be stated with certainty that abuse caused individuals to utilize ineffective coping strategies or to be less capable of maintaining social support systems, which then caused the development of symptoms of depression and PTSD. Some evidence of the direct effects of abuse on coping is supported by the finding that once women leave abusive relationships, the use of emotion-focused coping strategies decreases over time, whereas the use of problemfocused strategies increases (Lerner \& Kennedy, 2000); however, coping has also been considered a trait-like variable which remains relatively consistent across situations, and so the possibility that abuse can lead to changes in coping strategies endorsed needs to be further assessed. 
Furthermore, it must be emphasized that since the present study was correlational, the relationships between abuse, well-being, and the mediating variables examined are not necessarily unidirectional. Although the assumption is made that symptoms of depression and PTSD are outcomes of abuse and maladaptive appraisals and coping strategies, it is equally possible that symptoms of psychological distress can influence these variables. For example, the cognitive and affective disturbances associated with depressive disorder may lead to altered perceptions of events. As indicated in the introduction, although abuse may lead to symptoms of psychological distress, such symptoms may also contribute to the maintenance of abuse. For example, the cognitive and motivational deficits associated with depression may result in reduced awareness that abuse is occurring, or a lower likelihood of seeking treatment or leaving the relationship, in effect limiting one's ability to deal with relationship conflicts (Clements \& Sawhney, 2000). Certain coping styles may in fact be characteristic of disorders; indeed, one of the core symptoms of PTSD is avoidance (American Psychiatric Association, 1994). It has been suggested that coping strategies and symptoms of depression may operate in a reciprocal fashion, whereby symptoms increase as a result of ineffective coping, yet those symptoms also lead to the increased use of ineffective coping strategies (Clements \& Sawhney, 2000). In addition, high levels of psychological distress may impact upon one's level of social support by limiting one's capability or motivation to develop and maintain strong social networks. Only with the use of a longitudinal design would it be possible to confirm the direction and causality of the relationships proposed in this report. In this way, it could be determined whether coping, appraisals, and social support are altered when women enter into abusive relationships, whether symptoms of depression and 
PTSD result from these alterations, and also whether coping styles, appraisals and levels of social support change once individuals leave an unhealthy relationship.

The present studies may have also been limited to some extent by the measure of abusive behaviour utilised. The use of a self-report scale to recall past events may be problematic, since individuals may have difficulty remembering accurately the details of events that occurred during times of stress (King, King, Foy, \& Gudanowski, 1996; King, King, Gudanowski, \& Vreven, 1995). Although participants were asked about their experiences with intimate partner violence within the previous month, it remains a possibility that problems in recall or biases in perception occurred, particularly among women experiencing severe abuse. Furthermore, although the authors of the CTS attempted to make the scale comprehensive in its scope, the scale has been criticised for excluding many behaviours that may be considered physically, sexually, or psychologically abusive (e.g., stalking; DeKeseredy \& Schwartz, 1998b). The CTS considers tactics used to resolve conflicts, disregarding the fact that individuals in relationships (men in particular) sometimes make use of control-instigated violence, or violence outside of disagreements or conflicts that is used to control the other (e.g., with the intention to dominate or punish; Ellis, 1995). As well, the CTS fails to consider the context, meaning and motives of abusive behaviours, as well as the psychological effects of such behaviours (DeKeseredy, Saunders, Schwartz, Alvi, 1997; DeKeseredy \& Schwartz, 1998a). For example, it is unclear from the wording of the items whether the behaviours reported were done in self-defence or were actually instigated by the partner. Furthermore, the CTS does not include items on certain aggressive behaviours, such as dominance and isolation, which were related to extent of PTSD symptoms among abused 
women (Cascardi et al., 1999). Thus, it is possible that some women who were experiencing abuse were not captured in the survey. Alternatively, self-report scales on abuse may falsely classify women as abused if they answer items that they do not necessarily consider malevolent (see Hamby, 2005). Although the CTS is among the most widely used scales to assess aggression by an intimate partner, it may be of benefit to supplement it with questions on context, with other validated scales, and/or with openended questions, in order to provide greater opportunity to disclose abusive events, and to allow researchers to assess more accurately the extent of aggression in dating relationships.

Finally, there may be limitations to the generalizability of the present studies. The sample was taken from the pool of undergraduate students, primarily in their first year of studies, who were taking an introductory course in psychology. Thus, caution must be exercised when attempting to generalize from this study to all females in dating relationships. In addition, across both studies, the most severe abusive items were generally not endorsed. This is consistent with a previous study using a university sample (DeKeseredy \& Schwartz, 1998), which showed that the less lethal and less severe forms of violence were reported most often. Since the participants did not endorse the majority of more severe abuse items, the results of this study cannot be generalized to women experiencing abuse of a more severe and protracted nature, although this was not the intent, since the focus of the study was on younger women in dating relationships. However, the relationships found in this study may be indicative of the psychological alterations that may occur among women in the earlier stages of such relationships. 
Taken together, the results of the present research suggest that women in abusive dating relationships experience heightened levels of psychological distress, in terms of depression and PTSD symptoms. The effects of abusive experiences, however, can be mediated by certain factors, such that not all women experiencing abuse will develop symptoms. It remains to be seen whether neuroendocrine alterations would be evident in these women throughout the day or in response to a different type of stressor.

Nonetheless, the findings point to the fact that negative patterns of dealing with conflict may begin to form in the early stages of dating relationships, and abusive experiences may be prevalent even in these early stages, potentially leading to psychological disorder. It is important, therefore, to recognize the factors associated with abuse so that appropriate educational and intervention strategies can be developed at an early stage before abuse escalates and before symptoms of psychological distress become evident. 


\section{References}

Ackard, D. M., Neumark-Sztainer, D., \& Hannan, P. (2003). Dating violence among a nationally representative sample of adolescent girls and boys: Associations with behavioral and mental health. Journal of Gender Specific Medicine, 6, 39-48.

Akechi, T., Okamura, H., Yamawaki, S., \& Uchitomi, Y. (1998). Predictors of patients' mental adjustment to cancer: patient characteristics and social support. British Journal of Cancer, 77, 2381-2385.

Andrews, B., \& Brewin, C. R. (1990). Attributions of blame for marital violence: A study of antecedents and consequences. Journal of Marriage and the Family, 52, 757767.

Angst, J., \& Merikangas, K. (1997). The depressive spectrum: Diagnostic classification and course. Journal of Affective Disorders, 45, 31-39.

American Psychiatric Association (1994). Diagnostic and statistical manual of mental disorders $\left(4^{\text {th }}\right.$ ed.). Washington, DC: Author.

Anisman, H. \& Matheson, K. (2005) Anhedonia and depression: Caveats concerning animal models. Neuroscience and Biobehavioral Reviews, 29, 525-546.

Arato, M., Banki, C. M., Bisette, G., \& Nemeroff, C. B. (1989). Elevated CSF CRF in suicide victims. Biological Psychiatry, 25, 355-359.

Arborelius, L., Owens, M. J., Plotsky, P. M., \& Nemeroff, C. B. (1999). The role of corticotropin-releasing factor in depression and anxiety disorders. Journal of Endocrinology, 160, 1-12. 
Arias, I., Lyons, C. M., \& Street, A. E. (1997). Individual and marital consequences of victimization: Moderating effects of relationship efficacy and social support. Journal of Family Violence, 12, 193-210.

Arias, I., \& Pape, K. T. (1999). Psychological abuse: Implications for adjustment and commitment to leave violent partners. Violence and Victims, 14, 55-67.

Arias, I., Samios, M., \& O’Leary, K. D. (1987). Prevalence and correlates of physical aggression during courtship. Journal of Interpersonal Violence, 2, 82-90.

Arriaga, X. B. (2002). Joking violence among highly committed couples. Journal of Interpersonal Violence, 17, 591-610.

Astin, M. C., Lawrence, K. J., \& Foy, D. W. (1993). Posttraumatic stress disorder among battered women: Risk and resiliency factors. Violence and Victims, 8, 17-27.

Austenfeld, J. L., \& Stanton, A. L. (2004). Coping through emotional approach: A new look at emotion, coping, and health-related outcomes. Journal of Personality, 72 , $1335-1364$.

Baker, D. G., West, S. A., Nicholson, W. E., Ekhator, N. N., Kasckow, J. W., Hill, K. K., Bruce, A. B., Orth, D. N., \& Geracioti, T. D. Jr. (1999). Serial CSF corticotropinreleasing hormone levels and adrenocortical activity in combat veterans with posttraumatic stress disorder. American Journal of Psychiatry, 156, 585-588.

Banyard, V. L., Arnold, S., \& Smith, J. (2000). Childhood sexual abuse and dating experiences of undergraduate women. Child Maltreatment: Journal of the American Professional Society on the Abuse of Children, 5, 39-48. 
Barnett, O. W., Martinez, T. E., \& Keyson, M. (1996). The relationship between violence, social support, and self-blame in battered women. Journal of Interpersonal Violence, 11, 221-233.

Baron, R. M., \& Kenny, D. A. (1986). The moderator-mediator variable distinction in social psychological research: Conceptual, strategic, and statistical considerations. Journal of Personality and Social Psychology, 51, 1173-1182.

Beach, S. R. H., Arias, I., \& O'Leary, K. D. (1986). The relationship of marital satisfaction and social support to depressive symptomatology. Journal of Psychopathology and Behavioral Assessment, 8, 305-316.

Beck, A. T. \& Beck, R. W. (1972). Screening depressed patients in family practice: A rapid technique. Postgraduate Medicine, 52, 81-85.

Bergman, B., \& Brismar, B. (1991). A 5-year follow-up study of 117 battered women. American Journal of Public Health, 81, 1486-1489.

Bernhard, L. A. (2000). Physical and sexual violence experienced by lesbian and heterosexual women. Violence Against Women, 6, 68-79.

Bijttebier, P., Vertommen, H., \& Vander Steene, G. (2001). Assessment of cognitive coping styles: A closer look at situation-response inventories. Clinical Psychology Review, 21, 85-104.

Billings, A. G. \& Moos, R. H (1984). Coping, stress, and social resources among adults with unipolar depression. Journal of Personality and Social Psychology, 46, $877-$ 891. 
Blalock, J. A., \& Joiner, T. E., Jr. (2000). Interaction of cognitive avoidance coping and stress in predicting depression/anxiety. Cognitive Therapy and Research, 24, 4765.

Bremner, J. D., Licinio, J., Darnell, A., Krystal, J. H., Owens, M. J., Southwick, S. M., Nemeroff, C. B., \& Charney, D. S. (1997). Elevated CSF corticotropin-releasing factor concentrations in posttraumatic stress disorder. American Journal of Psychiatry, 154, 624-629.

Breslau, N., Chilcoat, H. D., Kessler, R. C., \& Davis, G. C. (1999). Previous exposure to trauma and PTSD effects of subsequent trauma: Results from the Detroit area survey of trauma. American Journal of Psychiatry, 156, 902-907.

Brewin, C. R., Andrews, B., \& Valentine, J. D. (2000). Meta-analysis of risk factors for posttraumatic stress disorder in trauma-exposed adults. Journal of Consulting and Clinical Psychology, 68, 748-766.

Britton, D. R., Koob, G. F., Rivier, J., \& Vale, W. (1982). Intraventricular corticotropinreleasing factor enhances behavioral effects of novelty. Life Sciences, 31, 363367.

Broadhead, W. E., Blazer, D. G., George, L. K., \& Tse, C. K. (1990). Depression, disability days, and days lost from work in a prospective epidemiologic survey. Journal of the American Medical Association, 264, 2524-2528.

Brown, E. S., Rush, A. J., \& McEwen, B. S. (1999). Hippocampal remodeling and damage by corticosteriods: Implications for mood disorders. Neuropsychopharmacology, 21, 474-484. 
Calfa, G., Kademian, S., Ceschin, D., Vega, G., Rabinovich, G. A., \& Volosin, M. (2003). Characterization and functional significance of glucocorticoid receptors in patients with major depression: Modulation by antidepressant treatment. Psychoneuroendocrinology, 28, 687-701.

Campbell, J. C., \& Soeken, K. L. (1999). Forced sex and intimate partner violence: Effects on women's risk and women's health. Violence Against Women, 5, 10171035.

Carey, C. M., \& Mongeau, P. A. (1996). Communication and violence in courtship relationships. In D. D. Cahn \& S. A. Lloyd (Eds.), Family violence from a communication perspective (pp. 127-150), Thousand Oaks, CA: Sage Publications.

Carlson, B. E., McNutt, L. A., Choi, D. Y., \& Rose, I. M. (2002). Intimate partner abuse and mental health. The role of social support and other protective factors. Violence Against Women, 8, 720-745.

Carver, C. S., Scheier, M. F., \& Weintraub, J. K. (1989). Assessing coping strategies: A theoretical based approach. Journal of Personality and Social Psychology, 56, $267-283$.

Carver, C. S., \& Scheier, M. F. (1994). Situational coping and coping dispositions in a stressful transaction. Journal of Personality and Social Psychology, 66, 184-195.

Cascardi, M., O'Leary, K. D., \& Schlee, K. A. (1999). Co-occurrence and correlates of posttraumatic stress disorder and major depression in physically abused women. Journal of Family Violence, 14, 227-249. 
Casper, R. C., Swann, A. C., Stokes, P. E., Chang, S., Katz, M. M., \& Garver, D. (1987). Weight loss, cortisol levels, and dexamethasone suppression in major depressive disorder. Acta Psychiatrica Scandinavica, 75, 243-250.

Chang, E. C., \& Strunk, D. R. (1999). Dysphoria: Relations to appraisals, coping, and adjustment. Journal of Counseling Psychology, 46, 99-108.

Clements, C. M., Sabourin, C. M., \& Spiby, L. (2004). Dysphoria and hopelessness following battering: The role of perceived control, coping, and self-esteem. Journal of Family Violence, 19, 25-36.

Clements, C. M., \& Sawhney, D. K. (2000). Coping with domestic violence: Control attributions, dysphoria, and hopelessness. Journal of Traumatic Stress, 13, 219240.

Coker, A. L., McKeown, R. E., Sanderson, M., Davis, K. E., Valois, R. F., \& Huebner, E. S. (2000). Severe dating violence and quality of life among South Carolina high school students. American Journal of Preventive Medicine, 19, 220-227.

Coker, A. L., Smith, P. H., Bethea, L., King, M. R., \& McKeown, R. E. (2000). Physical health consequences of physical and psychological intimate partner violence. Archives of Family Medicine, 9, 451-457.

Coker, A. L., Smith, P. H., Thompson, M. P., McKeown, R. E., Bethea, L., \& Davis, K. E. (2002). Social support protects against the negative effects of partner violence on mental health. Journal of Women's Health and Gender Based Medicine, 11, $465-476$. 
Coker, A. L., Watkins, K. W., Smith, P. H., \& Brandt, H. M. (2003). Social support reduces the impact of partner violence on health: Application of structural equation models. Preventive Medicine, 37, 259-267.

Collins, L. M., Graham, J. W., \& Flaherty, B. P. (1998). An alternative framework for defining mediation. Multivariate Behavioral Research, 33, 295-312.

Compas, B. E., Connor, J., Osowiecki, D., \& Welch, A. (1997). Effortful and involuntary responses to stress: Implications for coping with chronic stress. In B. H. Gottlieb (Ed.). Coping with chronic stress (pp.105-130). New York, NY, US: Plenum Press.

Creamer, M., Bell, R., \& Failla, S. (2003). Psychometric properties of the Impact of Events Scale - Revised. Behaviour Research and Therapy, 41, 1489-1496.

Cutrona, C. E., \& Russell, D. W. (1987). The provisions of social relationships and adaptation to stress. Advances in Personal Relationships, 1, 37-67.

De Bellis, M. D., Gold, P., Geracioti, T. D., Listwak, S. J., \& Kling, M. A. (1993). Association of fluoxetine treatment with reductions in CSF concentrations of corticotropin-releasing hormone and arginine vasopressin in patients with major depression. American Journal of Psychiatry, 150, 656-657.

DeKeseredy, W. S., \& Kelly, K. (1993). The incidence and prevalence of woman abuse in Canadian university and college dating relationships. Canadian Journal of Sociology, 18, 137-159.

DeKeseredy, W. S., Saunders, D. G., Schwartz, M. D., \& Alvi, S. (1997). The meanings and motives for women's use of violence in Canadian college dating relationships: Results from a national survey. Sociological Spectrum, 17, 199-222. 
DeKeseredy, W. S., \& Schwartz, M. D. (1998a). Measuring the extent of woman abuse in intimate heterosexual relationships: A critique of the Conflict Tactics Scales. In U.S. Department of Justice Violence Against Women Grants Office Electronic Resources. Retrieved August 28, 2005, from http://www.vaw.umn.edu/research.asp.

DeKeseredy, W. S., \& Schwartz, M. D. (1998b). Woman abuse on campus. Results from the Canadian National Survey. Sage Publications: London.

De Souza, E. B. (1995). Corticotropin-releasing factor receptors: Physiology, pharmacology, biochemistry, and role in central nervous system and immune disorders. Psychoneuroendocrinology, 20, 789-819.

Dickerson, S. S., Gruenewald, T. L., \& Kemeny, M. E. (2004). When the social self is threatened: Shame, physiology, and health. Journal of Personality, 72, 11921216.

Dickerson, S. S., \& Kemeny, M. E. (2004). Acute stressors and cortisol responses: A theoretical integration and synthesis of laboratory research. Psychological Bulletin, 130, 355-391.

Dobash, R., \& Dobash, R. (1998). Violent men and violent contexts. In R. Dobash \& R. Dobash (Eds.), Rethinking violence against women (pp. 141-168). Thousand Oaks, CA: Sage.

Dunham, K., \& Senn, C. (2000). Minimizing negative experiences: Women's disclosure of partner abuse. Journal of Interpersonal Violence, 15, 251-261.

Efron, B. (1987). Better bootstrap confidence intervals. Journal of the American Statistical Association, 82, 171-185. 
Ehlert, U., Gaab, J., \& Heinrichs, M. (2001). Psychoneuroendocrinological contributions to the etiology of depression, posttraumatic stress disorder, and stress-related bodily disorders: The role of the hypothalamus-pituitary-adrenal axis. Biological Psychiatry, 57, 141-152.

Ehrensaft, M. K., \& Vivian, D. (1999). Is partner aggression related to appraisals of coercive control by a partner? Journal of Family Violence, 14, 251-266.

Endler, N. S., \& Parker, J. D. A. (1990). Multidimensional assessment of coping: A critical evaluation. Journal of Personality and Social Psychology, 58, 844-854.

Fiebert, M. S., \& Gonzalez, D. M. (1997). College women who initiate assaults on their male partners and the reasons offered for such behavior. Psychological Report, $80,583-90$.

Folkman, S., \& Lazarus, R. S. (1980). An analysis of coping in a middle-aged community sample. Jourrial of Health and Social Behavior, 21, 219-239.

Folkman, S., \& Lazarus, R. S. (1985). If it changes it must be a process: Study of emotion and coping during three stages of a college examination. Journal of Personality and Social Psychology, 48, 150-170.

Folkman, S., \& Moskowitz, J. T. (2004). Coping: Pitfalls and promise. Annual Review of Psychology, 55, 745-774.

Follingstad, D. R., Rutledge, L. L., Berg, B. J., Hause, E. S., \& Polek, D. S. (1990). The role of emotional abuse in physically abusive relationships. Journal of Family Violence, 5, 107-120. 
Gail, T. L., \& Evans, D. R. (2001). The dimensionality of cognitive appraisal and its relationship to physical and psychological well-being. The Journal of Psychology, $121,539-546$.

Garson, G. D. Reliability analysis. In Statnotes : Topics in multivariate analysis. Retrieved April 10, 2007 from www2.chass.ncsu.edu/garson/pa765/statnote.htm.

Garson, G. D. Testing of assumptions. In Statnotes : Topics in multivariate analysis. Retrieved April 6, 2007 from www2.chass.ncsu.edu/garson/pa765/statnote.htm.

Gaudreau, P., Blondin, J.- P., \& Lapierre, A.- M. (2002). Atheletes' coping during a competition: Relationship of coping strategies with positive affect, negative affect, and performance-goal discrepancy. Psychology of Sport and Exercise, 3, $125-150$.

Gibson, L. E., Leitenberg, H. (2001). The impact of child sexual abuse and stigma on methods of coping with sexual assault among undergraduate women. Child Abuse and Neglect, 25, 1343-1361.

Golding, J. M. (1999). Intimate partner violence as a risk factor for mental disorders: A meta-analysis. Journal of Family Violence, 14, 99-132.

Goodman, L. A., Dutton, M. A., Weinfurt, K., \& Cook, S. (2003). The Intimate Partner Violence Strategies Index. Violence Against Women, 9, 163-186.

Goodman, L. A., Koss, M. P., Russo, N. F. (1993). Violence against women: Physical and mental health effects: ПI. Research findings. Applied and Preventive Psychology, 2, 79-89. 
Griffin, M. G., Resick, P. A., \& Yehuda, R. (2005). Enhanced cortisol suppression following dexamethasone administration in domestic violence survivors. American Journal of Psychiatry, 162, 1192-1199.

Hamby, S. L. (2005). Measuring Gender Differences in Partner Violence: Implications from Research on Other Forms of Violent and Socially Undesirable Behavior. Sex Roles, 52, 725-742.

Harned, M. S. (2001). Abused women or abused men? An examination of the context and outcomes of dating violence. Violence and Victims, 16, 269-285.

Heim, C., Owens, M. J., Plotsky, P. M., Nemeroff, C. B. (1997). Persistent changes in corticotropin-releasing factor systems due to early life stress: Relationship to the pathophysiology of major depression and post-traumatic stress disorder. Psychopharmacology Bulletin, 33, 185-192.

Heim, C., \& Nemeroff, C. B. (2001). The role of childhood trauma in the neurobiology of mood and anxiety disorders: Preclinical and clinical studies. Biological Psychiatry, 49, 1023-1039.

Heim, C., \& Nemeroff, C. B. (2002). Neurobiology of early life stress: Clinical studies. Seminars in Clinical Neuropsychiatry, 7, 147-159.

Heim, C., Newport, D. J., Bonsall, R., Miller, A. H., \& Nemeroff, C. B. (2001). Altered pituitary-adrenal axis responses to provocative challenge tests in adult survivors of childhood abuse. American Journal of Psychiatry, 158, 575-581.

Heim, C., Newport, D. J., Heit, S., Graham, Y. P., Wilcox, M., Bonsall, R., Miller, A. H., \& Nemeroff, C. B. (2000). Pituitary-adrenal and autonomic responses to stress in 
women after sexual and physical abuse in childhood. Journal of the American Medical Association, 284, 592-597.

Heim, C., Newport, D. J., Wagner, D., Wilcox, M. M., Miller, A. H., \& Nemeroff, C. B. (2002). The role of early adverse experience and adulthood stress in the prediction of neuroendocrine stress reactivity in women: A multiple regression analysis. Depression and Anxiety, 15, 117-125.

Helgeson, V. S. (2003). Social support and quality of life. Quality of Life Research, 12, 25-31.

Herbert, T. B., Silver, R. C., \& Ellard, J. H. (1991). Coping with an abusive relationship: I. How and why do women stay? Journal of Marriage and the Family, 53, 311325.

Heszen-Niejodek, I. (1997). Coping style and its role in coping with stressful encounters. European Psychologist, 2, 342-351.

Hoffman, P. (1984). Psychological abuse of women by spouses and live-in lovers. Women and Therapy, 3, 37-47.

Houskamp, B. M., \& Foy, D. W. (1991). The assessment of posttraumatic stress disorder in battered women. Journal of Interpersonal Violence, 6, 367-375.

Howard, D. E., \& Wang, M. Q. (2003). Risk profiles of adolescent girls who were victims of dating violence. Adolescence, 38, 1-14.

Inslicht, S. S., Marmar, C. R., Neylan, T. C., Metzler, T. J., Hart, S. L., Otte, C., McCaslin, S. E., Larkin, G. L., Hyman, K. B., Baum, A. (2006). Increased cortisol in women with intimate partner violence-related posttraumatic stress disorder. Psychoneuroendocrinology, 31, 825-838. 
Johnson, J. G., Alloy, L. B., Panzarella, C., Metalsky, G. I., Rabkin, J. G., Williams, J. B., \& Abramson, L. Y. (2001). Hopelessness as a mediator of the association between social support and depressive symptoms: Findings of a study of men with HIV. Journal of Consulting and Clinical Psychology, 69, 1056-1060.

Johnson, S. B., Frattaroli, S., Campbell, J., Wright, J., Pearson-Fields, A. S., \& Cheng, T. L. (2005). "I know what love means." Gender-based violence in the lives of adolescents. Journal of Women's Health, 14, 172-179.

Judd, L. L., Akiskal, H. S., \& Paulus, M. P. (1997). The role and clinical significance of subsyndromal depressive symptoms (SSD) in unipolar major depressive disorder. Journal of Affective Disorders, 45, 5-17.

Judd, C. M., \& Kenny, D. A. (1981). Process analysis: Estimating mediation in treatment evaluations. Evaluation Review, 5, 602-619.

Kaniasty, K. Z., \& Norris, F. H. (1993). A test of the social support deterioration modeI in the context of natural disaster. Journal of Personality and Social Psychology, $64,395-408$.

Katz, J., \& Arias, I. (1999). Psychological abuse and depressive symptoms in dating women: Do different types of abuse have differential effects? Journal of Family Violence, 14, 281-295.

Keller-Wood, M. E., \& Dallman, M. F. (1984). Corticosteroid inhibition of ACTH secretion. Endocrinology Reviews, 5, 1-23.

Kemp, A., Green, B. L., Hovanitz, C., \& Rawlings, E. I. (1995). Incidence and correlates of posttraumatic stress disorder in battered women. Journal of Interpersonal Violence, 10, 43-55. 
Kenny, D. A., Kashy, D. A., \& Bolger, N. (1998). Data analysis in social psychology. In D. T. Gilbert, S. T. Fiske, \& G. Lindzey (Eds.), The handbook of social psychology ( $4^{\text {th }}$ ed., pp. 233-265). New York: Oxford University Press.

King, D. W., King, L. A., Foy, D. W., \& Gudanowski, D. M. (1996). Prewar factors in combat-related posttraumatic stress disorder: Structural equation modeling with a national sample of female and male Vietnam veterans. Journal of Consulting and Clinical Psychology, 64, 520-531.

King, D. W., King, L. A., Gudanowski, D. M., \& Vreven, D. L. (1995). Alternative representations of war zone stressors: Relationships to posttraumatic stress disorder in male and female Vietnam veterans. Journal of Abnormal Psychology, 104, 184-196.

Kirschbaum, C., Kudielka, B. M., Gaab, J., Schommer, N. C., \& Hellhammer, D. H. (1999). Impact of gender, menstrual cycle phase, and oral contraceptives on the activity of the hypothalamus-pituitary-adrenal axis. Psychosomatic Medicine, 61, $154-162$.

Knutsson, U., Stierna, P., Marcus, C., Carlstedt-Duke, J., Carlstrom, K., \& Bronnegard, M. (1995). Effects of intranasal glucocorticoids on endogenous glucocorticoid peripheral and central function. Journal of Endocrinology, 144, 301-310.

Kocot, T., \& Goodman, L. (2003). The roles of coping and social support in battered women's mental health. Violence Against Women, 9, 323-346.

Korte, S. M., Bouws, G. A., \& Bohus, B. (1993). Central actions of corticotropinreleasing hormone (CRH) on behavioral, neuroendocrine, and cardiovascular 
regulation: Brain corticoid receptor involvement. Hormones and Behavior, 27, 167-183.

Kouzis, A. C., \& Eaton, W. W. (1998). Absence of social networks, social support and health services utilization. Psychological Medicine, 28, 1301-1310.

Kubany, E. S., Hanes, S. N., Leisen, M. B., Owens, J. A., Kaplan, A. S., Watson, S. B., \& Burns, K. (2000). Development and preliminary validation of a brief broadspectrum measure of trauma exposure: The traumatic life events questionnaire. Psychological Assessment, 12, 210-224.

Kuffel, S., \& Katz, J. (2002). Preventing physical, psychological, and sexual aggression in college dating relationships. Journal of Primary Prevention, 22, 361-374.

Lazarus, R. S. \& Folkman, S. (1984). Stress, appraisal, and coping. New York: Springer.

Lazarus, R. S., \& Launier, R. (1978). Stress-related transactions between person and environment. In L. A. Pervin \& M. Lewis (Eds.), Perspectives in interactional psychology. New York: Plenum.

Lemieux A. M., \& Coe C. L. (1995). Abuse-related posttraumatic stress disorder: Evidence for chronic neuroendocrine activation in women. Psychosomatic Medicine, 57, 105-115.

Lerner, C. F., \& Kennedy, L. T. (2000). Stay-leave decision making in battered women: Trauma, coping, and self-efficacy. Cognitive Therapy and Research, 24, 215-232.

Levendosky, A. A., Bogat, G. A., Theran, S. A., Trotter, J. S., von Eye, A., \& Davidson, W. S. II (2004). The social networks of women experiencing domestic violence. American Journal of Community Psychology, 34, 95-109. 
Lupien, S. J., Nair, N. P. V., Briere, S., Maheu, F., Tu, M. T., Lemay, M., McEwen, B. S., \& Meany, M. (1999). Increased cortisol levels and impaired cognition in human aging: Implications for depression and dementia in later life. Reviews in Neuroscience, 10, 117-139.

MacKinnon, D. P., Lockwood, C. L., Hoffman, J. M., West, S. G., \& Sheets, V. (2002). A comparison of methods to test mediation and other intervening variable effects. Psychological Methods, 7, 83-104.

Makepeace, J. (1981). Courtship violence among college students. Family Relations, 30, 97-102.

Makepeace, J. (1986). Gender differences in courtship violence victimization. Family Relations, 35, 383-388.

Marais, A., de Villiers, P. J. T., Moller, A. T., \& Stein, D. J. (1999). Domestic violence in patients visiting general practitioners - Prevalence, phenomenology, and association with psychopathology. South African Journal of Medicine, 89, 635640.

Matheson, K. (June, 2003). Responding to dating abuse. Paper presented at the Annual Convention of the Canadian Psychological Association, Hamilton, ON.

Matheson, K., \& Anisman, H. (2003). Systems of coping associated with dysphoria, anxiety and depressive illness: A multivariate profile perspective. Stress, 6, 223234.

Matheson, K., Kelly, O., Cole, B., Tannenbaum, B., Dodd, C., \& Anisman, H. (2005). Parental bonding and depressive affect: The mediating role of coping resources. British Journal of Social Psychology, 44, 371-395. 
Matheson, K., Skomorovsky, A., Fiocco, A., \& Anisman, H. (2007). The limits of 'adaptive' coping : Well-being and mood reactions to stressors among women in abusive dating relationships. Stress, 10, 75-91.

McCrae, R. R. (1984). Situational determinants of coping responses: Loss, threat, and challenge. Journal of Personality and Social Psychology, 46, 919-928.

McEwen, B.S. (2000). The neurobiology of stress: From serendipity to clinical relevance. Brain Research, 886, 172-189.

Mellman, T. A., Randolph, C. A., Brawman-Mintzer, O., Flores, L. P., \& Milanes, F. J. (1992). Phenomenology and course of psychiatric disorders associated with combat-related posttraumatic stress disorder. American Journal of Psychiatry, $149,1568-1574$.

Merali, Z., Du, L., Hrdina, P., Palkovits, M., Faludi, G., Poulter, M. O., \& Anisman, H. (2004). Dysregulation in the suicide brain: mRNA expression of corticotropin releasing hormone receptors and GABAA receptor subunits in frontal cortical brain region. Journal of Neuroscience, 24, 1478-1485.

Miller, D. T., \& Porter, C. A. (1983). Self-blame in victims of violence. Journal of Social Issues, 39, 139-152.

Miller, S. M. (1992). Individual differences in the coping process: What to know and when to know it. In B. N. Carpenter (Ed.), Personal coping: Theory, research, and application (pp.77-91). Westport, CT: Praeger Publishers/Greenwood Publishing Group, Inc. 
Mitchell, R. E., \& Hodson, C. A. (1983). Coping with domestic violence: Social support and psychological health among battered women. American Journal of Community Psychology, 11, 629-654.

Mitchell, R. E., \& Hodson, C. A. (1986). Coping and social support among battered women: An ecological perspective. In S. E. Hobfoll (Ed.), Stress, social support and women. The series in clinical and community psychology (pp. 153-169). Hemisphere, New York.

Miyazaki, T., Ishikawa, T., Nakata, A., Sakurai, T., Miki, A., Fujita, O., Kobayashi, F., Haratani, T., Iimori, H., Sakami, S., Fujioka, Y., \& Kawamura, N. (2005). Association between perceived social support and Th1 dominance. Biological Psychology, 70, 30-37.

Momose, K., Inui, A., Asakawa, A., Ueno, N., Nakajima, M., Fujimiya, M., \& Kasuga, M. (1999). Intracerebroventricularly administered corticotropin-releasing factor inhibits food intake and produces anxiety-like behaviour at very low doses in mice. Diabetes Obesity and Metabolism, 1, 281-284.

Nemeroff, C. B., Bissette, G., Akil, H., \& Fink, M. (1991). Neuropeptide concentrations in the cerebrospinal fluid of depressed patients treated with electroconvulsive therapy. Corticotrophin-releasing factor, beta-endorphin and somatostatin. British Journal of Psychiatry, 158, 59-63.

Nemeroff, C. B., Widerlov, E., Bissette, G., Walleus, H., Karlsson, I., Eklund, K., Kilts, C. D., Loosen, P. T., \& Vale, W. (1984). Elevated concentrations of CSF corticotropin-releasing factor-like immunoreactivity in depressed patients. Science, 226, 1342-1344. 
Nixon, R. D. V., Resick, P. A., \& Nishith, P. (2004). An exploration of comorbid depression among female victims of intimate partner violence with posttraumatic stress disorder. Journal of Affective Disorders, 82, 315-320.

Nolen-Hoeksema, S. (2000). The role of rumination in depressive disorders and mixed anxiety/depressive symptoms. Journal of Abnormal Psychology, 109, 504-511.

Nunally, J. (1978). Psychometric theory ( ${ }^{\text {nd }}$ Edition). New York: McGraw Hill.

O'Leary, K. D. (1999). Developmental and affective issues in assessing and treating partner aggression. Clinical Psychology: Science and Practice, 6, 400-414.

O'Leary, K. D. (2001). Psychological abuse: A variable deserving critical attention in domestic violence. In K. D. O’Leary \& R. D. Maiuro (Eds.), Psychological abuse in violent domestic relations. New York: Springer Publishing Company.

Olson, L. N., Fine, M. A., \& Lloyd, S. A. (2005). Theorizing about aggression between intimates. In V. L. Bengston, A. C. Acock, K. R. Allen, P. Dilworth-Anderson, \& D. M. Klein (Eds.), Sourcebook of family theory and research. Sage Publications: London.

Orava, T. A., McLeod, P. J., \& Sharpe, D. (1996). Perceptions of control, depressive symptomatology, and self-esteem of women in transition from abusive relationships. Journal of Family Violence, 11, 167-188.

Pagelow, M. D. (1984). Family violence. New York: Praeger.

Pape, K. \& Arias, I. (2000). The role of perceptions and attributions in battered women's intentions to permanently end their violent relationships. Cognitive Therapy and Research, 24, 201-214. 
Peacock, E. J., \& Wong, P. T. P. (1990). The stress appraisal measure (SAM): A multidimensional approach to cognitive appraisal. Stress Medicine, 6, 227-236.

Penza, K. M., Heim, C., \& Nemeroff, C. B. (2003). Neurobiological effects of childhood abuse: Implications for the pathophysiology of depression and anxiety. Archives of Women's Mental Health, 6, 15-22.

Pico-Alfonso, M. A. (2005). Psychological intimate partner violence: The major predictor of posttraumatic stress disorder in abused women. Neuroscience and Biobehavioral Reviews, 29, 181-193.

Pico-Alfonso, M. A., Garcia-Linares, M. I., Celda-Navarro, N., Herbert, J., \& Martinez, M. (2004). Changes in cortisol and dehydroepiandrosterone in women victims of physical and psychological intimate partner violence. Biological Psychiatry, 56, $233-40$.

Pipes, R. B., \& LeBov-Keeler, K. (1997). Psychological abuse among college women in exclusive heterosexual dating relationships. Sex Roles, 36, 585-603.

Preacher, K. J., \& Hayes, A. F. (in review). Asymptotic and resampling strategies for assessing and comparing indirect effects in simple and multiple mediator models. Manuscript submitted for publication.

Preacher, K. J., \& Hayes, A. F. (2004). SPSS and SAS procedures for estimating indirect effects in simple mediation models. Behavior Research Methods, Instruments, \& Computers, 36, 717-731.

Pruessner, J. C., Wolf, O. T., Hellhammer, D. H., Buske-Kirschbaum, A., von Auer, K., Jobst, S., Kaspers, F., \& Kirschbaum, C. (1997). Free cortisol levels after 
awakening: A reliable biological marker for the assessment of adrenocortical activity. Life Science, 61, 2539-2549.

Rasmusson, A. M., Vythilingam, A., Morgan, C. A. III (2003). The neuroendocrinology of posttraumatic stress disorder: New directions. CNS Spectrum, 8, 651-667.

Ravindran A.V., Matheson, K., Griffiths J, Merali Z., \& Anisman H. (2002). Stress, coping, uplifts, and quality of life in subtypes of depression. Journal of Affective Disorders, 71, 121-131.

Reinberg, A. E., Touitou, Y., Soudant, E., Bernard, D., Bazin, R., \& Mechkouri, M. (1996). Oral contraceptives alter circadian rhythm parameters of cortisol, melatonin, blood pressure, heart rate, skin blood flow, transepidermal water loss, and skin amino acids of healthy young women. Chronobiology International, 13, 199-211.

Resnick, H. S., Kilpatrick, D. G., Best, C. L., \& Kramer, T. L. (1992). Vulnerabilitystress factors in development of posttraumatic stress disorder. Journal of Nervous and Mental Disorders, 180, 424-430.

Rickert, V. I., Wiemann, C. M., Vaughan, R. D., \& White, J. W. (2004). Rates and risk factors for sexual violence among an ethnically diverse sample of adolescents. Archives of Pediatric and Adolescent Medicine, 158, 1132-1139.

Roemer, L., \& Borkovec, T. D. (1994). Effects of suppressing thoughts about emotional material. Journal of Abnormal Psychology, 103, 467-474.

Rosen, K. H. (1996). The ties that bind women to violent premarital relationships: Processes of seduction and entrapment. In D. D. Cahn \& S. A. Lloyd (Eds.), 
Family violence from a communication perspective (pp. 151-176). Thousand Oaks, CA: Sage Publications.

Saltzman, L. E., Green, Y. T., Marks, J. S., \& Thacker, S. B. (2000). Violence against women as a public health issue. Comments from the CDC. American Journal of Preventive Medicine, 19, 325-329.

Sanchez, M. M., Young, L. J., Plotsky, P. M., \& Insel, T. R. (1999). Autoradiographic and in situ hybridization localization of corticotropin-releasing factor 1 and 2 receptors in nonhuman primate brain. Journal of Comparative Neurology, 408, 365-377.

Sapolsky, R. M., Romero, L. M., \& Munck, A. U. (2000). How do glucocorticoids influence the stress response? Integrating permissive, suppressive, stimulatory, and preparative actions. Endocrinology Reviews, 21, 55-89.

Saunders, D. G. (1986). When battered women use violence: Husband-abuse or selfdefense? Violence and Victims, 1, 47-60.

Sauvage, M., \& Steckler, T. (2001). Detection of corticotropin-releasing hormone receptor 1 immunoreactivity in cholinergic, dopaminergic and noradrenergic neurons of the murine basal forebrain and brainstem nuclei--potential implication for arousal and attention. Neuroscience, 104, 643-652.

Seedat, S., Stein, M. B., Kennedy, C. M., Hauger, R. L. (2003). Plasma cortisol and neuropeptide $\mathrm{Y}$ in female victims of intimate partner violence. Psychoneuroendocrinology 28, 796-808. 
Shrout, P. E., \& Bolger, N. (2002). Mediation in experimental and nonexperimental studies: New procedures and recommendations. Psychological Methods, 7, 422445 .

Slashinski, M. J., Coker, A. L., \& Davis, K. E. (2003). Physical aggression, forced sex, and stalking victimization by a dating partner: An analysis of the National Violence Against Women Survey. Violence and Victims, 18, 595-617.

Smith, J. A., Lumley, M. A., \& Longo, D. J. (2002). Contrasting emotional approach coping with passive coping for chronic myofascial pain. Annals of Behavioral Medicine, 24, 326-335.

Smith, P. H., Thornton, G. E., DeVellis, R., Earp, J., \& Coker, A. (2002). A populationbased study of the prevalence and distinctiveness of battering, physical assault, and sexual assault in intimate relationships. Violence Against Women, 8, 12081232.

Sobel, M. E. (1982). Asymptotic intervals for indirect effects in structural equations models. In S. Leinhart (Ed.), Sociological methodology 1982 (pp. 290-312). San Francisco, CA: Jossey-Bass.

Stanton, A. L., Danoff-Burg, S., Cameron, C. L., \& Ellis, A. P. (1994). Coping through emotional approach: Problems of conceptualization and confounding. Journal of Personality and Social Psychology, 66, 350-362.

Stanton, A. L., Danoff-Burg, S., Cameron, C. L., Bishop, M., Collins, C. A., Kirk, S. B., \& Sworowski, L. A., \& Twillman, R. (2000a). Emotionally expressive coping predicts psychological and physical adjustment to breast cancer. Journal of Consulting and Clinical Psychology, 68, 875-882. 
Stanton, A. L., Kirk, S. B., Cameron, C. L., \& Danoff-Burg, S. (2000b). Coping through emotional approach: Scale construction and validation. Journal of Personality and Social Psychology, 78, 1150-1169.

Statistics Canada (2000). Family violence in Canada: A statistical profile. Canadian Centre for Justice Statistics.

Statistics Canada (2005). Family violence in Canada: A statistical profile. Canadian Centre for Justice Statistics.

Stein, M. B., Yehuda, R., Koverola, C., \& Hanna, C. (1997). Enhanced dexamethasone suppression of plasma cortisol in adult women traumatized by childhood sexual abuse. Biological Psychiatry, 42, 680-686.

Stein, M. B., \& Kennedy, C. (2001). Major depressive and post-traumatic stress disorder comorbidity in female victims of intimate partner violence. Journal of Affective Disorders, 66, 133-138.

Stokes, P. E. (1995). The potential role of excessive cortisol induced by HPA hyperfunction in the pathogenesis of depression. European Neuropsychopharmacology, 5, 77-82.

Straight, E. S., Harper, F. W. K., \& Arias, I. (2003). The impact of partner psychological abuse on health behaviors and health status in college women. Journal of Interpersonal Violence, 18, 1035-1054.

Straus, M.A. (1979). Measuring intrafamily conflict and violence: The Conflict Tactics (CT) scales. Journal of Marriage and the Family, 41, 75-88. 
Straus, M. A., Hamby, S. L., Boney-McCoy, S., \& Sugarman, D. B. (1996). The revised Conflict Tactics Scales (CTS2). Development and preliminary psychometric data. Journal of Family Issues, 17, 283-316.

Street, A. E., \& Arias, I. (2001). Psychological abuse and posttraumatic stress disorder in battered women: Examining the roles of shame and guilt. Violence and Victims, $16,65-78$.

Suls, J., \& Fletcher, B. (1985). The relative efficacy of avoidant and nonavoidant coping strategies: A meta-analysis. Health Psychology, 4, 249-288.

Taft, C. T., Murphy, C. M., King, L. A., Dedeyn, J. M., \& Musser, P. H. (2005). Posttraumatic stress disorder symptomatology among partners of men in treatment for relationship abuse. Journal of Abnormal Psychology, 114, 259-268.

Thoits, P. A. (1986). Social support as coping assistance. Journal of Consulting and Clinical Psychology, 54, 416-423.

Valentiner, D. P., Foa, E. B., Riggs, D. S., \& Gershuny, B. S. (1996). Coping strategies and posttraumatic stress disorder in female victims of sexual and nonsexual assault. Journal of Abnormal Psychology, 105, 455-458.

Valentiner, D. P., Holahan, C. J., \& Moos, R. J. (1994). Social support, appraisals of event controllability, and coping: An integrative model. Journal of Personality and Social Psychology, 66, 1094-1102.

Veith, R. C., Lewis, N., Langohr, J. I., Murburg, M. M., Ashleigh, E. A., Castillo, S., Peskind, E. R., Pascualy, M., Bissette, G., Nemeroff, C. B., \& Raskind, M. A. (1993). Effect of desipramine on cerebrospinal fluid concentrations of corticotropin-releasing factor in human subjects. Psychiatry Research, 46, 1-8. 
Vest, J. R., Catlin, T. K., Chen, J. J., \& Brownson, R. C. (2002). Multistate analysis of factors associated with intimate partner violence. American Journal of Preventive Medicine, 22, 156-164.

Wagner, P. J., \& Mongan, P. F. (1998). Validating the concept of abuse. Women's perceptions of defining behaviors and the effects of emotional abuse on health indicators. Archives of Family Medicine, 7, 25-29.

Waldrop, A. E., \& Resick, P. A. (2004). Coping among adult female victims of domestic violence. Journal of Family Violence, 19, 291-302.

Walker, L. (1984). The battered woman syndrome. New York: Springer.

Weiss, D. S., \& Marmar, C. R. (1997). The Impact of Events Scale - Revised. In J. P. Wilson, \& T. M. Keane (Eds.), Assessing psychological trauma and PTSD: A handbook for practitioners (pp. 399-411). New York: Guilford Press.

Wegner, D. M., Schneider, D. J., Knutson, B., \& McMahon, S. R. (1991). Polluting the stream of consciousness: The effect of thought suppression on the mind's environment. Cognitive Therapy and Research, 15, 141-152.

Welberg, L. A. M., \& Seckl, J. R. (2001). Prenatal stress, glucocorticoids and the programming of the brain. Journal of Neuroendocrinology, 13, 113-128.

West, S. G., \& Aiken, L. S. (1997). Toward understanding individual effects in multicomponent prevention programs: Design and analysis strategies. In K. J. Bryant, M. Windle, \& S. G. West (Eds.), The science of prevention: Methodological advances from alcohol and substance abuse research (pp. 167209). Washington, DC: American Psychological Association. 
White, J. W., \& Koss, M. P. (1991). Courtship violence: Incidence in a national sample of higher education students. Violence and Victims, 6, 247-256.

Wood, M. (2005). Bootstrapped confidence intervals as an approach to statistical inference. Organizational Research Methods, 8, 454-470.

Woods, S. J., \& Isenberg, M. A. (2001). Adaptation as a mediator of intimate abuse and traumatic stressing battered women. Journal of Interpersonal Violence, 14, 479491.

Yehuda, R. (2002). Current status of cortisol findings in post-traumatic stress disorder. Psychiatric Clinics of North America, 25, 341-368.

Yehuda R., Boisoneau D., Mason J. W., Giller, E. L. (1993). Glucocorticoid receptor number and cortisol excretion in mood, anxiety, and psychotic disorders. Biological Psychiatry, 34, 18-25.

Yehuda, R., Giller, E. L., Levengood, R. A., Southwick, S. M., \& Siever, L. J. (1995). Hypothalamic-pituitary-adrenal dysfunction in posttraumatic stress disorder. In Friedman, M. J., Charney, D. S., \& Deutch, A. Y. (Eds.), Neurobiological and clinical consequences of stress. Philadelphia, PA: Lippincott-Raven.

Yehuda, R., Golier, J. A., \& Kaufman, S. (2005). Circadian rhythm of salivary cortisol in Holocaust survivors with and without PTSD. American Journal of Psychiatry, $162,998-1000$.

Yehuda, R., Lowy, M. T., Southwick, S. M., Shaffer, D., \& Giller, E. L., Jr. (1991). Lymphocyte glucocorticoid receptor number in posttraumatic stress disorder. American Journal of Psychiatry, 148, 499-504. 
Yehuda, R., Resnick, H. S., Schmeidler, J., Yang, R. K., \& Pitman, R. K. (1998). Predictors of cortisol and 3-methoxy-4-hydroxyphenylglycol responses in the acute aftermath of rape. Biological Psychiatry, 43, 855-859.

Yehuda, R., Teicher, M. H., Trestman, R. L., Levengood, R. A., \& Siever, L. J. (1996). Cortisol regulation in posttraumatic stress disorder and depression: a chronobiological analysis. Biological Psychiatry, 40, 79-88.

Yoshihama, M. \& Horrocks, J. (2003). The relationship between intimate partner violence and PTSD: An application of Cox regression with time-varying covariates. Journal of Traumatic Stress, 16, 371-380.

Zakowski, S. G., Hall, M. H., Klein, L. C., \& Baum, A. (2001). Appraised control, coping, and stress in a community sample: A test of the goodness-of-fit hypothesis. Annals of Behavioral Medicine, 23, 158-165.

Zobel, A. W., Nickel, T., Kunzel, H. E., Ackl, N., Sonntag, A., Ising, M., \& Holsboer, F. (2000). Effects of the high-affinity corticotropin-releasing hormone receptor 1 antagonist R121919 in major depression: The first 20 patients treated. Journal of Psychiatric Research, 34, 171-181.

Zoellner, L. A., Foa, E. B., \& Brigidi, B. D. (1999). Interpersonal friction and PTSD in female victims of sexual and nonsexual assault. Journal of Trauma and Stress, 12, $689-700$. 
APPENDIX 1: Recruitment Materials 


\section{QUESTIONS FOR MASS TESTING (Study 1)}

Have you ever been in an intimate relationship which you, or someone close to you

(friend, family member), considered abusive (either emotionally, physically, or sexually)?

_ yes

n no

If yes, are you referring to your current relationship or a previous relationship that you were in?

current

previous 


\section{QUESTIONS FOR MASS TESTING (Study 2)}

1. Have you ever experienced abuse (either emotional, physical, or sexual)?

Yes
No

2. If yes, please indicate the type of abuse you experienced.

Emotional

Physical

Sexual

3. What relationship did you have to the perpetrator?

__ Romantic partner current partner or previous partner? (circle one)

Friend or acquaintance

Parent or caregiver

Other relative

Stranger

Other (please indicate):

4. At what age did this happen?

Before age 13

Between 13 and 17

After age 17 
APPENDIX 2: Informed Consent and Debriefing 


\section{Informed Consent (Study 1)}

The purpose of an informed consent is to ensure that you understand the purpose of the study and the nature of your involvement. The informed consent has to provide sufficient information such that you have the opportunity to determine whether you wish to participate in the study.

Study Title: Women's relationships, quality of life and reaction to stressors

Study Personnel: Kerry Sudom (Researcher, 520-2600 ext 2683)

Dr. Hymie Anisman (Faculty Investigator, 520-2699)

Dr. Kim Matheson (Faculty Investigator, 520-2648)

Renate Ewert Ysseldyk (Researcher, 520-2600 ext. 2683)

If you have any ethical concerns about how this study please contact Dr. M. Gick, Chair of Carleton University Ethics Committee for Psychological Research, 520-2600, ext. 2664 or Dr. J. Logan, Chair of Dept. of Psychology, 520-2600 ext. 2690.

Purpose and Task Requirements: The purpose of this study is to assess how your relationship with your present partner and/or your previous relationships impacts on your physical and psychological health and quality of life. In the first session, we are asking you to fill out a number of questionnaires regarding your personal characteristics (such as how you feel about yourself, what you're like), your psychological health and family history, as well as some potentially traumatic events you may have experienced (e.g. assault, war, sexual abuse, loss of a loved one) that may have affected how you deal with other events in your life. As well, we are asking you about detailed information about your relationship with your partner and other people. Further, we will be measuring several hormones that are present in your saliva, which may also give us clues about your stress levels and general health. In the second session, we will be asking you to view, as well as comment on a series of photographs with varied contents (including negative/graphic images). You will be given 2 experimental credits for participating in the two sessions.

Potential Risk and Discomfort: There are no physical risks in this study. There may be some discomfort when thinking about your relationships and the effects on you.

Anonymity/Confidentiality: The data collected in this study will be kept confidential. Your informed consent form, will be separated from your questionnaire and kept in a separate and secured file by one of the research investigators who will keep this information confidential. It will be associated with a code, and only this code will identify your questionnaire.

Right to Withdraw: Your participation in this study is entirely voluntary. At any point during the study you have the right to not complete certain questions or to withdraw with no penalty whatsoever.

I have read the above description of the study concerning how my intimate relationship may influence my well-being. The data collected will be used in research publications and/or for teaching purposes. My signature indicates that I agree to participate in the study, and this in no way constitutes a waiver of my rights.

Full Name (please print):

Participant Signature:

Date:

Researcher Signature:

Date: 


\section{Informed Consent (Study 2, part 1)}

The purpose of an informed consent is to ensure that you understand the purpose of the study and the nature of your involvement. The informed consent has to provide sufficient information such that you have the opportunity to determine whether you wish to participate in the study.

Study Title: Women's relationships, quality of life and reaction to stressors

Study Personnel: Kerry Sudom (Researcher, 520-2600 ext. 7513)

Dr. Hymie Anisman (Faculty Investigator, 520-2600 ext. 2699)

Dr. Kim Matheson (Faculty Investigator, 520-2600 ext. 2648)

Dr. Michael Wohl (Faculty Investigator, 520-2600 ext. 2908)

Carolynne Weir (Researcher, 520-2600 ext. 7513)

If you have any ethical concerns about how this study please contact Dr. C. Davis, Chair of Carleton University Ethics Committee for Psychological Research, 520-2600, ext. 2251 or Dr. M. Gick, Chair of Dept. of Psychology, 520-2600 ext. 2648.

Purpose and Task Requirements: The purpose of this study is to assess how your relationship with your present partner and/or your previous relationships impacts on your physical and psychological health and quality of life. We are asking you to fill out a number of questionnaires regarding your personal characteristics (such as how you feel about yourself, what you're like), your psychological health and family history, as well as some potentially traumatic events you may have experienced (e.g. assault, war, sexual abuse, loss of a loved one) that may have affected how you deal with other events in your life. As well, we are asking you about detailed information about your relationship with your partner and other people. Further, we will be measuring hormones that are present in your saliva, which may also give us clues about your stress levels and general health. We also will be asking you to view, as well as comment on photographs that may be potentially aversive, and your responses on this part of the study will be taped. You will be given 2 experimental credits for participating in the two sessions.

Potential Risk and Discomfort: There are no physical risks in this study. There may be some discomfort when thinking about your relationships and the effects on you.

Anonymity/Confidentiality: The data collected in this study will be kept confidential. Your informed consent form will be separated from your questionnaire and kept in a separate and secured file by one of the research investigators who will keep this information confidential. It will be associated with a code, and only this code will identify your questionnaire.

Right to Withdraw: Your participation in this study is entirely voluntary. At any point during the study you have the right to not complete certain questions or to withdraw with no penalty whatsoever.

I have read the above description of the study concerning how my intimate relationship may influence my well-being. The data collected will be used in research publications and/or for teaching purposes. My signature indicates that I agree to participate in the study, and this in no way constitutes a waiver of my rights.

Full Name (please print):

Participant Signature:

Date:

Researcher Signature:

Date: 


\section{Informed consent for videotaping (Study 2)}

We will be asking you to view, as well as comment on, a photograph depicting a potentially stressful scene. Specifically, we would like to ask you to speak for about two minutes about what you think is going on in the situation, and what were the circumstances leading up to the situation. Following this, you will be asked to speak for a minute about what you would do, and what your emotional responses would be, if you were in the situation depicted in the photograph. In order for us to look at the content of your responses, we would like to videotape you doing this task. The videotape will be kept in a secure place by the experimenters to ensure your privacy. Your signature below indicates that you have given consent to be videotaped for the purposes of this study:

Full Name (please print):

Participant Signature:

Date:

Researcher Signature:

Date: 


\section{Informed Consent (Study 2, Part 2)}

The purpose of an informed consent is to ensure that you understand the purpose of the study and the nature of your involvement. The informed consent has to provide sufficient information such that you have the opportunity to determine whether you wish to participate in the study.

Study Title: Women's relationships, quality of life and reaction to stressors

Study Personnel: Kerry Sudom (Researcher, 520-2600 ext. 7513)

Dr. Hymie Anisman (Faculty Investigator, 520-2600 ext. 2699)

Dr. Kim Matheson (Faculty Investigator, 520-2600 ext. 2648)

Dr. Michael Wohl (Faculty Investigator, 520-2600 ext. 2908)

Carolynne Weir (Researcher, 520-2600 ext. 7513)

If you have any ethical concerns about how this study please contact Dr. C. Davis, Chair of Carleton University Ethics Committee for Psychological Research, 520-2600, ext. 2251 or Dr. M. Gick, Chair of Dept. of Psychology, 520-2600 ext. 2648.

Purpose and Task Requirements: The purpose of this study is to assess experiences of stress and conflict in your relationship with your present partner and/or your previous relationships impact upon your physical and psychological health and quality of life. We are asking you to fill out a number of questionnaires regarding your personal characteristics (such as how you feel about yourself, what you're like), your psychological health and family history, as well as detailed information about your relationship with your partner and other people. Further, we will be measuring hormones that are present in your saliva, which may also give us clues about your stress levels and general health. You will be given 1 experimental credit for participating in this study.

Potential Risk and Discomfort: There are no physical risks in this study. There may be some emotional pain or discomfort when thinking about negative aspects of your past and present relationships, as well as any personal traumas that you have encountered in the past, and their effects on you. As well, viewing potentially stressful images may cause some distress or discomfort.

Anonymity/Confidentiality: The data collected in this study will be kept confidential. Your informed consent form will be separated from your questionnaire and kept in a separate and secured file by one of the research investigators who will keep this information confidential. It will be associated with a code, and only this code will identify your questionnaire.

Right to Withdraw: Your participation in this study is entirely voluntary. At any point during the study you have the right to not complete certain questions or to withdraw with no penalty whatsoever.

I have read the above description of the study concerning how my intimate relationship may influence my well-being. The data collected will be used in research publications and/or for teaching purposes. My signature indicates that I agree to participate in the study, and this in no way constitutes a waiver of my rights.

Full Name (please print):

Participant Signature:

Date:

Researcher Signature:

Date: 


\section{Debriefing (Study 1)}

In this study, we have been interested in assessing the quality of women's relationships, and the effects of this on women's mental health, quality of life and academic functioning. While most women are in relationships that are not abusive, unfortunately abuse is all too common in intimate relationships. Male violence against women, or women battering is one of the greatest causes of injury, mental disorders and death among women (Goodman et al., 1993). The recent Commonwealth Fund survey demonstrated that more than 4 million women are abused by their male partners every year, with half of them suffering from serious injuries and 2,000-4,000 dying as a direct result of the abuse (Plichta, 1996). Battered women live in constant threat of repeated and sometimes severe violence. Not surprisingly, a high incidence of mental health problems exists among women experiencing abuse, including depression, anxiety disorders, cognitive dissociations, feelings of helplessness, fatigue, listlessness, self-imposed isolation, alcohol and drug abuse, disruption of interpersonal relationships, somatic complaints and suicide attempts (Astin \& Foy, 1995; Astin \& Lawrence, 1993; Browne, 1993; Weaver \& Clum, 1995). There is evidence to suggest that this abuse is evident even at the point of women's early dating relationships. Indeed, young women are regarded as being at greater risk for experiencing such violence than older women. About $5 \%$ of women under the age of 25 reported at least one incident of violence in their current relationship within the past 12 months (compared to $1 \%$ of women 45 and older) (Statistics Canada, 1999).

While numerous researchers have examined the prevalence of psychological problems among battered women, these estimates vary substantially from study to study (Golding, 1999). In part the problem stems from the fact that much of this research is conducted following prolonged episodes of severe abuse among women in shelters or who are seeking counselling due to their abuse. Therefore it is hard to identify where the problem started (e.g., are there early life experiences that make some women more vulnerable to abusive relationships), and what factors help women to deal with an abusive relationship in terms of maintaining their own psychological and physical health and well-being.

Therefore, this study examines the dating relationships of women to ascertain the frequency of abuse in their relationships, the nature of the abuse encountered, and the ramifications of this abuse in terms of women's functional (e.g., academic performance), psychological (e.g., developing depression or stress disorders) and physical well-being (e.g., physical health and symptoms). We also want to see how much of women's past experiences, coping styles and social support may help them to deal with conflict and/or abuse their relationships. While many who have participated in this study may not be in abusive relationships, some are. We will be examining the differences among women as a function of the nature of their intimate relationships, and the effects such relationships have on women over the course of the year. If you believe you may be in an abusive relationship, we have attached some numbers you may contact to get more information and/or help. 
Contacts

The following people are involved in this research project and may be contacted at any time if you have any further questions about the project, what it means, or concerns about how it was conducted:

Dr. H. Anisman, Faculty Member, Department of Psychology, 520-2699

Dr. K. Matheson, Faculty Member, Dept. of Psychology, 520-2648

Kerry Sudom, Graduate Researcher, Department of Psychology, 520-2600 ext. 2683

Renate Ewert Ysseldyk, Graduate Researcher, Department of Psychology, 520-2600 ext. 2683

If you have any ethical concerns about how this study was conducted, please contact either of the following:

Dr. M. Gick, Chair of Carleton University Ethics Committee for Psychological Research, 520-2600, ext. 2664

Dr. J. Logan, Chair of Dept. of Psychology, 520-2600 ext. 2690

If you have any worries or concerns about your personal well-being, you can contact the following services:

\section{Carleton University Health and Counseling Services 520-6674}

Ottawa Rape Crisis Centre. Crisis line: counselors available for anonymous crisis calls: 5622333, 24 hours/day.

Sexual Assault Support Centre. Call $725-2160$ to find out about support groups, and/or accompaniments to police, court, or hospital. Or 24 hour support line: 234-2266.

Community Resource Centre. Have you been psychologically or physically abused? For support and information, call 591-3686.

If you would like more information about women and their intimate relationships, you can find valuable information at:

Womyn's Centre on Campus at 308 University Centre 520-2712. 


\section{Debriefing (Study 2, Part 1)}

In this study, we have been interested in assessing the quality of women's relationships, and the effects of this on women's mental health, quality of life and academic functioning. While most women are in relationships that are not abusive, unfortunately abuse is all too common in intimate relationships. Male violence against women, or women battering is one of the greatest causes of injury, mental disorders and death among women (Goodman et al., 1993). Battered women live in constant threat of repeated and sometimes severe violence. Not surprisingly, a high incidence of mental health problems exists among women experiencing abuse, including depression, anxiety disorders, cognitive dissociations, feelings of helplessness, fatigue, listlessness, self-imposed isolation, alcohol and drug abuse, disruption of interpersonal relationships, somatic complaints and suicide attempts (Astin \& Foy, 1995; Astin \& Lawrence, 1993; Browne, 1993; Weaver \& Clum, 1995). There is evidence to suggest that this abuse is evident even at the point of women's early dating relationships. Indeed, young women are regarded as being at greater risk for experiencing such violence than older women. About $5 \%$ of women under the age of 25 reported at least one incident of violence in their current relationship within the past 12 months (compared to $1 \%$ of women 45 and older) (Statistics Canada, 1999). Thus, it is important to identify the presence of abuse, and to understand the factors associated with entering into and maintaining an abusive relationship, early in dating relationships. It is important to be able to recognize if you, or someone you know, is in an abusive relationship so that further escalation of violence can be prevented.

While numerous researchers have examined the prevalence of psychological problems among battered women, these estimates vary substantially from study to study (Golding, 1999). In part the problem stems from the fact that much of this research is conducted following prolonged episodes of severe abuse among women in shelters or who are seeking counselling due to their abuse. Therefore it is hard to identify where the problem started (e.g., are there early life experiences that make some women more vulnerable to abusive relationships), and what factors help women to deal with an abusive relationship in terms of maintaining their own psychological and physical health and well-being.

Therefore, this study examines the dating relationships of women to ascertain the frequency of abuse in their relationships, the nature of the abuse encountered, and the ramifications of this abuse in terms of women's functional (e.g., academic performance), psychological (e.g., developing depression or stress disorders) and physical well-being (e.g., physical health and symptoms). We also want to see how much of women's past experiences, coping styles and social support may help them to deal with conflict and/or abuse their relationships. While many who have participated in this study may not be in abusive relationships, some are. We will be examining the differences among women as a function of the nature of their intimate relationships, and the effects such relationships have on women over the course of the year. If you believe you may be in an abusive relationship, we have attached some numbers you may contact to get more information and/or help. 


\section{Debriefing (Study 2, Part 2)}

In this study, we have been interested in assessing the quality of women's relationships, and the effects of this on women's mental health, quality of life and academic functioning. In this study, we are interested in considering the consequences of imagining alterative outcomes of negative events for psychological well-being among women who may have experienced abuse within an intimate relationship. A lack of deeply held resentments has been described as an implicit component of positive mental health (Beck, 1992). Forgiveness is considered a process that releases resentment (Wohl et al., 2004). Thus, it follows that the accomplishment of forgiveness should be associated with mental health. Prior research on sexual assault victims (Branscombe, et al., 2003) has indicated that thinking about how one could have avoided the negative life event from occurring can result in poor psychological well-being. In the present study, therefore, we are interested in examining whether self-forgiveness in the context of relationship conflict buffers individuals from the negative effects of imagining alternative outcomes on psychological (e.g., depression, lowered self-esteem, perceived lack of control over life) and physical (e.g., physical health and symptoms) well-being. Identifying the role of forgiveness in recovery will provide important information for the development and implementation of intervention programs designed to assist women who have experienced an abuse within an intimate relationship. As well, in this study we are assessing the assignment of blame for relationship conflicts, since past research has shown that abuse victims often erroneously attribute blame for the event to themselves. It is important to recognize that at times, in situations of relationship conflict and abuse, individuals sometimes take responsibility for events that they should not. This in turn may have negative effects on well-being.

While many who have participated in this study may not be in abusive relationships, some are. We will be examining the differences among women as a function of the nature of their intimate relationships, and the effects such relationships have on women over the course of the year. It is important to identify the presence of abuse, and to understand the factors associated with entering into and maintaining an abusive relationship, early in dating relationships, and to assess the effects of forgiveness, assignment of blame, and counterfactual thoughts on well-being. It is important to be able to recognize if you, or someone you know, is in an abusive relationship so

that further escalation of violence can be prevented. As well, it is important to be aware that if you are in such a relationship, you are not to blame. If you believe you may be in an abusive relationship, we have attached some numbers you may contact to get more information and/or help. 
Contacts

The following people are involved in this research project and may be contacted at any time if you have any further questions about the project, what it means, or concerns about how it was conducted:

Dr. H. Anisman, Faculty Member, Department of Psychology, 520-2600 ext. 2699

Dr. K. Matheson, Faculty Member, Dept. of Psychology, 520-2600 ext. 2684

Dr. M. Wohl, Faculty Member, Department of Psychology, 520-2600 ext. 2908

Kerry Sudom, Graduate Researcher, Department of Psychology, 520-2600 ext. 7513

If you have any ethical concerns about how this study was conducted, please contact either of the following:

Dr. C. Davis, Chair of Carleton University Ethics Committee for Psychological Research, 520-2600, ext. 2251

Dr. M. Gick, Chair of Dept. of Psychology, 520-2600 ext. 2648

If you have any worries or concerns about your personal well-being, you can contact the following services:

Carleton University Health and Counseling Services 520-6674

Ottawa Rape Crisis Centre. Crisis line: counselors available for anonymous crisis calls: $562-$ 2333, 24 hours/day.

Sexual Assault Support Centre. Call $725-2160$ to find out about support groups, and/or accompaniments to police, court, or hospital. Or 24 hour support line: 234-2266.

Community Resource Centre. Have you been psychologically or physically abused? For support and information, call 591-3686.

If you would like more information about women and their intimate relationships, you can find valuable information at:

Womyn's Centre on Campus at 308 University Centre 520-2712. 
APPENDIX 3: Questionnaires 


\section{Background}

Age

$\operatorname{Sex}$

Ethnic/racial background

Religious affiliation

Current GPA

Year in program

The following questions are important for our analysis of stress hormones:

What time did you wake up this morning?

Are you currently being treated for any physical condition?

No___ Yes____ If yes, please specify

Are you on any of the following medications (please check all that apply):

Birth control pill

Anti-inflammatories (please specify)

Anti-depressives (please specify)

Anti-anxieties (please specify)

Other Prescription drugs (please specify

Have you ever been in therapy? (please check the one that best applies)

No, I have never been in therapy

- Yes, but I am no longer

Y Yes, and still am

If yes, how long ago were you in, or have you been in therapy?

Began ___ month/year and continued until ___ month/year 


\section{Relationship Status}

What is your relationship status? (please check the one that applies best to you)

Single, and not seeing anyone

Am going out with someone

Am living with an intimate other

Have recently broke up ........

Please specify how weeks ago you broke up

Please specify who initiated the break up:

me

my partner

mutual decision

If you are currently involved with someone,

How long have you been in this relationship?

years OR months

\section{If you are currently involved with someone,}

Does the person live in Ottawa?

No___ Yes____ If no, where does s/he live?

Does s/he attend Carleton University? No

Yes

In your current relationship, has this person ever been physically aggressive toward you?

0

Never

Only once
2

A few times
3

4

\section{Several times Frequently}

In your current relationship, has this person ever ridiculed, insulted or humiliated you without reason?

0

1

2

3

4

Never Only once A few times Several times Frequently

In your current relationship, have you ever felt afraid of this person?

0

1

2

3

4

Never Only once A few times Several times Frequently

If you think that you may be in an abusive relationship, how long would you estimate that it has been going on for?

weeks or months 


\section{CONFLICT TACTICS SCALE}

\section{Study 1 instructions:}

No matter how well a couple gets along, there are times when they disagree, get annoyed with the other person, want different things from each other, or just have fights because they are in a bad mood, tired, or for some other reason. Couples also have many different ways of trying to settle their differences. Below is a list of things that might have been done to you by your partner. For each statement, please indicate how often your partner has behaved this way toward you in the past month by writing a number in the space to the right of each statement using the following rating scale. If you are not currently in a relationship, please answer the questions in relation to your most recent relationship:

$\begin{array}{cccccc}0 & 1 & 2 & 3 & 4 & 5 \\ \text { Never } & \text { Once } & \text { Twice } & 3-5 & 6-10 & \text { More than } 10 \\ & & & \text { times } & \text { times } & \text { times }\end{array}$

\section{Study 2 instructions:}

No matter how well a couple gets along, there are times when they disagree, get annoyed with the other person, want different things from each other, or just have fights because they are in a bad mood, tired, or for some other reason. Couples also have many different ways of trying to settle their differences, and may have different perceptions of what constitutes effective means of dealing with such conflicts. For each statement below, please indicate to what extent you feel the behavior described is abusive (even if it has not happened to you) by writing a number in the space to the right of each statement using the following 5-point rating scale:

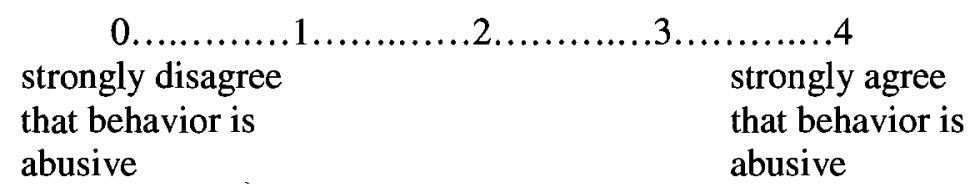

1. My partner showed care for me even though we disagreed.

2. My partner explained his side of a disagreement to me.

3. My partner insulted or swore at me.

4. My partner threw something at me that could hurt.

5. My partner twisted my arm or pulled my hair.

6. I had a sprain, bruise, or small cut because of a fight with my partner.

7. My partner showed respect for my feelings about an issue. 
8. My partner made me have sex without a condom.

9. My partner pushed or shoved me.

10. My partner used force (like hitting, holding down, or using a weapon) to make me have oral or anal sex.

11. My partner used a knife or gun on me.

12. I passed out from being hit on the head by my partner in a fight.

13. My partner called me fat or ugly.

14. My partner punched or hit me.

15. My partner destroyed something belonging to me.

16. I went to a doctor because of a fight with my partner.

17. My partner choked me.

18. My partner shouted or yelled at me.

19. My partner pushed me against a wall.

20. My partner was sure we could work things out.

21. I needed to see a doctor because of a fight with my partner, but I didn't.

22. My partner beat me up.

23. My partner grabbed me.

24. My partner used force (like hitting, holding down, or using a weapon) to make me have sex.

25. My partner stomped out of the room or house during the disagreement.

26. My partner insisted on sex when I didn't want to (but didn't use physical force).

27. My partner slapped me.

28. I had a broken bone from a fight with my partner.

29. My partner used threats to make me have oral or anal sex.

30. My partner suggested a compromise to a disagreement.

31. My partner burned or scalded me on purpose.

32. My partner insisted I have oral or anal sex (but didn't use physical force). 
33. My partner accused me of being a lousy lover.

34. My partner did something to spite me.

35. My partner threatened to hit or throw something at me.

36. I felt physical pain that still hurt the next day because of a fight with my partner.

37. My partner kicked me.

38. My partner used threats to make me have sex.

39. My partner agreed to try a solution to a disagreement I suggested. 


\section{CONFLICT TACTICS SCALE (Study 2)}

No matter how well a couple gets along, there are times when they disagree, get annoyed with the other person, want different things from each other, or just have fights because they are in a bad mood, tired, or for some other reason. Couples also have many different ways of trying to settle their differences. Below is a list of things that might have been done to you by your partner, or that you may have done. For each statement, please indicate how often your partner has behaved this way toward you in the past month by writing a number in the space to the right of each statement using the following rating scale. If you are not currently in a relationship, please answer the questions in relation to your most recent relationship:

$\begin{array}{cccccc}0 & 1 & 2 & 3 & 4 & 5 \\ \text { Never } & \text { Once } & \text { Twice } & 3-5 & 6-10 & \text { More than } 10 \\ & & & \text { times } & \text { times } & \text { times }\end{array}$

1. I showed my partner I cared even though we disagreed.

2. My partner showed care for me even though we disagreed.

3. I explained my side of a disagreement to my partner.

4. My partner explained his side of a disagreement to me.

5. I insulted or swore at my partner.

6. My partner insulted or swore at me.

7. I threw something at my partner that could hurt.

8. My partner threw something at me that could hurt.

9. I twisted my partner's arm or pulled his hair.

10. My partner twisted my arm or pulled my hair.

11. I had a sprain, bruise, or small cut because of a fight with my partner.

12. My partner had a sprain, bruise, or small cut because of a fight with me.

13. I showed respect for my partner's feelings about an issue.

14. My partner showed respect for my feelings about an issue.

15. I made my partner my partner have sex without a condom.

16. My partner made me have sex without a condom.

17. I pushed or shoved my partner.

18. My partner pushed or shoved me.

19. I used force (like hitting, holding down, or using a weapon) to make my partner have oral or anal sex. 


$\begin{array}{cccccc}0 & 1 & 2 & 3 & 4 & 5 \\ \text { Never } & \text { Once } & \text { Twice } & 3-5 & 6-10 & \text { More than } 10\end{array}$

20. My partner used force to make me have oral or anal sex.

21. I used a knife or gun on my partner.

22. My partner used a knife or gun on me.

23. I passed out from being hit on the head by my partner in a fight.

24. My partner passed out from being hit on the head in a fight with me.

25. I called my partner fat or ugly.

26. My partner called me fat or ugly.

27. I punched or hit my partner.

28. My partner punched or hit me.

29. I destroyed something belonging to my partner.

30. My partner destroyed something belonging to me.

31. I went to a doctor because of a fight with my partner.

32. My partner went to a doctor because of fight with me.

33. I choked my partner.

34. My partner choked me.

35. I shouted or yelled at my partner.

36. My partner shouted or yelled at me.

37. I pushed my partner against a wall.

38. My partner pushed me against a wall.

39. I said I was sure we could work things out.

40. My partner was sure we could work things out.

41. I needed to see a doctor because of a fight with my partner, but I didn't.

42. My partner needed to see a doctor because of a fight with me, but he didn't.

43. I beat up my partner.

44. My partner beat me up. 


$\begin{array}{cccccc}0 & 1 & 2 & 3 & 4 & 5 \\ \text { Never } & \text { Once } & \text { Twice } & 3-5 & 6-10 & \text { More than } 10 \\ & & & \text { times } & \text { times } & \text { times }\end{array}$

45. I grabbed my partner.

46. My partner grabbed me.

47. I used force (like hitting, holding down, or using a weapon) to make my partner have sex.

48. My partner used force to make me have sex.

49. I stomped out of the room or house during a disagreement.

50. My partner stomped out of the room or house during a disagreement.

51. I insisted on sex when my partner didn't want to (but didn't use physical force).

52. My partner insisted on sex when I didn't want to (but didn't use physical force).

53. I slapped my partner.

54. My partner slapped me.

55. I had a broken bone from a fight with my partner.

56. My partner had a broken bone from a fight with me.

57. I used threats to make my partner have oral or anal sex.

58. My partner used threats to make me have oral or anal sex.

59. I suggested a compromise to a disagreement.

60. My partner suggested a compromise to a disagreement.

61. I burned or scalded my partner on purpose.

62. My partner burned or scalded me on purpose.

63. I insisted my partner have oral or anal sex (but didn't use physical force).

64. My partner insisted I have oral or anal sex (but didn't use physical force).

65. I accused my partner of being a lousy lover.

66. My partner accused me of being a lousy lover.

67. I did something to spite my partner.

68. My partner did something to spite me. 


$\begin{array}{cccccc}0 & 1 & 2 & 3 & 4 & 5 \\ \text { Never } & \text { Once } & \text { Twice } & 3-5 & 6-10 & \text { More than } 10 \\ & & & \text { times } & \text { times } & \text { times }\end{array}$

69. I threatened to hit or throw something at my partner.

70. My partner threatened to hit or throw something at me.

71. I felt physical pain that still hurt the next day because of a fight with my partner.

72. My partner still felt physical pain the next day because of a fight with me.

73. I kicked my partner.

74. My partner kicked me.

75. I used threats to make my partner have sex.

76. My partner used threats to make me have sex.

77. I agreed to try a solution to a disagreement my partner suggested.

78. My partner agreed to try a solution I suggested. 


\section{IMPACT OF EVENT SCALE - Revised}

\section{Study 1 instructions:}

The following is a list of difficulties people sometimes have after stressful life events. Please read each item and then indicate how distressing each difficulty has been for you during the past seven days as a result of a recent or past stressful event.

Please briefly describe the event:

\section{Study 2 instructions:}

a) Please consider whether you have experienced any type of maltreatment by your partner (e.g., verbal attacks, humiliation, threats, physical assault, sexual coercion) in the past month. If you have not experienced maltreatment by your partner, please think of a conflict or disagreement you had with your partner in the past month that you found particularly stressful.

Please briefly describe your experience of maltreatment, or the disagreement:

b) The following is a list of difficulties people sometimes have after stressful life events. Please consider the event from the previous page (question \#25 from Life Events Questionnaire) that you indicated you found the most stressful. Please read each of the items below and indicate how distressing each difficulty has been for you during the past seven days as a result of this stressful event.

Please briefly describe the event: 
During the past seven days, how much have you been distressed or bothered with these difficulties?

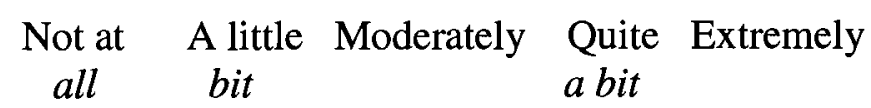

1. Any reminder brought back feelings about it. 0

$\begin{array}{llll}1 & 2 & 3 & 4\end{array}$

2. I had trouble staying asleep.

0

3. Other things kept making me think about it. 0

4. I felt irritable and angry.

5. I avoided letting myself get upset when I thought about it or was reminded of it.

6. I thought about it when I didn't mean to.

2

3

4

7. I felt as if it hadn't happened or wasn't real.

8. I stayed away from reminders about it.

0

9. Pictures about it popped into my mind.

0

10. I was jumpy and easily startled.

0

11. I tried not to think about it.

0

12. I was aware that I still had a lot of feelings 0 about it but I didn't deal with them.

13. My feelings about it were kind of numb.

14. I found myself acting or feeling like I was back at this time.

15. I had trouble falling asleep.

16. I had waves of strong feelings about it.

17. I tried to remove it from my memory.

1

2

3

4 
19. Reminders of it caused me to have physical 0 reactions, such as sweating, trouble breathing, nausea, or a pounding heart.

20. I had dreams about it.

21. I felt watchful and on guard.

22. I tried not to talk about it.
0

0

0
1

2

3

4

$\begin{array}{lllll}1 & 2 & 3 & 4\end{array}$

$\begin{array}{lllll}0 & 1 & 2 & 3 & 4\end{array}$




\section{COPING STRATEGIES SCALE}

We are interested in how you dealt with a specific disagreement between you and your partner. Please consider the disagreement that you and your partner had in the past month that you found the most troublesome. If you are not currently in a relationship, please use an example from your most relationship. Please describe below:

How much control did you feel that you had over the resolution to this disagreement?

None at all

$\begin{array}{lllllll}1 & 2 & 3 & 4 & 5 & 6 & 7 \\ \text { all } & & & & & & \text { Total control }\end{array}$

Was resolving this disagreement important to you?
1
2 .
3
4
56
Extremely

None at all

Did this disagreement only affect you in a minor way, or do you feel that it affected almost everything you did?

$\begin{array}{ccccccc}1 & 2 & 3 & 4 & 5 & 6 & 7 \\ \text { Hardly at all } & & & & & & \text { Almost everything }\end{array}$

Which of the following strategies do you think you would find yourself using in response to this situation?

Never

1. accepted that there was nothing you could do to change your situation?

2. tried to just take whatever came your way?

3. talked with friends or relatives about your problems?

4. tried to do things which you typically enjoy?

5. sought out information that would help you resolve your problems?

6. blamed others for creating your problems or making them worse?
Seldom Sometimes $\begin{gathered}\text { Often Almost } \\ \text { always }\end{gathered}$
alwa

$\begin{array}{lllll}0 & 1 & 2 & 3 & 4 \\ 0 & 1 & 2 & 3 & 4 \\ 0 & 1 & 2 & 3 & 4 \\ 0 & 1 & 2 & 3 & 4 \\ 0 & 1 & 2 & 3 & 4 \\ 0 & 1 & 2 & 3 & 4\end{array}$


7. sought the advice of others to resolve your problems? 0

8. blamed yourself for your problems?

9. exercised? 0

10. fantasized or thought about unreal things (e.g., the perfect 0 revenge, or winning a million dollars) to feel better?

11. been very emotional compared to your usual self?

12. gone over your problems in your mind over and over again?

13. asked others for help?

14. thought about your problems a lot?

15 . became involved in recreation or pleasure activities?

16. worried about your problems a lot?

17. tried to keep your mind off things that are upsetting you?

18. tried to distract yourself from your troubles?

19. avoided thinking about your problems?

20 . made plans to overcome your problems?

21. told jokes about your situation?

22. thought a lot about who is responsible for your problems (besides yourself)?

23. shared humorous stories etc. to cheer yourself and others up?

24. told yourself that other people have dealt with problems such as yours?

25. thought a lot about how you have brought your problems on yourself?

26. decided to wait and see how things turn out?

27 . wished the situation would go away or be over with?

28. decided that your current problems are a result of your own past actions?

29. gone shopping?

30. asserted yourself and taken positive action on problems that are getting you down?

31. sought reassurance and moral support from others?

32. resigned yourself to your problems?

33. thought about how your problems have been caused by other people?

34. daydreamed about how things may turn out?

0

0

0

0

0

0

0

\section{1}

\section{1}

$$
1
$$

$$
1
$$

2

3

2

3

2

3

2

3

2

$\begin{array}{ll}3 & 4 \\ 3 & 4\end{array}$

0

2

1

$2 \quad 3 \quad 4$

0

.

$\begin{array}{lllll}1 & 2 & 3 & 4\end{array}$

$\begin{array}{lllll}0 & 1 & 2 & 3 & 4 \\ 0 & 1 & 2 & 3 & 4\end{array}$

$\begin{array}{lllll}1 & 2 & 3 & 4 \\ 0 & 1 & 2 & 3 & 4 \\ 0 & 1 & 2 & 3 & 4\end{array}$

$\begin{array}{lllll}1 & 2 & 3 & 4\end{array}$


35. been very emotional in how you react, even to little things?

36. decided that you can grow and learn through your problems?

37. told yourself that other people have problems like your own?

38. wished I was a stronger person or better at dealing with problems?

39. looked for how you can learn something out of your bad situation?

40. asked for God's guidance?

41. kept your feelings bottled up inside?

42. found yourself crying more than usual?

43. tried to act as if you were not upset?

44. prayed for help?

45. gone out?

46. held in your feelings?

47. tried to act as if you weren't feeling bad?

48. taken steps to overcome your problems?

49. made humorous comments or wise cracks?

50. told others that you were depressed or emotionally upset?

$\begin{array}{lllll}0 & 1 & 2 & 3 & 4 \\ 0 & 1 & 2 & 3 & 4 \\ 0 & 1 & 2 & 3 & 4 \\ 0 & 1 & 2 & 3 & 4 \\ 0 & 1 & 2 & 3 & 4 \\ 0 & 1 & 2 & 3 & 4 \\ 0 & 1 & 2 & 3 & 4 \\ 0 & 1 & 2 & 3 & 4 \\ 0 & 1 & 2 & 3 & 4 \\ 0 & 1 & 2 & 3 & 4 \\ 0 & 1 & 2 & 3 & 4 \\ 0 & 1 & 2 & 3 & 4 \\ 0 & 1 & 2 & 3 & 4 \\ 0 & 1 & 2 & 3 & 4 \\ 0 & 1 & 2 & 3 & 4 \\ 0 & 1 & 2 & 3 & 4\end{array}$




\section{LIFE EVENTS QUESTIONNAIRE (Study 1)}

The purpose of this questionnaire is to identify significant life experiences in one's life. The events listed below are far more common than many people realize. Please read each question carefully and circle the answers that best describe your experience.

1. Have you ever experienced a natural disaster (a flood, hurricane, earthquake, etc.)?

never once twice 3 times 4 times 5 times more than 5 times

If this happened:

When did it happen?

In the past year; 10-15 years ago; 2-5 years ago; 6-10 years ago

Did you experience fear, helplessness, or horror at what happened? yes / no

Were you seriously injured? yes / no

Was someone you cared about or close by seriously injured or killed? yes / no

Did you think you or a loved one was in danger of being killed by the disaster? yes / no

2. Were you involved in a motor vehicle accident for which you received medical attention or that badly injured or killed someone?

never once twice 3 times 4 times 5 times more than 5 times

If this happened:

When did it happen?

In the past year; 2-5 years ago; 6-10 years ago 10-15 years ago; when you were less than 5 years old

Did you experience fear, helplessness, or horror when it happened? yes / no

Were you seriously injured? yes / no

3. Have you been involved in any other kind of accident where you or someone else was badly hurt? (examples: a plane crash, a drowning or near drowning, an electrical or machinery accident, an explosion, home fire, chemical leak, or overexposure to radiation or toxic chemicals)

never once twice 3 times 4 times 5 times more than 5 times

If this happened:

When did it happen?

In the past year; 2-5 years ago; 6-10 years ago 10-15 years ago; when you were less than 5 years old

Did you experience fear, helplessness, or horror when it happened? yes / no

Were you seriously injured? yes / no

4. Have you lived, worked, or had military service in a war zone? yes / no If yes, were you ever exposed to warfare or combat? (for example: in the vicinity of a rocket attack or people being fired upon; seeing someone getting wounded or killed)

\section{never once twice 3 times 4 times 5 times more than 5 times}

If this happened: 
When did it happen?

In the past year;

Did you experience fear, helplessness, or horror when it happened? yes / no

2-5 years ago;

6-10 years ago

Were you seriously injured? yes / no

5. Have you experienced the unexpected and sudden death of a close friend or loved one?

never once twice 3 times 4 times 5 times more than 5 times

If this happened:

When did it happen?

In the past year; 10-15 years ago; 2-5 years ago; 6-10 years ago

Did you experience fear, helplessness, or horror when it happened? yes / no

Were you seriously injured? yes / no

6. Has a loved one (who is living) ever experienced a life threatening or permanently disabling accident, assault, or illness? (examples: spinal cord injury, rape, life threatening virus)

never once twice 3 times 4 times 5 times more than 5 times

If this happened:

When did it happen? In the past year; 10-15 years ago; 2-5 years ago; 6-10 years ago Did you experience fear, helplessness, or horror when it happened? yes / no

7. Have you ever had a life threatening illness?

never once twice 3 times 4 times 5 times more than 5 times

If this happened:

When did it happen?

In the past year; 2-5 years ago; 6-10 years ago 10-15 years ago; when you were less than 5 years old

Did you experience fear, helplessness, or horror when it happened? yes / no

8. Have you been robbed or been present during a robbery - where the robber(s) used or displayed a weapon?

never once twice 3 times 4 times 5 times more than 5 times

If this happened:

When did it happen?

In the past year; 2-5 years ago; 6-10 years ago 10-15 years ago; when you were less than 5 years old

Did you experience fear, helplessness, or horror when it happened? yes / no

Were you seriously injured? yes / no

9. Have you ever been hit or beaten up and badly hurt by a stranger or someone you didn't know very well? 
never once twice 3 times 4 times 5 times more than 5 times

If this happened:

When did it happen?

In the past year;

2-5 years ago; 6-10 years ago

Did you experience fear, helplessness, or horror when it happened? yes / no

Were you seriously injured? yes / no

10. Have you seen a stranger (or someone you didn't know very well) attack or beat up another someone and seriously injure or kill them?

never once twice 3 times 4 times 5 times more than 5 times

If this happened:

When did it happen?

In the past year; 10-15 years ago; 2-5 years ago; when you were less than 5 years old

Did you experience fear, helplessness, or horror when it happened? yes / no

11. Has anyone threatened to kill you or cause you serious physical harm?

never once twice 3 times 4 times 5 times more than 5 times

If this happened:

When did it happen?

In the past year; 10-15 years ago; 2-5 years ago; 6-10 years ago

Did you experience fear, helplessness, or horror when it happened? yes / no

Was this person a stranger? yes / no friend or acquaintance? yes / no relative? yes / no intimate partner? yes / no

12. While growing up, were you physically punished in a way that resulted in bruises, burns, cuts, or broken bones?

never once twice 3 times 4 times 5 times more than 5 times

If this happened:

Did you experience fear, helplessness, or horror when it happened? yes / no

13. While growing up, did you see or hear family violence? (such as your father hitting your mother; or any family member beating up or inflicting bruises, bruises, or cuts on another family member)

never once twice 3 times 4 times 5 times more than 5 times

If this happened:

Did you experience fear, helplessness, or horror when it happened? yes / no

14. Have you ever been slapped, punched, kicked, beaten up, or otherwise physically hurt by your spouse (or former spouse), a boyfriend/girlfriend, or some other intimate partner? 
never once twice 3 times 4 times 5 times more than 5 times

If this happened:

When did it happen?

In the past year;

2-5 years ago;

6-10 years ago

Did you experience fear, helplessness, or horror when it happened? yes / no

Were you seriously injured? yes / no

Has more than one intimate partner physically hurt you? yes / no

If yes, how many have hurt you?

15. Before your $13^{\text {th }}$ birthday: Did anyone - who was at least 5 years older than you - touch or fondle your body in a sexual way or make you touch or fondle their body in a sexual way?

never once twice 3 times 4 times 5 times more than 5 times

If this happened:

Did you experience fear, helplessness, or horror when it happened? yes / no

Were you seriously injured? yes / no

Was the person a stranger? yes / no parent or caregiver? yes / no

friend or acquaintance? yes / no other relative? yes / no

Was threat or force used? yes / no

Was there oral, anal, or vaginal penetration? yes / no

16. Before your $13^{\text {th }}$ birthday: Did anyone close to your age touch sexual parts of your body or make you touch sexual parts of their body -against your will or without your consent?

never once twice 3 times 4 times 5 times more than 5 times

If this happened:

Did you experience fear, helplessness, or horror when it happened? yes / no

Were you seriously injured? yes / no

Was the person a stranger? yes / no parent or caregiver? yes / no friend or acquaintance? yes / no other relative? yes / no

Was threat or force used? yes / no

Was there oral, anal, or vaginal penetration? yes / no

17. After your $13^{\text {th }}$ birthday and before your $18^{\text {th }}$ birthday: Did anyone touch sexual parts of your body or made you touch sexual parts of their body - against your will or without your consent?

never once twice 3 times 4 times 5 times more than 5 times

If this happened:

Did you experience fear, helplessness, or horror when it happened? yes / no

Were you seriously injured? yes / no

Was the person a stranger? yes / no parent or caregiver? yes / no

friend or acquaintance? yes / no other relative? yes / no

Was threat or force used? yes / no

Was there oral, anal, or vaginal penetration? yes / no 
18. After your $18^{\text {th }}$ birthday: Did anyone touch sexual parts of your body or made you touch sexual parts of their body - against your will or without your consent?

never once twice 3 times 4 times 5 times more than 5 times

If this happened:

Did you experience fear, helplessness, or horror when it happened? yes / no

Were you seriously injured? yes / no

Was the person a stranger? yes / no parent or caregiver? yes / no

friend or acquaintance? yes / no

Was threat or force used? yes / no

other relative? yes / no

Was there oral, anal, or vaginal penetration? yes / no

19. Has anyone stalked you - in other words: followed you or kept track of your activities causing you to feel intimidated or concerned for your safety?

never once twice 3 times 4 times 5 times more than 5 times

If this happened:

When did it happen? In the past year; 2-5 years ago; 6-10 years ago

Was the person a stranger? yes / no when you were less than 5 years old friend or acquaintance? yes / no other relative? yes / no

Did you experience fear, helplessness, or horror when it happened? yes / no

20. Have you ever had a miscarriage?

never once twice 3 times 4 times 5 times more than 5 times

If this happened:

When did it happen? ___ In the past year; 2-5 years ago;

Did you experience fear, helplessness, or horror when it happened? yes / no

Were you seriously injured? yes / no

21. Have you ever had an abortion?

never once twice 3 times 4 times 5 times more than 5 times

If this happened:

When did it happen?

In the past year;

2-5 years ago;

6-10 years ago

Did you experience fear, helplessness, or horror when it happened? yes / no

22. Have you ever had something happened to you that you believe represented an experience of discrimination (e.g., religious, racial, sex)?

never once twice 3 times 4 times 5 times more than 5 times

If this happened: 
When did it happen? In the past year; 10-15 years ago; 2-5 years ago; 6-10 years ago when you were less than 5 years old
Was the source of the discrimination a
stranger? yes / no
friend or acquaintance? yes / no
someone in your workplace/school? yes / no
an organization/institution yes / no

Did you experience fear, helplessness, or horror when it happened? yes / no

23. Have you experienced (or seen) any other events that were life threatening, caused serious injury, or were highly disturbing and distressing? (examples: lost in the wilderness; a serious animal bite; violent death of a pet; being kidnapped and held hostage; seeing a mutilated body or parts)

never once twice 3 times 4 times 5 times more than 5 times

Please describe:

If this happened:

Did you experience fear, helplessness, or horror when it happened? yes / no

Were you seriously injured? yes / no

24. If any of the events (listed above) happened to you, which one event

CAUSES YOU THE MOST DISTRESS?

Indicate Item \#:

When did this event (last) happen (your age or date)?

How much distress (anxiety, worry, sadness, or grief) does this event cause you?

\begin{tabular}{|c|c|c|c|c|}
\hline $\begin{array}{c}\text { None } \\
\text { happened }\end{array}$ & $\begin{array}{c}\text { no } \\
\text { distress }\end{array}$ & $\begin{array}{c}\text { slight } \\
\text { distress }\end{array}$ & $\begin{array}{c}\text { moderate } \\
\text { distress }\end{array}$ & $\begin{array}{c}\text { considerable } \\
\text { distress }\end{array}$ \\
\hline
\end{tabular}




\section{LIFE EVENTS QUESTIONNAIRE - revised (Study 2)}

The purpose of this questionnaire is to identify significant life experiences in one's life. The events listed below are far more common than many people realize. Please read each question carefully and circle the answers that best describe your experience.

1. Have you ever experienced a natural disaster (a flood, hurricane, earthquake, etc.)?

never once twice 3 times 4 times 5 times more than 5 times

If this happened:

When did it happen?

In the past year; 10-15 years ago; 2-5 years ago; 6-10 years ago

Did you experience fear, helplessness, or horror at what happened? yes / no

Were you seriously injured? yes / no

Was someone you cared about or close by seriously injured or killed? yes / no

Did you think you or a loved one was in danger of being killed by the disaster? yes / no

2. Were you involved in a motor vehicle accident for which you received medical attention or that badly injured or killed someone?

never once twice 3 times 4 times 5 times more than 5 times

If this happened:

When did it happen?

In the past year;

2-5 years ago; 6-10 years ago

10-15 years ago; when you were less than 5 years old

Did you experience fear, helplessness, or horror when it happened? yes / no

Were you seriously injured? yes / no

3. Have you been involved in any other kind of accident where you or someone else was badly hurt? (examples: a plane crash, a drowning or near drowning, an electrical or machinery accident, an explosion, home fire, chemical leak, or overexposure to radiation or toxic chemicals)

never once twice 3 times 4 times 5 times more than 5 times

If this happened:

When did it happen?

In the past year;

10-15 years ago;

2-5 years ago;

6-10 years ago when you were less than 5 years old

Did you experience fear, helplessness, or horror when it happened? yes / no

Were you seriously injured? yes / no

4. Have you lived, worked, or had military service in a war zone? yes / no

If yes, were you ever exposed to warfare or combat? (for example: in the vicinity of a rocket attack or people being fired upon; seeing someone getting wounded or killed)?

never once twice 3 times 4 times 5 times more than 5 times 
If this happened:

When did it happen?

In the past year;

2-5 years ago; 6-10 years ago 10-15 years ago; when you were less than 5 years old

Did you experience fear, helplessness, or horror when it happened? yes / no

Were you seriously injured? yes / no

4. Have you experienced the unexpected and sudden death of a close friend or loved one?

never once twice 3 times 4 times 5 times more than 5 times

If this happened:

When did it happen?

In the past year; 2-5 years ago; 6-10 years ago 10-15 years ago; when you were less than 5 years old

Did you experience fear, helplessness, or horror when it happened? yes / no

Were you seriously injured? yes / no

5. Has a loved one (who is living) ever experienced a life threatening or permanently disabling accident, assault, or illness? (examples: spinal cord injury, rape, life threatening virus)

never once twice 3 times 4 times 5 times more than 5 times

If this happened:

When did it happen? In the past year; 10-15 years ago; 2-5 years ago; 6-10 years ago

Did you experience fear, helplessness, or horror when it happened? yes / no

6. Have you ever had a life threatening illness?

never once twice 3 times 4 times 5 times more than 5 times

If this happened:

When did it happen?

In the past year;

2-5 years ago; 6-10 years ago 10-15 years ago; when you were less than 5 years old

Did you experience fear, helplessness, or horror when it happened? yes / no

8. Have you been robbed or been present during a robbery - where the robber(s) used or displayed a weapon?

never once twice 3 times 4 times 5 times more than 5 times

If this happened:

When did it happen?

In the past year; 2-5 years ago; 6-10 years ago 10-15 years ago; when you were less than 5 years old

Did you experience fear, helplessness, or horror when it happened? yes / no

Were you seriously injured? yes / no

9. Have you ever been hit or beaten up and badly hurt by a stranger or someone you didn't know very well? 
never once twice 3 times 4 times 5 times more than 5 times

If this happened:

When did it happen?

In the past year;

2-5 years ago;

6-10 years ago

Did you experience fear, helplessness, or horror when it happened? yes / no

Were you seriously injured? yes / no

78. Have you seen a stranger (or someone you didn't know very well) attack or beat up another someone and seriously injure or kill them?

never once twice 3 times 4 times 5 times more than 5 times

If this happened:

When did it happen?

In the past year; 2-5 years ago; 6-10 years ago 10-15 years ago; when you were less than 5 years old

Did you experience fear, helplessness, or horror when it happened? yes / no

79. Has anyone threatened to kill you or cause you serious physical harm?

never once twice 3 times 4 times 5 times more than 5 times

If this happened:

When did it happen?

In the past year; 10-15 years ago; 2-5 years ago; when you were less than 5 years old

Did you experience fear, helplessness, or horror when it happened? yes / no

Was this person a stranger? yes / no

friend or acquaintance? yes / no

a boyfriend? yes / no

relative? yes / no intimate partner? yes / no

80. While growing up, were you physically punished in a way that resulted in bruises, burns, cuts, or broken bones?

never once twice 3 times 4 times 5 times more than 5 times

If this happened:

Did you experience fear, helplessness, or horror when it happened? yes / no

81. While growing up, did you see or hear family violence? (such as your father hitting your mother; or any family member beating up or inflicting bruises, bruises, or cuts on another family member)

never once twice 3 times 4 times 5 times more than 5 times

If this happened:

Did you experience fear, helplessness, or horror when it happened? yes / no 
82. Have you ever been slapped, punched, kicked, beaten up, or otherwise physically hurt by your spouse (or former spouse), a boyfriend, or some other intimate partner?

never once twice 3 times 4 times 5 times more than 5 times

If this happened:

When did it happen?

In the past year; 2-5 years ago; 6-10 years ago

Did this happen in your current relationship or past relationship?

Did you experience fear, helplessness, or horror when it happened? yes / no

Were you seriously injured? yes / no

Has more than one intimate partner physically hurt you? yes / no

If yes, how many have hurt you?

15. Have you ever been ridiculed, insulted or humiliated without reason by your spouse (or former spouse), a boyfriend, or some other intimate partner?

never once twice 3 times 4 times 5 times more than 5 times

If this happened:

When did it happen?

In the past year;

2-5 years ago;

6-10 years ago

Did this happen in your current relationship or past relationship?

Did you experience fear, helplessness, or horror when it happened? yes / no

Has more than one intimate partner emotionally/psychologically hurt you? yes / no

If yes, how many have hurt you?

16. Before your $13^{\text {th }}$ birthday: Did anyone - who was at least 5 years older than you - touch or fondle your body in a sexual way or make you touch or fondle their body in a sexual way?

never once twice 3 times 4 times 5 times more than 5 times

If this happened:

Did you experience fear, helplessness, or horror when it happened? yes / no

Were you seriously injured? yes / no

Was the person a stranger? yes / no parent or caregiver? yes / no

friend or acquaintance? yes / no other relative? yes / no

Was threat or force used? yes / no

Was there oral, anal, or vaginal penetration? yes / no

17. Before your $13^{\text {th }}$ birthday: Did anyone close to your age touch sexual parts of your body or make you touch sexual parts of their body -against your will or without your consent?

never once twice 3 times 4 times 5 times more than 5 times

If this happened:

Did you experience fear, helplessness, or horror when it happened? yes / no

Were you seriously injured? yes / no

Was the person a stranger? yes / no friend or acquaintance? yes / no 
parent or caregiver? yes / no other relative? yes / no

Was threat or force used? yes / no

Was there oral, anal, or vaginal penetration? yes / no

18. After your $13^{\text {th }}$ birthday and before your $18^{\text {th }}$ birthday: Did anyone touch sexual parts of your body or made you touch sexual parts of their body - against your will or without your consent?

never once twice 3 times 4 times 5 times more than 5 times

If this happened:

Did you experience fear, helplessness, or horror when it happened? yes / no

Were you seriously injured? yes / no

Was the person

a stranger? yes / no friend or acquaintance? yes / no

a boyfriend? yes / no parent or caregiver? yes / no other relative? yes / no

If a boyfriend, is this your

Was threat or force used?

current partner or

previous partner?

Was there oral, anal, or vaginal penetration? yes / no

19. After your $18^{\text {th }}$ birthday: Did anyone touch sexual parts of your body or made you touch sexual parts of their body - against your will or without your consent?

never once twice 3 times 4 times 5 times more than 5 times

If this happened:

Did you experience fear, helplessness, or horror when it happened? yes / no

Were you seriously injured? yes / no

Was the person

a stranger? yes / no friend or acquaintance? yes / no

a boyfriend? yes / no parent or caregiver? yes / no other relative? yes / no

If a boyfriend, is this your ___ current partner or __ previous partner?

Was threat or force used? yes / no

Was there oral, anal, or vaginal penetration? yes / no

20. Has anyone stalked you - in other words: followed you or kept track of your activities causing you to feel intimidated or concerned for your safety?

never once twice 3 times 4 times 5 times more than 5 times

If this happened:

When did it happen?

In the past year; 10-15 years ago; 2-5 years ago; 6-10 years ago

Was the person 
If a boyfriend, is this your ___ current partner or ___ previous partner? Did you experience fear, helplessness, or horror when it happened? yes / no

21. Have you ever had a miscarriage?

never once twice 3 times 4 times 5 times more than 5 times

If this happened:

When did it happen?

In the past year;

2-5 years ago;

6-10 years ago

Did you experience fear, helplessness, or horror when it happened? yes / no

Were you seriously injured? yes / no

22. Have you ever had an abortion?

never once twice 3 times 4 times 5 times more than 5 times

If this happened:

When did it happen?

In the past year;

2-5 years ago;

6-10 years ago

Did you experience fear, helplessness, or horror when it happened? yes / no

23. Have you ever had something happened to you that you believe represented an experience of discrimination (e.g., religious, racial, sex)?

never once twice 3 times 4 times 5 times more than 5 times

If this happened:

When did it happen?

In the past year; 10-15 years ago; 2-5 years ago; 6-10 years ago

Was the source of the discrimination a stranger? yes / no when you were less than 5 years old

friend or acquaintance? yes / no

someone in your workplace/school? yes / no

an organization/institution yes / no

Did you experience fear, helplessness, or horror when it happened? yes / no

24. Have you experienced (or seen) any other events that were life threatening, caused serious injury, or were highly disturbing and distressing? (examples: lost in the wilderness; a serious animal bite; violent death of a pet; being kidnapped and held hostage; seeing a mutilated body or parts)?

never once twice 3 times 4 times 5 times more than 5 times

Please describe: 
If this happened:

Did you experience fear, helplessness, or horror when it happened? yes / no Were you seriously injured? yes / no

25. If any of the events (listed above) happened to you, which one event CAUSES YOU THE MOST DISTRESS?

Indicate Item \#:

When did this event (last) happen (your age or date)?

How much distress (anxiety, worry, sadness, or grief) does this event cause you?

\begin{tabular}{cccccc}
$\begin{array}{c}\text { None } \\
\text { happened }\end{array}$ & $\begin{array}{c}\text { no } \\
\text { distress }\end{array}$ & $\begin{array}{c}\text { slight } \\
\text { distress }\end{array}$ & $\begin{array}{c}\text { moderate } \\
\text { distress }\end{array}$ & $\begin{array}{c}\text { considerable } \\
\text { distress }\end{array}$ & $\begin{array}{c}\text { extreme } \\
\text { distress }\end{array}$ \\
\hline
\end{tabular}




\section{SOCIAL PROVISIONS}

In answering the next set of questions, please think about your current relationships with your friends. If you feel a question accurately describes your relationships with your friends, you would say "yes". If the question does not describe your relationship, you would say "no". If you cannot decide whether the question describes your relationships with your friends, you may say "not sure".

\section{No Not sure Yes}

1. Are there friends you can depend on to help you, if you really need it? $\quad 1 \quad 2$

2. Do you feel you could not turn to your friends for guidance in times of stress?

3. Are there friends who enjoy the same social activities that you do? 1

4. Do you feel personally responsible for the well-being of your friends? 1

5. Do you feel your friends do not respect your skills and abilities? $\quad 1$

23

6. If something went wrong, do you feel that none of your friends would 1 come to your assistance?

7. Do your relationships with your friends provide you with a sense of 1 emotional security and well being?

8. Do you feel your competence and skill are recognized by your friends? 1

9. Do you feel none of your friends share your interests and concerns?

10. Do you feel none of your friends really rely on you for their well-being?

11. Is there a trustworthy friend you could turn to for advice, if you were having problems?

12. Do you feel you lack emotional closeness with your friends? 
In answering the next set of questions, please think about your current relationships with your parents.

\section{No Not sure Yes}

1. Can you depend on your parents to help you, if you really need it? $\quad \begin{array}{llll}2 & 2\end{array}$

2. Do you feel you could not turn to your parents for guidance in times $\begin{array}{lll}1 & 2\end{array}$ of stress?

3. Do your parents enjoy the same social activities that you do? $\quad 1 \quad 2 \quad 3$

4. Do you feel personally responsible for the well-being of your parents? $1 \quad 2 \quad 3$

5. Do you feel your parents do not respect your skills and abilities? $\quad 1 \quad \begin{array}{lll}2 & 3\end{array}$

6. If something went wrong, do you feel that your parents would $\quad \begin{array}{llll}1 & 2 & 3\end{array}$ not come to your assistance?

7. Does your relationship with your parents provide you with $\quad \begin{array}{llll}1 & 2 & 3\end{array}$ a sense of emotional security and well-being?

8. Do you feel your competence and skill are recognized by your parents? $1 \quad 2 \quad 3$

9. Do you feel your parents do not share your interests and concerns? $\quad \begin{array}{llll}1 & 2 & 3\end{array}$

10. Do you feel your parents do not really rely on you for their $\quad 1 \quad 243$ well-being?

11. Could you turn to your parents for advise, if you were having $\quad \begin{array}{llll}1 & 2 & 3\end{array}$ problems?

12. Do you feel you lack emotional closeness with your parents? $\quad \begin{array}{lll}1 & 2 & 3\end{array}$ 
In answering the next set of questions, please think about your current relationships with your partner.

\section{No Not sure Yes}

1. Can you depend on your partner to help you, if you really need it? $\quad 1 \quad \begin{array}{lll}1 & 2 & 3\end{array}$

2. Do you feel you could not turn to your partner for guidance in times $\quad \begin{array}{lll}1 & 2\end{array}$ of stress?

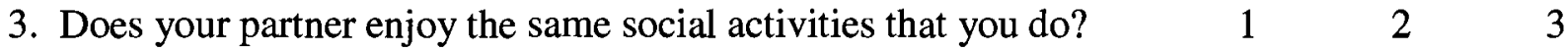

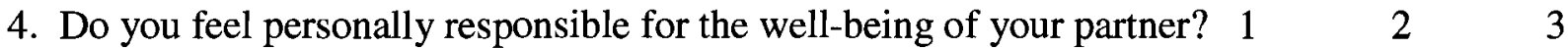

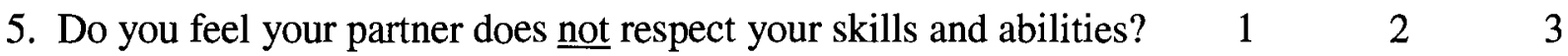

6. If something went wrong, do you feel that your partner would not $\quad \begin{array}{llll}1 & 2 & 3\end{array}$ come to your assistance?

7. Does your relationship with your partner provide you with $\quad 1 \quad 3 \quad 2 \quad 3$ a sense of emotional security and well-being?

8. Do you feel your competence and skill are recognized by your partner? $1 \quad 2 \quad 3$

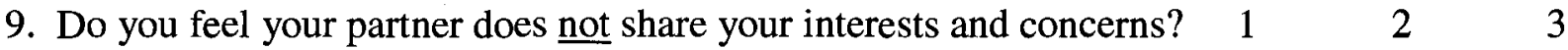

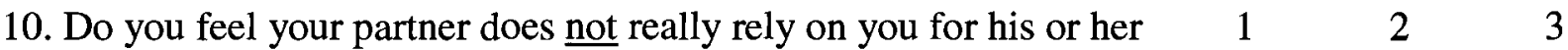
well-being?

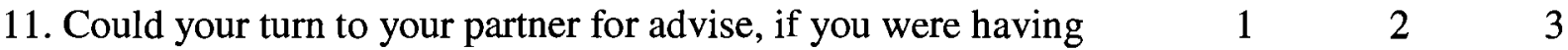
problems?

12. Do you feel you lack emotional closeness with your partner? $\quad 1 \quad 2$ 


\section{BECK INVENTORY}

On this questionnaire are groups of statements. Please read the entire group of statements of each category. Then pick out ONE statement in that group which best describes the way you feel. Check off the number beside the statement you have chosen.

1. 0 = I do not feel sad

$1=$ I feel sad or blue

$2 \mathrm{a}=\mathrm{I}$ am blue or sad all of the time and I can't snap out of it

$2 b=I$ am so sad or unhappy that it is very painful

3 = I am so sad or unhappy that I can't stand it

2. $-0=I$ am not particularly pessimistic or discouraged about the future

$1=I$ feel discouraged about the future

$2 \mathrm{a}=\mathrm{I}$ feel I have nothing to look forward to

$2 \mathrm{~b}=\mathrm{I}$ feel I won't every get over my troubles

$3=\mathrm{I}$ feel that the future is hopeless and things cannot improve

3. $\_$_ 0 I do not feel like a failure

$1=\mathrm{I}$ feel $\mathrm{I}$ have failed more than the average person

$2 \mathrm{a}=\mathrm{I}$ feel I have accomplished very little that is worthwhile or that means anything

$2 b=$ As I look back on my life, all I can see is a lot of failures

$3=$ I feel I am a complete failure as a person

4. $\quad 0=\mathrm{I}$ am not particularly dissatisfied

$1 \mathrm{a}=\mathrm{I}$ feel bored most of the time

$1 \mathrm{~b}=\mathrm{I}$ don't enjoy things the way I used to

$2=I$ don't get satisfaction out of anything anymore

$3=\mathrm{I}$ am dissatisfied with everything

5. __ 0 = I don't feel particularly guilty

$1=I$ feel bad or unworthy a good part of the time

$2 \mathrm{a}=\mathrm{I}$ feel quite guilty

$2 b=I$ feel bad or unworthy practically of the time now

$3=I$ feel as though I am very bad or worthless

6. $\quad 0$ _ $\quad 0$ I don't feel I am being punished

1 - I I have a feeling that something bad may happen to me

$2=$ I feel I am being punished or will be punished

$3 \mathrm{a}=\mathrm{I}$ feel I deserve to be punished

$3 b=I$ want to be punished

7. 0 _ 0 I don't feel disappointed in myself

$1 \mathrm{a}=\mathrm{I}$ am disappointed in myself

$1 b=$ I don't like myself 
$2=\mathrm{I}$ am disgusted with myself

3 = I hate myself

8. $0=$ I do not feel I am any worse than anybody else

- 1 = I am very critical of myself for my weaknesses or mistakes

_ $2 \mathrm{a}=$ I blame myself for everything that goes wrong

$2 \mathrm{~b}=\mathrm{I}$ feel I have many bad faults

9. $0=\mathrm{I}$ don't have thoughts of harming myself

- 1 = I have thoughts of harming myself but I would not carry them out

$2 \mathrm{a}=\mathrm{I}$ feel I would be better off dead

— $2 \mathrm{~b}=\mathrm{I}$ have definite plans about committing suicide

$2 c=I$ feel my family would be better off if I were dead

3 = I would kill myself if I could

10.

$0=I$ don't cry anymore than usual

$1=\mathrm{I}$ cry more now than I used to

2 = I cry all the time now. I can't stop it

- $3=$ I used to be able to cry but now I can't cry at all even though I want to

11. $0=\mathrm{I}$ am no more irritated now than I ever am

— $1=\mathrm{I}$ get annoyed or irritated more easily than I used to

$2=$ I get irritated all the time

$3=\mathrm{I}$ don't get irritated at all the things that used to irritate me.

12. $0=\mathrm{I}$ have not lost interest in other people

- $1=\mathrm{I}$ am less interested in other people than I used to be

2 = I have lost most of my interest in other people and I have little feeling for them

3 = I have lost all my interest in other people and don't care about them at all

13. _ $0=$ I make decisions about as well as ever

— $1=\mathrm{I}$ am less sure of myself now and try to put off making decisions

- 2 = I can't make decisions anymore without help

_ 3 = I can't make decisions at all anymore

14. $0=\mathrm{I}$ don't feel I look any worse than I used to

$1=\mathrm{I}$ am worried that $\mathrm{I}$ am looking old or unattractive

${ }_{2} 2=\mathrm{I}$ feel that there permanent changes in my appearance and they make me look unattractive

_ 3 = I feel that I am ugly or repulsive looking

15. _ $0=\mathrm{I}$ can work about as well as before

$1 \mathrm{a}=$ It takes extra effort to get started at doing something

$1 \mathrm{~b}=\mathrm{I}$ don't work as well as I used to

2 = I have to push myself very hard to do anything

3 = I can't do any work at all 
16. $0=$ I can sleep as well as usual

$1=\mathrm{I}$ wake up more tired in the morning than I used to

2 = I wake up 1-2 hours earlier than usual and find it hard to get back to sleep

3 = I wake up early every day and can't get more than 5 hours sleep

17. $0=\mathrm{I}$ don't get anymore tired than usual

$1=$ I get tired more easily than I used to

2 = I get tired from doing anything

3 = I get too tired to do anything

18. _ $0=$ My appetite is no worse than usual

- $1=$ My appetite is not as good as it used to be

2 = My appetite is much worse now

- $3=$ I have no appetite at all any more

19. _ $0=$ I haven't lost much weight, if any, lately

$1=$ I have lost more than 5 pounds

— 2 = I have lost more than 10 pounds

3 = I have lost more than 15 pounds

20. _ $0=\mathrm{I}$ am no more concerned about my health than usual

$1=\mathrm{I}$ am concerned about aches and pains or upset stomach or constipation or other unpleasant feelings in my body

$2=\mathrm{I}$ am so concerned with how I feel or what I feel that it's hard to think of much else

_ 3 = I am completely absorbed in what I feel

21. _ $0=\mathrm{I}$ have not noticed any recent change in my interest in sex

$1=\mathrm{I}$ am less interested in sex than I used to be

$2=\mathrm{I}$ am much less interested in sex now

$3=$ I have lost interest in sex completely 


\section{Picture questions (Study 1)}

1. Describe, in a couple of lines, what you see in the picture you just viewed.

2. On a scale of 0 to 7 , indicate how positive you consider this picture to be.

$\begin{array}{lllllllc}0 & 1 & 2 & 3 & 4 & 5 & 6 & \begin{array}{c}7 \\ \text { Extremely } \\ \text { positive }\end{array} \\ \text { all positive } & & & & & & & \end{array}$

3. On a scale of 0 to 7 , indicate how negative you consider this picture to be.

$\begin{array}{llllllll}0 & 1 & 2 & 3 & 4 & 5 & 6 & 7\end{array}$

Not at

all negative

Extremely

negative

\section{Coping Strategies Scale}

Based on your own sense of what is going on in the picture, how would you deal with the situation.

If I was in this situation, I would...

\begin{tabular}{lllcc}
\multicolumn{1}{c}{$\begin{array}{l}0 \\
\text { Not at all } \\
\text { likely }\end{array}$} & 1 & 2 & 3 & $\begin{array}{l}4 \\
\text { Extremely } \\
\text { likely }\end{array}$
\end{tabular}

1. accept that there is nothing I could do to change my situation.

2. blame myself for my problems.

3. tell others that I was really upset.

4. ask others for help or advice.

5. spend a lot of time thinking about my problems.

6. take time for recreation or pleasure activities.

7. make plans to overcome my concerns or problems.

8. avoid thinking about my problems.

9. tell jokes about my situation.

10. think a lot about who was responsible for my problems (besides me).

11. worry about my problems a lot.

$\begin{array}{lllll}0 & 1 & 2 & 3 & 4\end{array}$

$\begin{array}{lllll}0 & 1 & 2 & 3 & 4\end{array}$

$\begin{array}{lllll}0 & 1 & 2 & 3 & 4\end{array}$

$\begin{array}{lllll}0 & 1 & 2 & 3 & 4\end{array}$

$\begin{array}{lllll}0 & 1 & 2 & 3 & 4 \\ 0 & 1 & 2 & 3 & 4\end{array}$

$\begin{array}{lllll}0 & 1 & 2 & 3 & 4\end{array}$

$\begin{array}{lllll}0 & 1 & 2 & 3 & 4\end{array}$

12. make humorous comments or stories about my situation. $\begin{array}{llllll}0 & 1 & 2 & 3 & 4\end{array}$

13. wish the situation would just go away or be over with $\quad \begin{array}{llllll}0 & 1 & 2 & 3 & 4\end{array}$ 
14. think a lot about how I brought my problems on myself. $\begin{array}{llllll}0 & 1 & 2 & 3 & 4\end{array}$

$\begin{array}{llllll}\text { 15. decide to wait and see how things turned out. } & 0 & 1 & 2 & 3 & 4\end{array}$

16. try to keep my mind off things that were upsetting me. $\quad \begin{array}{llllll}0 & 1 & 2 & 3 & 4\end{array}$

17. seek reassurance and emotional support from others. $\quad \begin{array}{lllll}0 & 1 & 2 & 3 & 4\end{array}$

18. think about how my problems were

$\begin{array}{lllllll}\text { caused by other people. } & 0 & 1 & 2 & 3 & 4\end{array}$

19. cry, even if someone else was around. $\quad \begin{array}{lllll}0 & 1 & 2 & 3 & 4\end{array}$

20. look for how I could grow and learn through my situation. $\begin{array}{llllll}0 & 1 & 2 & 3 & 4\end{array}$

21. tell myself that other people have problems like mine. $\quad \begin{array}{llllll}0 & 1 & 2 & 3 & 4\end{array}$

22. do things to keep busy or active (e.g., exercised, went out). $\begin{array}{llllll}0 & 1 & 2 & 3 & 4\end{array}$

23. hold in my feelings. $\quad \begin{array}{lllll}0 & 1 & 2 & 3 & 4\end{array}$

24. daydream about how things may turn out. $\quad \begin{array}{llllll}0 & 1 & 2 & 3 & 4\end{array}$

25. try to act as if I wasn't feeling bad. $\quad \begin{array}{lllll}0 & 1 & 2 & 3 & 4\end{array}$

26. take steps to overcome the problem. $\quad \begin{array}{llllll}0 & 1 & 2 & 3 & 4\end{array}$

27. turn to God or my faith. $\quad \begin{array}{llllll}0 & 1 & 2 & 3 & 4\end{array}$ 


\section{APPRAISAL OF AMBIGUOUS SITUATIONS (Revised)}

The following is a list of situations that you might encounter at one time or another. Please imagine yourself in each situation, and then indicate how threatening, as well as how distressing you would find each of these events. We will also be asking you to indicate your thoughts concerning each of these situations. Please note that there are no right answers for each question - we are simply looking for your first reaction to each of these situations.

1) You and your partner are at a bar, and he sees you talking to a male friend. The next day, he does not return your calls.

a. How threatening would this situation be for you?

$\begin{array}{lllll}1 & 2 & 3 & 4 & 5\end{array}$

Not at all Extremely

b. How distressing would this situation be for you?

$\begin{array}{lllll}1 & 2 & 3 & 4 & 5 \\ \text { Not at all } & & & & \text { Extremely }\end{array}$

c. How much control do you think you would have over the outcome of this event?

$\begin{array}{lllll}1 & 2 & 3 & 4 & 5 \\ \text { None at all } & & & & \text { Complete control }\end{array}$

d. What would you be most likely to think in this situation?

He's probably just out with his friends and hasn't had a chance to call me back yet.

I'm not worried yet, I'll wait a while longer to see if he calls.

He's probably a little mad/jealous, but I'm sure he'll call back soon.

I'm a little worried that he is not going to call.

I should have known that speaking to that guy would make him angry, I am worried that he's going to break up with me.

2) You have a friend visiting from out of town and you inform your partner that you won't be able to spend much time with him for a couple of days. Your partner expresses concern that your friends are taking up a lot of your time, and that he hasn't been able to see you much lately.

a. How threatening would this situation be for you?

$\begin{array}{lllll}1 & 2 & 3 & 4 & 5 \\ \text { Not at all } & & & & \text { Extremely }\end{array}$

b. How distressing would this situation be for you?

$\begin{array}{lllll}1 & 2 & 3 & 4 & 5 \\ \text { Not at all } & & & & \text { Extremely }\end{array}$

c. How much control do you think you would have over the outcome of this event?

$\begin{array}{lllll}1 & 2 & 3 & 4 & 5 \\ \text { Not at all } & & & & \text { Complete control }\end{array}$

d. What would you be most likely to think in this situation? 
He really cares about me and just wants to spend as much time as possible together. He's just feeling left out.

It's too bad that we can't spend much time together lately, but I'm sure he'll understand.

I'm worried that if I don't devote more time to him, this may negatively affect our

relationship.

I'm concerned that if this keeps up, he may consider breaking up with me.

3) You're going to meet your partner's parents for the first time. Your partner tells you that he doesn't particularly like the outfit you are wearing to meet them, and suggests that you change.

\section{a. How threatening would this situation be for you?}

$\begin{array}{lllll}1 & 2 & 3 & 4 & 5\end{array}$

Not at all

5

Extremely

b. How distressing would this situation be for you?

$\begin{array}{lllll}1 & 2 & 3 & 4 & 5 \\ \text { Not at all } & & & & \text { Extremely }\end{array}$

c. How much control do you think you would have over the outcome of this event?

$\begin{array}{lllll}1 & 2 & 3 & 4 & 5 \\ \text { Not at all } & & & & \text { Complete control }\end{array}$

d. What would you most likely think in this situation?

He must really be concerned about his parents' opinions.

He's just nervous about the meeting and wants me to make a good first impression.

I appreciate his input but I'll just wear whatever makes me feel most comfortable.

I must not look very good in this outfit.

$\mathrm{He}$ is embarrassed to introduce me to his parents.

4) You are having a disagreement with your partner over some relationship issues and after stating your views on the situation, he gets frustrated and stomps out of the house.

a. How threatening would this situation be for you?

$\begin{array}{lllll}1 & 2 & 3 & 4 & 5 \\ \text { Not at all } & & & & \text { Extremely }\end{array}$

b. How distressing would this situation be for you?

$\begin{array}{lllll}1 & 2 & 3 & 4 & 5 \\ \text { Not at all } & & & & \text { Extremely }\end{array}$

c. How much control do you think you would have over the outcome of this event?

$\begin{array}{lllll}1 & 2 & 3 & 4 & 5 \\ \text { Not at all } & & & & \text { Complete control }\end{array}$

d. What would you be most likely to think in this situation?

His anger over this argument was kind of excessive.

Sometimes our arguments get out of hand but I'm sure things will be fine.

I'm not sure what to think... 
He's really mad at me, I'm worried about how things will be between us when he gets back. I shouldn't have made him so angry, I' $m$ sure he won't be back for a while.

5) You are out to dinner at a restaurant with your partner, and he tells you he thinks you need to cut down on the amount of food you are eating.

a. How threatening would this situation be for you?

$\begin{array}{lllll}1 & 2 & 3 & 4 & 5 \\ \text { Not at all } & & & & \text { Extremely }\end{array}$

b. How distressing would this situation be for you?

$\begin{array}{lllll}1 & 2 & 3 & 4 & 5 \\ \text { Not at all } & & & & \text { Extremely }\end{array}$

c. How much control do you think you would have over the outcome of this event?

$\begin{array}{lllll}1 & 2 & 3 & 4 & 5 \\ \text { Not at all } & & & & \text { Complete control }\end{array}$

d. What would you most likely think is the reason for his comment?

He is just looking out for my health, he really cares about me.

He probably just thinks that restaurant portions are too big these days, he didn't mean it as anything personal.

I'm not sure what to think...

He may be a little concerned that I will gain weight.

He thinks I am getting fat. 